\title{
Inverse zero-sum problems III
}

\author{
by \\ Weidong Gao (Tianjin), Alfred Geroldinger (Graz), \\ and David J. GRYNKIEWICZ (Graz)
}

1. Introduction. Let $G$ be a finite abelian group. The Davenport constant $\mathrm{D}(G)$ is the smallest integer $\ell \in \mathbb{N}$ such that every sequence $S$ over $G$ of length $|S| \geq \ell$ has a nontrivial zero-sum subsequence. This invariant has been studied since the 1960s, and it naturally occurs in various branches of combinatorics, number theory and geometry. Its precise value in terms of the group invariants is known for $p$-groups and for groups of rank at most two, among others. On the other hand, it is still unknown, for example, even for groups of the form $C_{n}^{3}$. The reader may want to consult one of the surveys [10, 13] for more information.

Inverse zero-sum problems ask for the structure of sequences that are extremal with respect to a certain property. Starting with the inverse problem for the Erdös-Ginzburg-Ziv constant in the 1980s, inverse zero-sum problems have attracted considerable attention in the last decade, partly motivated by applications to the theory of nonunique factorizations (see [14] and the two surveys mentioned above).

In the present paper, we study the inverse problem with respect to the Davenport constant. Thus we investigate the structure of minimal zero-sum sequences having length $\mathrm{D}(G)$. Cyclic groups, elementary 2-groups, $C_{2} \oplus C_{4}$ and $C_{3} \oplus C_{3}$ are groups having (up to automorphism) precisely one minimal zero-sum sequence of length $\mathrm{D}(G)$, and their structure is well-understood ([7, Section 5]). Let $S$ be a minimal zero-sum sequence of length $\mathrm{D}(G)$. For some very special types of groups, the structure of $S$ has been determined (see [8, 19, 20]). However, for general finite abelian groups, there is not even a conjecture on the structure of $S$, though the number of elements in $S$ whose order equals the exponent of the group has been investigated (see recent progress by Girard [15]). Here we concentrate on groups of the form

2010 Mathematics Subject Classification: 11P70, 11B50, 11B75.

Key words and phrases: Davenport constant, minimal zero-sum sequence, inverse zero-sum problem. 
$G=C_{n} \oplus C_{n}$ with $n \geq 2$. Then $\mathrm{D}(G)=2 n-1$, and the inverse problem with respect to $G$ was first studied in [7]. We say that $G$ has Property B if every minimal zero-sum sequence $S$ over $G$ of length $|S|=2 n-1$ contains an element with multiplicity $n-1$. It is easy to check that, if $G$ has Property B, then the structure of all minimal zero-sum sequences over $G$ is completely determined (see Lemma 2.3). The standing conjecture is that every group $G$ of the above form has Property $\mathbf{B}$, and this conjecture is supported by a variety of partial results (see [13, Section 5.2]). The main aim of the present paper is to show that Property $\mathbf{B}$ is multiplicative for groups of odd order.

Theorem. Let $G=C_{m n} \oplus C_{m n}$ with $m, n \in \mathbb{N}$ odd. If both $C_{m} \oplus C_{m}$ and $C_{n} \oplus C_{n}$ have Property $\mathbf{B}$, then $G$ has Property $\mathbf{B}$.

There is an earlier result of Gao and Geroldinger [9] stating that, if $n \in \mathbb{N}_{\geq 6}$ and $C_{n} \oplus C_{n}$ has Property $\mathbf{B}$, then $C_{2 n} \oplus C_{2 n}$ has Property $\mathbf{B}$ (also, simultaneously to this work, it was shown that $C_{3 n} \oplus C_{3 n}$ has Property B by Bhowmik, Halupczok and Schlage-Puchta, who did not publish their manuscript). Based on the above, the numerical verification of Property $\mathbf{B}$ for small $n \leq 10$ (for $n \leq 6$, see [9, Proposition 4.2]; the cases $n \in\{8,9,10\}$, and more, are settled in [2]), and a recent result of Schmid [21] on the structure of minimal zero-sum sequences in general groups of rank two, the above theorem implies that if $G=C_{n_{1}} \oplus C_{n_{2}}$ with $1<n_{1} \mid n_{2}$ is a group of rank two, and for every prime divisor $p$ of $n_{1}$ the group $C_{p} \oplus C_{p}$ has Property $\mathbf{B}$, then the minimal zero-sum sequences of maximal length over $G$ are explicitly characterized. More precisely, we have the following corollary.

Corollary. Let $G=C_{n_{1}} \oplus C_{n_{2}}$ with $1<n_{1} \mid n_{2}$ and suppose that, for every prime divisor $p$ of $n_{1}$, the group $C_{p} \oplus C_{p}$ has Property $\mathbf{B}$. Then $C_{n_{1}} \oplus C_{n_{1}}$ has Property $\mathbf{B}$, and a sequence $S$ over $G$ of length $\mathrm{D}(G)=$ $n_{1}+n_{2}-1$ is a minimal zero-sum sequence if and only if it has one of the following two forms:

$$
S=e_{1}^{\operatorname{ord}\left(e_{1}\right)-1} \prod_{\nu=1}^{\operatorname{ord}\left(e_{2}\right)}\left(-x_{\nu} e_{1}+e_{2}\right),
$$

where $\left\{e_{1}, e_{2}\right\}$ is a basis of $G, x_{1}, \ldots, x_{\operatorname{ord}\left(e_{2}\right)} \in\left[0, \operatorname{ord}\left(e_{1}\right)-1\right]$, and $x_{1}+$ $\cdots+x_{\text {ord }\left(e_{2}\right)} \equiv-1 \bmod \operatorname{ord}\left(e_{1}\right)$, or

$$
S=g_{1}^{s n_{1}-1} \prod_{\nu=1}^{n_{2}+(1-s) n_{1}}\left(-x_{\nu} g_{1}+g_{2}\right),
$$

where $\left\{g_{1}, g_{2}\right\}$ is a generating set of $G$ with $\operatorname{ord}\left(g_{2}\right)=n_{2}, s \in\left[1, n_{2} / n_{1}\right]$, $x_{1}, \ldots, x_{n_{2}+(1-s) n_{1}} \in\left[0, n_{1}-1\right], x_{1}+\cdots+x_{n_{2}+(1-s) n_{1}}=n_{1}-1$, and $(s=1$ or $\left.n_{1} g_{1}=n_{1} g_{2}\right)$. 
Thus the complete characterization of all minimal zero-sum sequences of length $\mathrm{D}(G)$ in groups of rank two is reduced to the verification of Property $\mathbf{B}$ in groups of the form $C_{p} \oplus C_{p}$ with $p$ prime. Property $\mathbf{B}$ is verified for small primes, and its validity, in general, is supported by other partial results (see [13, Section 5.2]). Much recent progress has been achieved by Bhowmik, Halupczok and Schlage-Puchta ([1, 2]).

In Section 2, we fix our notation and gather the necessary tools (apart from former work on Property $\mathbf{B}$ and classical addition theorems, we use a confirmed conjecture of Y. ould Hamidoune; see Theorem 2.7). Section 3 contains some straightforward lemmas. The proof of the Theorem consists of two major parts. The first is given in Section 4 . The second, more involved portion is given in Section 5. The Corollary follows from the results mentioned above, and its proof needs only a few lines and is given in Section 6 .

2. Preliminaries. Our notation and terminology are consistent with [11] and [14. We briefly gather some key notions and fix the notation concerning sequences over abelian groups. Let $\mathbb{N}$ denote the set of positive integers and let $\mathbb{N}_{0}=\mathbb{N} \cup\{0\}$. For real numbers $a, b \in \mathbb{R}$, we set $[a, b]=\{x \in \mathbb{Z} \mid a \leq x \leq b\}$. Throughout, all abelian groups will be written additively. For $n \in \mathbb{N}$, let $C_{n}$ denote a cyclic group with $n$ elements. Let $G$ be an abelian group.

Let $A, B \subset G$ be nonempty subsets. Then $A+B=\{a+b \mid a \in A, b \in B\}$ denotes their sumset and $A-B=\{a-b \mid a \in A, b \in B\}$ their difference set. The stabilizer of $A$ is defined as $\operatorname{Stab}(A)=\{g \in G \mid g+A=A\}$, and $A$ is called periodic if $\operatorname{Stab}(A) \neq\{0\}$.

An $s$-tuple $\left(e_{1}, \ldots, e_{s}\right)$ of elements of $G$ is said to be independent if $e_{i} \neq 0$ for all $i \in[1, s]$ and, for every $s$-tuple $\left(m_{1}, \ldots, m_{s}\right) \in \mathbb{Z}^{s}$,

$$
m_{1} e_{1}+\cdots+m_{s} e_{s}=0 \text { implies } m_{1} e_{1}=\cdots=m_{s} e_{s}=0 .
$$

An $s$-tuple $\left(e_{1}, \ldots, e_{s}\right)$ of elements of $G$ is called a basis if it is independent and $G=\left\langle e_{1}\right\rangle \oplus \cdots \oplus\left\langle e_{s}\right\rangle$.

Let $G=C_{n} \oplus C_{n}$ with $n \geq 2$, and let $\left(e_{1}, e_{2}\right)$ be a basis of $G$. An endomorphism $\varphi: G \rightarrow G$ with

$$
\left(\varphi\left(e_{1}\right), \varphi\left(e_{2}\right)\right)=\left(e_{1}, e_{2}\right) \cdot\left(\begin{array}{ll}
a & b \\
c & d
\end{array}\right), \quad \text { where } a, b, c, d \in \mathbb{Z},
$$

is an automorphism if and only if $\left(\varphi\left(e_{1}\right), \varphi\left(e_{2}\right)\right)$ is a basis, which is equivalent to $\operatorname{gcd}(a d-b c, n)=1$. If $f_{1} \in G$ with $\operatorname{ord}\left(f_{1}\right)=n$, then clearly there is an $f_{2} \in G$ such that $\left(f_{1}, f_{2}\right)$ is a basis of $G$. 
Let $\mathcal{F}(G)$ be the free abelian monoid with basis $G$. The elements of $\mathcal{F}(G)$ are called sequences over $G$. We write sequences $S \in \mathcal{F}(G)$ in the form

$$
S=\prod_{g \in G} g^{\mathrm{v}_{g}(S)} \quad \text { with } \mathrm{v}_{g}(S) \in \mathbb{N}_{0} \text {, and } \mathrm{v}_{g}(S)=0 \text { for almost all } g \in G .
$$

We call $\vee_{g}(S)$ the multiplicity of $g$ in $S$, and we say that $S$ contains $g$ if $\mathrm{v}_{g}(S)>0$. A sequence $S_{1}$ is called a subsequence of $S$ if $S_{1} \mid S$ in $\mathcal{F}(G)$ (equivalently, $\mathrm{v}_{g}\left(S_{1}\right) \leq \mathrm{v}_{g}(S)$ for all $g \in G$ ). Note that for two sequences $S, T \in \mathcal{F}(G), \operatorname{gcd}(S, T)$ is the longest subsequence dividing both $S$ and $T$. If a sequence $S \in \mathcal{F}(G)$ is written in the form $S=g_{1} \cdot \ldots \cdot g_{l}$, we tacitly assume that $l \in \mathbb{N}_{0}$ and $g_{1}, \ldots, g_{l} \in G$.

For a sequence

$$
S=g_{1} \cdot \ldots \cdot g_{l}=\prod_{g \in G} g^{v_{g}(S)} \in \mathcal{F}(G),
$$

we call

- $|S|=l=\sum_{g \in G} \mathrm{v}_{g}(S) \in \mathbb{N}_{0} \quad$ the length of $S$,

- $\mathrm{h}(S)=\max \left\{\mathrm{v}_{g}(S) \mid g \in G\right\} \in[0,|S|]$ the maximum of the multiplicities of $S$,

- $\operatorname{supp}(S)=\left\{g \in G \mid \mathrm{v}_{g}(S)>0\right\} \subset G$ the support of $S$,

- $\sigma(S)=\sum_{i=1}^{l} g_{i}=\sum_{g \in G} \mathrm{v}_{g}(S) g \in G$ the sum of $S$,

- $\Sigma_{k}(S)=\left\{\sum_{i \in I} g_{i} \mid I \subset[1, l]\right.$ with $\left.|I|=k\right\}$ the set of $k$-term subsums of $S$, for all $k \in \mathbb{N}$,

- $\Sigma_{\leq k}(S)=\bigcup_{j \in[1, k]} \Sigma_{j}(S), \quad \Sigma_{\geq k}(S)=\bigcup_{j \geq k} \Sigma_{j}(S)$,

- $\Sigma(S)=\Sigma_{\geq 1}(S)$ the set of (all) subsums of $S$.

The sequence $S$ is called

- zero-sum free if $0 \notin \Sigma(S)$,

- a zero-sum sequence if $\sigma(S)=0$,

- a minimal zero-sum sequence if $1 \neq S, \sigma(S)=0$, and every $S^{\prime} \mid S$ with $1 \leq\left|S^{\prime}\right|<|S|$ is zero-sum free.

We denote by $\mathcal{A}(G) \subset \mathcal{F}(G)$ the set of all minimal zero-sum sequences over $G$. Every map of abelian groups $\varphi: G \rightarrow H$ extends to a homomorphism $\varphi: \mathcal{F}(G) \rightarrow \mathcal{F}(H)$ where $\varphi(S)=\varphi\left(g_{1}\right) \cdot \ldots \cdot \varphi\left(g_{l}\right)$. We say that $\varphi$ is constant on $S$ if $\varphi\left(g_{1}\right)=\cdots=\varphi\left(g_{l}\right)$. If $\varphi$ is a homomorphism, then $\varphi(S)$ is a zero-sum sequence if and only if $\sigma(S) \in \operatorname{Ker}(\varphi)$. 
Definition 2.1. Let $G$ be a finite abelian group with exponent $n$.

1. Let $\mathrm{D}(G)$ denote the smallest integer $\ell \in \mathbb{N}$ such that every sequence $S \in \mathcal{F}(G)$ of length $|S| \geq \ell$ has a nontrivial zero-sum subsequence. Equivalently, we have $\mathrm{D}(G)=\max (\{|S| \mid S \in \mathcal{A}(G)\})$, and $\mathrm{D}(G)$ is called the Davenport constant of $G$.

2. Let $\eta(G)$ denote the smallest integer $\ell \in \mathbb{N}$ such that every sequence $S \in \mathcal{F}(G)$ of length $|S| \geq \ell$ has a zero-sum subsequence $T$ of length $|T| \in[1, n]$.

3. We say that $G$ has Property $\mathbf{C}$ if every sequence $S$ over $G$ of length $|S|=\eta(G)-1$, with no zero-sum subsequence of length in $[1, n]$, has the form $S=T^{n-1}$ for some sequence $T$ over $G$.

Lemma 2.2. Let $G=C_{n_{1}} \oplus C_{n_{2}}$ with $1 \leq n_{1} \mid n_{2}$.

1. We have $\mathrm{D}(G)=n_{1}+n_{2}-1$ and $\eta(G)=2 n_{1}+n_{2}-2$.

2. If $n_{1}=n_{2}$ and $G$ has Property $\mathbf{B}$, then $G$ has Property $\mathbf{C}$.

Proof. 1. See [14, Theorem 5.8.3].

2. See [9, Theorem 6.2] and [10, Theorem 6.7.2(b)].

Results on $\eta(G)$ for groups of higher rank may be found in [6, 5, 12, 4, 22.

Lemma 2.3. Let $G=C_{n} \oplus C_{n}$ with $n \geq 2$.

1. The following statements are equivalent:

(a) If $S \in \mathcal{F}(G),|S|=3 n-3$ and $S$ has no zero-sum subsequence $T$ of length $|T| \geq n$, then there exists some $a \in G$ such that $0^{n-1} a^{n-2} \mid S$.

(b) If $S \in \mathcal{F}(G)$ is zero-sum free and $|S|=2 n-2$, then $a^{n-2} \mid S$ for some $a \in G$.

(c) G has Property B. Namely, if $S \in \mathcal{A}(G)$ and $|S|=2 n-1$, then $a^{n-1} \mid S$ for some $a \in G$.

(d) If $S \in \mathcal{A}(G)$ and $|S|=2 n-1$, then there exists a basis $\left(e_{1}, e_{2}\right)$ of $G$ and integers $x_{1}, \ldots, x_{n} \in[0, n-1]$, with $x_{1}+\cdots+x_{n} \equiv 1 \bmod n$, such that

$$
S=e_{1}^{n-1} \prod_{\nu=1}^{n}\left(x_{\nu} e_{1}+e_{2}\right) .
$$

2. Let $S \in \mathcal{A}(G)$ be of length $|S|=2 n-1$ and $e_{1} \in G$ with $\mathrm{v}_{e_{1}}(S)=n-1$. If $\left(e_{1}, e_{2}^{\prime}\right)$ is a basis of $G$, then there exist some $b \in[0, n-1]$ and $a_{1}^{\prime}, \ldots, a_{n}^{\prime} \in[0, n-1]$, with $\operatorname{gcd}(b, n)=1$ and $\sum_{\nu=1}^{n} a_{\nu}^{\prime} \equiv 1 \bmod n$, such that

$$
S=e_{1}^{n-1} \prod_{\nu=1}^{n}\left(a_{\nu}^{\prime} e_{1}+b e_{2}^{\prime}\right) .
$$


3. If $S \in \mathcal{A}(G)$ has length $|S|=2 n-1$, then $\operatorname{ord}(g)=n$ for all $g \in$ $\operatorname{supp}(S)$.

Proof. 1. See [14, Theorem 5.8.7].

2. This follows easily from item 1 ; for details see [9, Proposition 4.1].

3. See [14, Theorem 5.8.4].

The characterization in Lemma 2.3.1 gives rise to the following definition.

Definition 2.4. Let $G=C_{n} \oplus C_{n}$ with $n \geq 2$.

1. Let $\Upsilon(G)$ be the set of all $S \in \mathcal{A}(G)$ for which there exists a basis $\left(e_{1}, e_{2}\right)$ of $G$ and integers $x_{1}, \ldots, x_{n} \in[0, n-1]$, with $x_{1}+\cdots+x_{n} \equiv$ $1 \bmod n$, such that $S=e_{1}^{n-1} \prod_{\nu=1}^{n}\left(x_{\nu} e_{1}+e_{2}\right)$.

2. Let $\Upsilon_{u}(G)$ be the set of those $S \in \Upsilon(G)$ with a unique term of multiplicity $n-1$, and let $\Upsilon_{n u}(G)=\Upsilon(G) \backslash \Upsilon_{u}(G)$ be those $S \in \Upsilon(G)$ with a nonunique term of multiplicity $n-1$.

Thus, by Lemma 2.3 1, a group $G=C_{n} \oplus C_{n}$ with $n \geq 2$ has Property B if and only if $\{S \in \mathcal{A}(G)|| S \mid=2 n-1\}=\Upsilon(G)$.

Lemma 2.5. Let $G=C_{m n} \oplus C_{m n}$ with $m, n \geq 2$, let $S \in \mathcal{A}(G)$ be of length $|S|=2 m n-1$, and let $\varphi: G \rightarrow G$ denote the multiplication by $m$ homomorphism.

1. $\varphi(S)$ is not a product of $2 m$ zero-sum subsequences. Every zero-sum subsequence $T$ of $\varphi(S)$ of length $|T| \in[1, n]$ has length $n$, and $0 \notin$ $\operatorname{supp}(\varphi(S))$.

2. $S$ may be written in the form $S=W_{0} \cdot \ldots \cdot W_{2 m-2}$, where $W_{0}, \ldots$, $W_{2 m-2} \in \mathcal{F}(G)$ with $\left|W_{0}\right|=2 n-1,\left|W_{1}\right|=\cdots=\left|W_{2 m-2}\right|=n$ and $\sigma\left(W_{0}\right), \ldots, \sigma\left(W_{2 m-2}\right) \in \operatorname{Ker}(\varphi)$.

Proof. See [9, Lemma 3.14].

The following is the Erdős-Ginzburg-Ziv Theorem and the corresponding characterization of extremal sequences. There are much stronger inverse results (see [13, Section 5]), but the one mentioned below will be sufficient for our purposes.

Theorem 2.6. Let $G$ be a cyclic group of order $n \geq 2$ and $S \in \mathcal{F}(G)$.

1. If $|S| \geq 2 n-1$, then $0 \in \Sigma_{n}(S)$.

2. If $|S|=2 n-2$ and $0 \notin \Sigma_{n}(S)$, then $S=g^{n-1} h^{n-1}$ for some $g, h \in G$ with $\operatorname{ord}(g-h)=n$.

Proof. 1. See [14, Corollary 5.7.5] or [18, Theorem 2.5].

2. See [3, Lemma 4] for one of the original proofs, and [13, Proposition $5.1 .12]$.

The following result was a conjecture of Y. ould Hamidoune [17] confirmed in [16, Theorem 1]. 
TheOREM 2.7. Let $G$ be a finite abelian group, $S \in \mathcal{F}(G)$ of length $|S| \geq|G|+1$, and $k \in \mathbb{N}$ with $k \leq|\operatorname{supp}(S)|$. If $\mathrm{h}(S) \leq|G|-k+2$ and $0 \notin \Sigma_{|G|}(S)$, then $\left|\Sigma_{|G|}(S)\right| \geq|S|-|G|+k-1$.

3. Preparatory results. We first prove several lemmas determining in what ways a sequence $S \in \Upsilon\left(C_{m} \oplus C_{m}\right)$, where $m \geq 4$, can be slightly perturbed and still remain in $\Upsilon\left(C_{m} \oplus C_{m}\right)$. These will later be heavily used in Section 5, always in the setting where $K=\operatorname{Ker}(\varphi)$ and $\varphi: G \rightarrow G$ is multiplication by $m$.

Lemma 3.1. Let $K=C_{m} \oplus C_{m}$ with $m \geq 4$, let $g \in K$, and let $S=$ $f_{1}^{m-1} \prod_{\nu=1}^{m}\left(x_{\nu} f_{1}+f_{2}\right) \in \Upsilon_{u}(K)$ with $x_{1}, \ldots, x_{m} \in \mathbb{Z}$.

1. If $S^{\prime}=f_{1}^{-2} S\left(f_{1}+g\right)\left(f_{1}-g\right) \in \Upsilon(K)$, then $g=0$ and hence $S=S^{\prime}$.

2. If $S^{\prime}=f_{1}^{-1}\left(x_{j} f_{1}+f_{2}\right)^{-1} S\left(f_{1}+g\right)\left(x_{j} f_{1}+f_{2}-g\right) \in \Upsilon(K)$, then $g \in\left\{0,\left(x_{j}-1\right) f_{1}+f_{2}\right\}$ and hence $S=S^{\prime}$.

3. If $S^{\prime}=\left(x_{j} f_{1}+f_{2}\right)^{-1}\left(x_{k} f_{1}+f_{2}\right)^{-1} S\left(x_{j} f_{1}+f_{2}+g\right)\left(x_{k} f_{1}+f_{2}-g\right) \in \Upsilon(K)$ with $j, k \in[1, m]$ distinct, then $g \in\left\langle f_{1}\right\rangle$.

Proof. 1. Assume to the contrary that $g \neq 0$ and thus $S \neq S^{\prime}$. Then $\mathrm{v}_{f_{1}}\left(S^{\prime}\right)<m-1$ and, since $m \geq 4, S^{\prime} \in \Upsilon(k)$ and $S \in \Upsilon_{u}(K)$, it follows that there is some $j \in[1, m]$ such that $\left(x_{j} f_{1}+f_{2}\right)^{m-1}\left|S^{\prime},\left(x_{j} f_{1}+f_{2}\right)^{m-3}\right| S$, and w.l.o.g. $x_{j} f_{1}+f_{2}=f_{1}+g$. If we set $f_{2}^{\prime}=x_{j} f_{1}+f_{2}$, then $S=f_{1}^{m-1} \prod_{\nu=1}^{m}\left(\left(x_{\nu}-\right.\right.$ $\left.x_{j}\right) f_{1}+f_{2}^{\prime}$ ), and thus we may assume that $f_{2}=f_{2}^{\prime}$. Then $f_{2}=f_{1}+g$ and $f_{1}-g=f_{2}-2 g=2 f_{1}-f_{2}$. Since $m \geq 4$, it follows that $f_{1} \mid S^{\prime}$. Since $S^{\prime} \in \Upsilon(K), f_{2}^{m-1} \mid S^{\prime}$ and $f_{1}, 2 f_{1}-f_{2} \in \operatorname{supp}\left(S^{\prime}\right) \backslash\left\{f_{2}\right\}$, it follows that $\left(2 f_{1}-f_{2}\right)-f_{1}=f_{1}-f_{2} \in\left\langle f_{2}\right\rangle$, contradicting that $\left(f_{1}, f_{2}\right)$ is a basis.

2. After renumbering, we may suppose that $j=m$. If $f_{1}^{m-1} \mid S^{\prime}$, then $f_{1}+g=f_{1}$ or $x_{m} f_{1}+f_{2}-g=f_{1}$, and $S^{\prime}=S$. Otherwise, $f_{1}^{m-1} \nmid S^{\prime}$ and we shall derive a contradiction. Observe that we cannot have $f_{1}+g=$ $x_{m} f_{1}+f_{2}-g=x_{i} f_{1}+f_{2}$, else $g, f_{2} \in\left\langle f_{1}\right\rangle$. Thus, since $S^{\prime} \in \Upsilon(K), S \in \Upsilon_{u}(K)$ and $m \geq 4$, it follows that (after renumbering again if necessary) either $S^{\prime}=f_{1}^{m-2}\left(x f_{1}+f_{2}\right)^{m-1}\left(x_{m} f_{1}+f_{2}-g\right)\left(x_{m-1} f_{1}+f_{2}\right) \quad$ with $f_{1}+g=x f_{1}+f_{2}$, or $S^{\prime}=f_{1}^{m-2}\left(x f_{1}+f_{2}\right)^{m-1}\left(f_{1}+g\right)\left(x_{m-1} f_{1}+f_{2}\right) \quad$ with $x_{m} f_{1}+f_{2}-g=x f_{1}+f_{2}$. In the first case, we have $\left(x_{m} f_{1}+f_{2}-g\right)=\left(x_{m}-x+1\right) f_{1}$ and hence $f_{1}^{m-2}\left(\left(x_{m}-x+1\right) f_{1}\right) \mid S^{\prime}$. However, since $\left(x_{m}-x+1\right) f_{1}=\left(x_{m} f_{1}+f_{2}-g\right) \neq f_{1}$ (else $g=\left(x_{n}-1\right) f_{1}+f_{2}$, as desired), it follows that $f_{1}^{m-2}\left(\left(x_{m}-x+1\right) f_{1}\right)$ is not zero-sum free (as $f_{1}$ is the unique element from $\left\langle f_{1}\right\rangle$ that can be appended to $f_{1}^{n-1}$ without yielding a zero-sum), a contradiction. In the second case, one can derive a contradiction similarly. 
3. Since $m \geq 3, f_{1}^{m-1} \mid S^{\prime}$ and $S^{\prime} \in \Upsilon(K)$, it follows that $\left(x_{j} f_{1}+f_{2}+g\right)$ $-\left(x_{l} f_{1}+f_{2}\right) \in\left\langle f_{1}\right\rangle$, where $l \neq j, k$, and hence $g \in\left\langle f_{1}\right\rangle$.

Lemma 3.2. Let $K=C_{m} \oplus C_{m}$ with $m \geq 4, g \in K$ and $S=$ $f_{1}^{m-1} f_{2}^{m-1}\left(f_{1}+f_{2}\right) \in \Upsilon_{n u}(K)$.

1. If $S^{\prime}=f_{1}^{-2} S\left(f_{1}+g\right)\left(f_{1}-g\right) \in \Upsilon(K)$, then $g \in\left\langle f_{2}\right\rangle$.

2. If $S^{\prime}=f_{2}^{-2} S\left(f_{2}+g\right)\left(f_{2}-g\right) \in \Upsilon(K)$, then $g \in\left\langle f_{1}\right\rangle$.

3. If $S^{\prime}=f_{1}^{-1} f_{2}^{-1} S\left(f_{1}+g\right)\left(f_{2}-g\right) \in \Upsilon(K)$, then $S=S^{\prime}$ and $g \in$ $\left\{0,-f_{1}+f_{2}\right\}$.

4. If $S^{\prime}=f_{1}^{-1}\left(f_{1}+f_{2}\right)^{-1} S\left(f_{1}+g\right)\left(f_{1}+f_{2}-g\right) \in \Upsilon(K)$, then $g \in\left\langle f_{2}\right\rangle$.

5. If $S^{\prime}=f_{2}^{-1}\left(f_{1}+f_{2}\right)^{-1} S\left(f_{2}+g\right)\left(f_{1}+f_{2}-g\right) \in \Upsilon(K)$, then $g \in\left\langle f_{1}\right\rangle$.

Proof. 1. Since $f_{2}^{m-1} \mid S^{\prime}$ and $S^{\prime} \in \Upsilon(K)$, it follows that $f_{1}+g-\left(f_{1}+f_{2}\right)$ $\in\left\langle f_{2}\right\rangle$, whence $g \in\left\langle f_{2}\right\rangle$.

2. Analogous to the proof of item 1.

3. If $f_{1}^{m-1} \mid S^{\prime}$ or $f_{2}^{m-1} \mid S^{\prime}$, the result follows. Otherwise, $m \geq 4$ and $\mathrm{h}\left(S^{\prime}\right)=m-1$ imply that $m=4$ and $f_{1}+g=f_{2}-g=f_{1}+f_{2}$, a contradiction.

4. Since $m \geq 3$, it follows that $f_{1} \mid S^{\prime}$. Now we have $f_{2}^{m-1} \mid S^{\prime}$ and $S^{\prime} \in$ $\Upsilon(K)$ so that $\left(f_{1}+f_{2}-g\right)-f_{1} \in\left\langle f_{2}\right\rangle$, implying $g \in\left\langle f_{2}\right\rangle$, as desired.

5. Analogous to the proof of item 4 .

Lemma 3.3. Let $K=C_{m} \oplus C_{m}$ with $m \geq 4, g \in K$ and $S=$ $f_{1}^{m-1} f_{2}^{m-1}\left(f_{1}+f_{2}\right) \in \Upsilon_{n u}(K)$.

1. If $S^{\prime}=f_{1}^{-2} S\left(f_{1}+g\right)\left(f_{1}-g\right) \in \Upsilon_{n u}(K)$, then $g=0$, and hence $S=S^{\prime}$.

2. If $S^{\prime}=f_{2}^{-2} S\left(f_{2}+g\right)\left(f_{2}-g\right) \in \Upsilon_{n u}(K)$, then $g=0$, and hence $S=S^{\prime}$.

3. If $S^{\prime}=f_{1}^{-1} f_{2}^{-1} S\left(f_{1}+g\right)\left(f_{2}-g\right) \in \Upsilon_{n u}(K)$, then $g \in\left\{0,-f_{1}+f_{2}\right\}$, and hence $S=S^{\prime}$.

4. If $S^{\prime}=f_{1}^{-1}\left(f_{1}+f_{2}\right)^{-1} S\left(f_{1}+g\right)\left(f_{1}+f_{2}-g\right) \in \Upsilon_{n u}(K)$, then $g \in\left\{0, f_{2}\right\}$, and hence $S=S^{\prime}$.

5. If $S^{\prime}=f_{2}^{-1}\left(f_{1}+f_{2}\right)^{-1} S\left(f_{2}+g\right)\left(f_{1}+f_{2}-g\right) \in \Upsilon_{n u}(K)$, then $g \in\left\{0, f_{1}\right\}$, and hence $S=S^{\prime}$.

Proof. 1. Assume to the contrary that $g \neq 0$ and $S \neq S^{\prime}$. Since $S^{\prime} \in$ $\Upsilon_{n u}(K)$ and $m \geq 4$, we get $f_{1}+g=f_{1}-g=f_{1}+f_{2}$ and hence $-2 f_{2}=2 g=0$, a contradiction.

2.-5. Similar.

Next we prove two simple structural lemmas which will be our allpurpose tools for turning locally obtained information into global structural conditions on $S$. They are also the reason for the hypothesis of $m$ and $n$ odd in the Theorem.

Lemma 3.4. Let $G$ be an abelian group, $a \in G$ with $\operatorname{ord}(a)>2$, and $S, T \in \mathcal{F}(G) \backslash\{1\}$ with $|\operatorname{supp}(S)| \geq|\operatorname{supp}(T)|$. 
1. If $\operatorname{supp}(S)-\operatorname{supp}(T)=\{0\}$, then $S=g^{|S|}$ and $T=g^{|T|}$, for some $g \in G$.

2. If $\operatorname{supp}(S)-\operatorname{supp}(T) \subset\{0, a\}$, then $S=g^{s}(g+a)^{|S|-s}$ and $T=g^{|T|}$, for some $g \in G$ and $s \in[0,|S|]$.

3. If $|S|,|T| \geq 2$ and $\bigcup_{i=1}^{2}\left(\Sigma_{i}(S)-\Sigma_{i}(T)\right) \subset\{0, a\}$, then either $S=$ $g^{|S|-1}(g+a)$ and $T=g^{|T|}$, or else $S=g^{|S|}$ and $T=g^{|T|}$, for some $g \in G$.

Proof. Note that $\Sigma_{1}(S)=\operatorname{supp}(S)$ and that all hypotheses imply $\operatorname{supp}(S)-\operatorname{supp}(T) \subset\{0, a\}$. Since $\operatorname{ord}(a)>2$, it follows that $\{0, a\}$ contains no periodic subset, and thus Kneser's Theorem (see e.g., [14, Theorem 5.2.6]) implies that

$$
2 \geq|\operatorname{supp}(S)-\operatorname{supp}(T)| \geq|\operatorname{supp}(S)|+|\operatorname{supp}(T)|-1 .
$$

Therefore we get $|\operatorname{supp}(S)| \leq 2$ and $|\operatorname{supp}(T)|=1$. Items 1 and 2 now easily follow. For the proof of part 3 , we apply assertion 2 , and thus we may assume that $\operatorname{supp}(S) \subset\{g, g+a\}$ and $T=g^{|T|}$. Now if item 3 is false, then $(g+a)^{2} \mid S$, whence

$$
2 a=((g+a)+(g+a))-(g+g) \in \bigcup_{i=1}^{2}\left(\Sigma_{i}(S)-\Sigma_{i}(T)\right) \subset\{0, a\},
$$

contradicting $\operatorname{ord}(a)>2$.

Lemma 3.5. Let $G$ be an abelian group and let $S \in \mathcal{F}(G)$.

1. If $k \in[1,|S|-1]$ and $\left|\Sigma_{k}(S)\right| \leq 2$, then $|\operatorname{supp}(S)| \leq 2$.

2. If $k \in[2,|S|-2]$ and $\left|\Sigma_{k}(S)\right| \leq 2$, and $\Sigma_{k}(S)$ is not a coset of a cardinality two subgroup, then either $S=g^{|S|}$ or $S=g^{|S|-1} h$, for some $g, h \in G$.

3. If $k \in[1,|S|-1]$ and $\left|\Sigma_{k}(S)\right| \leq 1$, then $S=g^{|S|}$ for some $g \in G$.

Proof. 1. Assume to the contrary that $|\operatorname{supp}(S)| \geq 3$ and pick three distinct elements $x, y, z \in \operatorname{supp}(S)$. If $k=|S|-1$, then $\Sigma_{|S|-1}(S)=\sigma(S)-$ $\Sigma_{1}(S)$ and hence $\left|\Sigma_{|S|-1}(S)\right|=|\operatorname{supp}(S)| \geq 3$, a contradiction. Therefore $k \leq|S|-2$. Let $T$ be a subsequence (possibly trivial) of $(x y z)^{-1} S$ of length $|T|=k-1 \leq|S|-3$. Then $\{x, y, z\}+\sigma(T)$ is a cardinality three subset of $\Sigma_{k}(S)$, a contradiction.

2. By item 1, we have $S=g^{s_{1}} h^{s_{2}}$ with $s_{1}, s_{2} \in \mathbb{N}_{0}, s_{1} \geq s_{2}$ and $g, h \in G$ distinct. Assume to the contrary that $s_{2} \geq 2$. Since $\Sigma_{|S|-k}(S)=\sigma(S)-$ $\Sigma_{k}(S)$, it suffices to consider the case $k \leq \frac{1}{2}|S|$, and thus $s_{1} \geq \frac{1}{2}|S| \geq k \geq 2$. Hence the elements $k g,(k-1) g+h$ and $(k-2) g+2 h$ are all contained in $\Sigma_{k}(S)$. Thus, since $\left|\Sigma_{k}(S)\right| \leq 2$ and $g \neq h$, it follows that $\operatorname{ord}(h-g)=2$ and $\Sigma_{k}(S)=k g+\{0, h-g\}$, contradicting the assumption that $\Sigma_{k}(S)$ is not a coset of a cardinality two subgroup. 
3. If the conclusion is false, there are distinct $x, y \in G$ with $x y \mid S$, and then $\{x, y\}+\sigma\left(S^{\prime}\right)$ is a cardinality two subset of $\Sigma_{k}(S)$ for any $S^{\prime} \mid(x y)^{-1} S$ with $0 \leq\left|S^{\prime}\right|=k-1 \leq|S|-2$.

\section{On the structure of $\varphi(S)$}

Definition 4.1. Let $G=C_{m n} \oplus C_{m n}$ with $m, n \geq 2$, let $S \in \mathcal{A}(G)$ with $|S|=2 m n-1$, and let $\varphi: G \rightarrow G$ be multiplication by $m$. Let

$$
\begin{array}{r}
\Omega^{\prime}(S)=\Omega^{\prime}=\left\{\left(W_{0}, \ldots, W_{2 m-2}\right) \in \mathcal{F}(G)^{2 m-1} \mid S=W_{0} \cdot \ldots \cdot W_{2 m-2},\right. \\
\left.\sigma\left(W_{i}\right) \in \operatorname{Ker}(\varphi) \text { and }\left|W_{i}\right|>0 \text { for all } i \in[0,2 m-2]\right\}
\end{array}
$$

and

$$
\Omega(S)=\Omega=\left\{\left(W_{0}, \ldots, W_{2 m-2}\right) \in \Omega^{\prime}|| W_{1}|=\cdots=| W_{2 m-2} \mid=n\right\} .
$$

The elements $\left(W_{0}, \ldots, W_{2 m-2}\right) \in \Omega^{\prime}(S)$ will be called product decompositions of $S$. If $W \in \Omega^{\prime}$, we implicitly assume that $W=\left(W_{0}, \ldots, W_{2 m-2}\right)$.

By Lemma 2.5, $\Omega \neq \emptyset$, and if $W \in \Omega$, then $\varphi\left(W_{0}\right), \ldots, \varphi\left(W_{2 m-2}\right)$ are minimal zero-sum sequences over $\varphi(G) \cong C_{n} \oplus C_{n}$. Proposition 4.2 below shows that $\varphi(S)$ is highly structured. In Claims A, B and C of Section 5 we will show (with much effort) that this structure lifts to the original sequence $S$. As this lift will only be "near perfect" (there will be one exceptional term $x \mid S$ for which the structure is not shown to lift), we will then, in Claim D of Section 5, need Theorem 2.7 to finish the proof of the Theorem.

Proposition 4.2. Let $G=C_{m n} \oplus C_{m n}$ with $m, n \geq 2$, and suppose that $C_{n} \oplus C_{n}$ has Property B. Let $S \in \mathcal{A}(G)$ with $|S|=2 m n-1$, and let $\varphi: G \rightarrow G$ be multiplication by $m$. Then there exist a product decomposition $\left(W_{0}, \ldots, W_{2 m-2}\right)$ of $S$ and a basis $\left(e_{1}, e_{2}\right)$ of $\varphi(G)$ such that

$$
\varphi\left(W_{0}\right)=e_{1}^{n-1} \prod_{\nu=1}^{n}\left(x_{\nu} e_{1}+e_{2}\right) \quad \text { and } \quad \varphi\left(W_{i}\right) \in\left\{e_{1}^{n}, \prod_{\nu=1}^{n}\left(c_{i, \nu} e_{1}+e_{2}\right)\right\},
$$

where $x_{1}, \ldots, x_{n} \in[0, n-1], x_{1}+\cdots+x_{n} \equiv 1 \bmod n$, all $c_{i, \nu} \in[0, n-1]$, and $c_{i, 1}+c_{i, 2}+\cdots+c_{i, n} \equiv 0 \bmod n$ for all $i \in[1, n]$. In particular,

$$
\varphi(S)=e_{1}^{\ell n-1} \prod_{\nu=1}^{2 m n-\ell n}\left(x_{\nu} e_{1}+e_{2}\right),
$$

where $\ell \in[1,2 m-1]$ and $x_{\nu} \in[0, n-1]$ for all $\nu \in[1,2 m n-\ell n]$.

Proof. If $n=2$, then it is easy to see (in view of Lemma 2.5) that (1) holds. From now on we assume that $n \geq 3$. We distinguish two cases.

CASE 1: For every product decomposition $W \in \Omega$, there exist distinct elements $g_{1}, g_{2} \in \varphi(G)$ such that $\mathrm{v}_{g_{1}}\left(\varphi\left(W_{0}\right)\right)=\mathrm{v}_{g_{2}}\left(\varphi\left(W_{0}\right)\right)=n-1$. Let us 
fix a product decomposition $W \in \Omega$. By Lemma 2.3 , there is a basis $\left(e_{1}, e_{2}^{\prime}\right)$ of $\varphi(G)$ such that

$$
\varphi\left(W_{0}\right)=e_{1}^{n-1} \prod_{\nu=1}^{n}\left(x_{\nu} e_{1}+e_{2}^{\prime}\right)
$$

where $x_{1}, \ldots, x_{n} \in[0, n-1]$ and $x_{1}+\cdots+x_{n} \equiv 1 \bmod n$. Thus, by assumption of Case 1, it follows that

$$
\varphi\left(W_{0}\right)=e_{1}^{n-1}\left(x e_{1}+e_{2}^{\prime}\right)^{n-1}\left((1+x) e_{1}+e_{2}^{\prime}\right) \quad \text { with } x \in[0, n-1] .
$$

As a result,

$$
\left(e_{1}, e_{2}\right)=\left(e_{1}, x e_{1}+e_{2}^{\prime}\right)=\left(e_{1}, e_{2}^{\prime}\right) \cdot\left(\begin{array}{ll}
1 & x \\
0 & 1
\end{array}\right)
$$

is a basis of $\varphi(G)$ and

$$
\varphi\left(W_{0}\right)=e_{1}^{n-1} e_{2}^{n-1}\left(e_{1}+e_{2}\right) .
$$

We continue with the following assertion.

A. For every $i \in[1,2 m-2], \varphi\left(W_{i}\right)$ has one of the following forms:

$$
\begin{aligned}
e_{1}^{n}, e_{2}^{n},\left(e_{1}+e_{2}\right)^{n}, & \left(-e_{1}+e_{2}\right)^{n},\left(e_{1}-e_{2}\right)^{n}, \\
& e_{1}\left(e_{1}+e_{2}\right)^{n-2}\left(e_{1}+2 e_{2}\right), e_{2}\left(e_{1}+e_{2}\right)^{n-2}\left(2 e_{1}+e_{2}\right) .
\end{aligned}
$$

Suppose that $\mathbf{A}$ is proved. If the forms $\left(e_{1}-e_{2}\right)^{n}$ and $e_{1}\left(e_{1}+e_{2}\right)^{n-2}\left(e_{1}+2 e_{2}\right)$ do not occur, then $\varphi\left(W_{i}\right)$ has the required form with basis $\left(e_{1}, e_{2}\right)$. If the forms $\left(-e_{1}+e_{2}\right)^{n}$ and $e_{2}\left(e_{1}+e_{2}\right)^{n-2}\left(2 e_{1}+e_{2}\right)$ do not occur, then $\varphi\left(W_{i}\right)$ has the required form with basis $\left(e_{2}, e_{1}\right)$. Thus by symmetry, it remains to verify that there are no distinct $i, j \in[1,2 m-2]$ such that

(i) $\varphi\left(W_{i}\right)=e_{1}\left(e_{1}+e_{2}\right)^{n-2}\left(e_{1}+2 e_{2}\right)$ and $\varphi\left(W_{j}\right)=e_{2}\left(e_{1}+e_{2}\right)^{n-2}\left(2 e_{1}+e_{2}\right)$,

(ii) $\varphi\left(W_{i}\right)=e_{1}\left(e_{1}+e_{2}\right)^{n-2}\left(e_{1}+2 e_{2}\right)$ and $\varphi\left(W_{j}\right)=\left(-e_{1}+e_{2}\right)^{n}$, or

(iii) $\varphi\left(W_{i}\right)=\left(e_{1}-e_{2}\right)^{n}$ and $\varphi\left(W_{j}\right)=\left(-e_{1}+e_{2}\right)^{n}$.

Indeed, if (i) held, then $\left(2 e_{1}+e_{2}\right)\left(e_{1}+2 e_{2}\right)\left(e_{1}+e_{2}\right)^{n-3}$ would be a zero-sum subsequence of $\varphi\left(W_{i} W_{j}\right)$ of length $n-1$, contradicting Lemma 2.5. If (ii) held, then $\left(-e_{1}+e_{2}\right)\left(e_{1}+2 e_{2}\right) e_{2}^{n-3}$ would be a zero-sum subsequence of $\varphi\left(W_{0} W_{i} W_{j}\right)$ of length $n-1$, contradicting Lemma 2.5. Finally, if (iii) held, then $\left(e_{1}-e_{2}\right)\left(-e_{1}+e_{2}\right)$ would be a zero-sum subsequence of $\varphi\left(W_{i} W_{j}\right)$ of length 2, also contradicting Lemma 2.5. Thus it remains to establish $\mathbf{A}$ to complete the case. To that end, let $i \in[1,2 m-2]$ be arbitrary. Then $\mathrm{h}\left(\varphi\left(W_{0} W_{i}\right)\right) \geq n-1$, and we distinguish three subcases.

CASE 1.1: $\mathrm{h}\left(\varphi\left(W_{0} W_{i}\right)\right)>n$. Then it follows from (2) that $\mathrm{v}_{g}\left(\varphi\left(W_{0} W_{i}\right)\right)$ $>n$ for some $g \in\left\{e_{1}, e_{2}, e_{1}+e_{2}\right\}$. If $g=e_{1}+e_{2}$, then $\varphi\left(W_{i}\right)=\left(e_{1}+e_{2}\right)^{n}$. 
Now suppose that $g \in\left\{e_{1}, e_{2}\right\}$, say $g=e_{1}$. Then

$$
\varphi\left(W_{0} W_{i}\right)=e_{2}^{n-1}\left(e_{1}+e_{2}\right) e_{1}^{n+1} \prod_{\nu=1}^{n-2}\left(c_{\nu} e_{1}+d_{\nu} e_{2}\right),
$$

where $c_{\nu}, d_{\nu} \in[0, n-1]$ for all $\nu \in[1, n-1]$. By Lemma 2.5.

$$
W_{0}^{\prime}=e_{2}^{n-1}\left(e_{1}+e_{2}\right) e_{1} \prod_{\nu=1}^{n-2}\left(c_{\nu} e_{1}+d_{\nu} e_{2}\right)
$$

is a minimal zero-sum subsequence of $\varphi(S)$. Since $W_{0}^{\prime}$ contains two distinct elements with multiplicity $n-1$ (by assumption of Case 1), and since $e_{1} \mid W_{0}^{\prime}$, it follows that either

$$
W_{0}^{\prime}=e_{1}^{n-1} e_{2}^{n-1}\left(e_{1}+e_{2}\right) \quad \text { or } \quad W_{0}^{\prime}=e_{1} e_{2}^{n-1}\left(e_{1}+e_{2}\right)^{n-1} .
$$

But in the second case, we would get $\sigma\left(W_{0}^{\prime}\right)=-2 e_{2} \neq 0$. Thus $W_{0}^{\prime}=$ $e_{1}^{n-1} e_{2}^{n-1}\left(e_{1}+e_{2}\right)$ and $\varphi\left(W_{i}\right)=e_{1}^{n}$.

CASE 1.2: $\mathrm{h}\left(\varphi\left(W_{0} W_{i}\right)\right)=n$. We distinguish two further subcases.

CASE 1.2.1: $\varphi\left(W_{i}\right)=g^{n}$ for some $g \in \varphi(G) \backslash\left\{e_{1}, e_{2}, e_{1}+e_{2}\right\}$. We set $g=c e_{1}+d e_{2}$ with $c, d \in[0, n-1]$. By Lemmas 2.2 and 2.5, it follows that $\varphi\left(W_{0}\right) g^{n-1}$ has a zero-sum subsequence $T$ of length $|T|=n$ and $\varphi\left(W_{i} W_{0}\right) T^{-1}$ is a minimal zero-sum subsequence of $\varphi(S)$ of length $2 n-1$, say

$$
\varphi\left(W_{i} W_{0}\right) T^{-1}=e_{2}^{q} e_{1}^{r}\left(e_{1}+e_{2}\right)^{s}\left(c e_{1}+d e_{2}\right)^{t},
$$

where $q \geq 1, r \geq 1, s \geq 0$ and $t \in[1, n-1]$.

Since $g \neq e_{1}+e_{2}$, we infer that $s \leq 1$. If $s=1$, then, by the assumption of Case 1, we get

$$
\begin{aligned}
2 n-1 & =\left|W_{i} W_{0} T^{-1}\right|=q+r+s+t \geq 1+(q+r+t) \\
& \geq 1+(n-1+n-1+1)>2 n-1,
\end{aligned}
$$

a contradiction. Hence $s=0$. Again, by the assumption of Case 1, we have the following possibilities:

- $q=r=n-1$ and $t=1$.

- $q=t=n-1$ and $r=1$.

- $q=1$ and $r=t=n-1$.

If $q=r=n-1$ and $t=1$, then $\sigma\left(\varphi\left(W_{0} W_{i}\right) T^{-1}\right)=0$ implies that $g=e_{1}+e_{2}$, a contradiction. If $q=t=n-1$ and $r=1$, then $\sigma\left(\left(W_{0} W_{i}\right) T^{-1}\right)=0$ implies that $g=e_{1}-e_{2}$ and $\varphi\left(W_{i}\right)=\left(e_{1}-e_{2}\right)^{n}$. Finally, if $q=1$ and $r=t=n-1$, then $\sigma\left(\varphi\left(W_{0} W_{i}\right) T^{-1}\right)=0$ implies that $g=-e_{1}+e_{2}$ and $\varphi\left(W_{i}\right)=\left(-e_{1}+e_{2}\right)^{n}$.

CASE 1.2.2: $\vee_{g}\left(\varphi\left(W_{0} W_{i}\right)\right)=n$ for some $g \in\left\{e_{1}, e_{2}, e_{1}+e_{2}\right\}$. Since $\left|W_{i}\right|$ $=n, \sigma\left(\varphi\left(W_{i}\right)\right)=0$ and $\mathrm{v}_{e_{1}+e_{2}}\left(\varphi\left(W_{0}\right)\right)=1$, it follows that $g \neq e_{1}+e_{2}$. Thus 
$g \in\left\{e_{1}, e_{2}\right\}$, say $g=e_{1}$. Then

$$
\varphi\left(W_{0} W_{i}\right)=e_{2}^{n-1}\left(e_{1}+e_{2}\right) e_{1}^{n} \prod_{\nu=1}^{n-1}\left(c_{\nu} e_{1}+d_{\nu} e_{2}\right),
$$

where $c_{\nu}, d_{\nu} \in[0, n-1]$ for all $\nu \in[1, n-1]$. By Lemma 2.5 and the assumption of Case 1.2,

$$
W_{0}^{\prime}=e_{2}^{n-1}\left(e_{1}+e_{2}\right) \prod_{\nu=1}^{n-1}\left(c_{\nu} e_{1}+d_{\nu} e_{2}\right)
$$

is a minimal zero-sum subsequence of $\varphi(S)$ with $e_{1} \nmid W_{0}^{\prime}$. Since $W_{0}^{\prime}$ contains two distinct elements with multiplicity $n-1$ (by the assumption of Case 1) and $\sigma\left(\varphi\left(W_{i}\right)\right)=0$, and since $e_{1} \nmid W_{0}^{\prime}$, it follows that

$$
W_{0}^{\prime}=e_{2}^{n-1}\left(e_{1}+e_{2}\right)^{n-1}\left(e_{1}+2 e_{2}\right),
$$

and thus

$$
\varphi\left(W_{i}\right)=e_{1}\left(e_{1}+e_{2}\right)^{n-2}\left(e_{1}+2 e_{2}\right) .
$$

CASE 1.3: $\mathrm{h}\left(\varphi\left(W_{0} W_{i}\right)\right)=n-1$. Since $\sigma\left(\varphi\left(W_{i}\right)\right)=0$, it follows from (2) that $\mathrm{v}_{g}\left(\varphi\left(W_{0} W_{i}\right)\right) \neq n-1$ for $g \notin\left\{e_{1}, e_{2}, e_{1}+e_{2}\right\}$. Suppose $\mathrm{v}_{e_{1}+e_{2}}\left(\varphi\left(W_{0} W_{i}\right)\right)$ $=n-1$. Then

$$
\varphi\left(W_{i}\right)=\left(e_{1}+e_{2}\right)^{n-2}\left(c_{1} e_{1}+d_{1} e_{1}\right)\left(c_{2} e_{1}+d_{2} e_{2}\right),
$$

where $c_{1}, d_{1}, c_{2}, d_{2} \in[0, n-1]$. By Lemma 2.2 and the definition of Property $\mathbf{C}$,

$$
\varphi\left(W_{0} W_{i}\right)\left(e_{1}+e_{2}\right)^{-1}\left(c_{2} e_{1}+d_{2} e_{2}\right)^{-1}
$$

has a zero-sum subsequence $T$ of length $|T|=n$ and, by Lemma 2.5 . $\varphi\left(W_{0} W_{i}\right) T^{-1}$ is a minimal zero-sum subsequence of $\varphi(S)$ of length $2 n-1$. In view of the assumptions of Case 1 and Case 1.3, and in view of

$$
\varphi\left(W_{0} W_{i}\right)=e_{1}^{n-1} e_{2}^{n-1}\left(e_{1}+e_{2}\right)^{n-1}\left(c_{1} e_{1}+d_{1} e_{2}\right)\left(c_{2} e_{1}+d_{2} e_{2}\right),
$$

it follows that $\mathrm{h}(T)=n-1$, contradicting $\sigma(T)=0$. So we conclude that

$$
\mathrm{v}_{g}\left(\varphi\left(W_{0} W_{i}\right)\right)<n-1 \quad \text { for all } g \in \varphi(G) \backslash\left\{e_{1}, e_{2}\right\} .
$$

We set $\varphi\left(W_{i}\right)=\prod_{\nu=1}^{n}\left(c_{\nu} e_{1}+d_{\nu} e_{2}\right)$, where $c_{\nu}, d_{\nu} \in[0, n-1]$ for all $\nu \in[1, n]$, and pick some $\lambda \in[1, n]$. By Lemmas 2.2 and 2.5, it follows that $\varphi\left(W_{0} W_{i}\right)\left(c_{\lambda} e_{1}+d_{\lambda} e_{2}\right)^{-1}$ has a zero-sum subsequence $T$ of length $|T|=n$ and that $\varphi\left(W_{i} W_{0}\right) T^{-1}$ is a minimal zero-sum subsequence of $\varphi(S)$ of length $2 n-1$. By the assumption of Case 1 and (3), it follows that

$$
\varphi\left(W_{0} W_{i}\right) T^{-1}=e_{1}^{n-1} e_{2}^{n-1}\left(e_{1}+e_{2}\right),
$$

and thus $c_{\lambda} e_{1}+d_{\lambda} e_{2}=e_{1}+e_{2}$. As $\lambda \in[1, n]$ was arbitrary, this implies that $\varphi\left(W_{i}\right)=\left(e_{1}+e_{2}\right)^{n}$, contradicting the hypothesis of Case 1.3. 
CASE 2: There exists a product decomposition $W \in \Omega$ with $\vee_{g}\left(\varphi\left(W_{0}\right)\right)=$ $n-1$ for exactly one element $g \in \varphi(G)$. By Lemma 2.3 . 1 and the assumption of Case 2, there exists a basis $\left(e_{1}, e_{2}\right)$ of $\varphi(G)$ such that

$$
\varphi\left(W_{0}\right)=e_{1}^{n-1} \prod_{\nu=1}^{n}\left(x_{\nu} e_{1}+e_{2}\right),
$$

where $x_{1}, \ldots, x_{n} \in[0, n-1]$ and $x_{1}+\cdots+x_{n} \equiv 1 \bmod n$ and at most $n-2$ of the elements $x_{1}, \ldots, x_{n}$ are equal. Let $i \in[1,2 m-2]$ be arbitrary, and let $\varphi\left(W_{i}\right)=\prod_{\nu=1}^{n}\left(c_{\nu} e_{1}+d_{\nu} e_{2}\right)$, where $c_{\nu}, d_{\nu} \in[0, n-1]$ for all $\nu \in[1, n]$. We proceed to show that there exists $m_{i} \in\{0, n\}$ such that

$$
\varphi\left(W_{i}\right)=e_{1}^{m_{i}} \prod_{\nu=1}^{n-m_{i}}\left(c_{\nu} e_{1}+e_{2}\right),
$$

which will complete the proof. Let $W_{i}=\prod_{\nu=1}^{n}\left(c_{\nu} e_{1}+d_{\nu} e_{2}\right)$. We distinguish six subcases.

CASE 2.1: $\mathrm{h}\left(\varphi\left(W_{i}\right) \prod_{\nu=1}^{n}\left(x_{\nu} e_{1}+e_{2}\right)\right)>n$. Then there exists some $x \in$ $[0, n-1]$ such that (after renumbering if necessary)

$$
\varphi\left(W_{i}\right) \prod_{\nu=1}^{n}\left(x_{\nu} e_{1}+e_{2}\right)=\left(x e_{1}+e_{2}\right)^{n} \prod_{\nu=1}^{r}\left(c_{\nu} e_{1}+d_{\nu} e_{2}\right) \prod_{\nu=1}^{s}\left(x_{\nu} e_{1}+e_{2}\right),
$$

where $r \in[1, n-1], s \in[2, n-1]$ and $r+s=n$. Since

$$
e_{1}^{n-1} \prod_{\nu=1}^{r}\left(c_{\nu} e_{1}+d_{\nu} e_{2}\right) \prod_{\nu=1}^{s}\left(x_{\nu} e_{1}+e_{2}\right)
$$

is a minimal zero-sum subsequence of $\varphi(S)$ of length $2 n-1$, Lemma 2.3 implies that $d_{1}=\cdots=d_{r}=1$, whence $\varphi\left(W_{i}\right)=\prod_{\nu=1}^{n}\left(c_{\nu} e_{1}+e_{2}\right)$.

CASE 2.2: $\mathrm{h}\left(\varphi\left(W_{i}\right) \prod_{\nu=1}^{n}\left(x_{\nu} e_{1}+e_{2}\right)\right)=n$. If $\left(c_{1}, d_{1}\right)=\cdots=\left(c_{n}, d_{n}\right)$ does not hold, then, similar to Case 2.1, we obtain $d_{1}=\cdots=d_{n}=1$. Therefore $c_{1}=\cdots=c_{n}=c$ and $d_{1}=\cdots=d_{n}=d$ for some $c, d \in[0, n-1]$.

Pick some $\lambda \in[1, n]$ and consider the sequence

$$
\begin{aligned}
\varphi\left(W_{0} W_{i}\right)\left(x_{\lambda} e_{1}+e_{2}\right)^{-1}\left(c e_{1}+d e_{2}\right)^{-1} & \\
= & \left(c e_{1}+d e_{2}\right)^{n-1} e_{1}^{n-1} \prod_{\nu \in[1, n] \backslash\{\lambda\}}\left(x_{\nu} e_{1}+e_{2}\right) .
\end{aligned}
$$

Since this sequence has length $3 n-3=\eta\left(C_{n} \oplus C_{n}\right)-1$ (by Lemma 2.2.1) but is not of the form $U^{n-1}$ with $U \in \mathcal{F}\left(C_{n} \oplus C_{n}\right)$ (by the assumption of Case 2), it follows from Lemma 2.2.2 and the definition of Property $\mathbf{C}$ that it has a zero-sum subsequence $T$ of length $n$. Moreover, by Lemma 2.5.1, $\varphi\left(W_{0} W_{1}\right) T^{-1}$ is a minimal zero-sum sequence of length $2 n-1$. Since $\varphi(G)$ 
has Property $\mathbf{B}$, we have either

$$
e_{1}^{n-1} \mid \varphi\left(W_{0} W_{i}\right) T^{-1} \quad \text { or } \quad\left(c e_{1}+d e_{2}\right)^{n-1} \mid \varphi\left(W_{0} W_{i}\right) T^{-1} .
$$

If $e_{1}^{n-1} \mid \varphi\left(W_{0} W_{i}\right) T^{-1}$, then, since $\left(x_{\lambda} e_{1}+e_{2}\right)\left(c e_{1}+d e_{2}\right) \mid \varphi\left(W_{0} W_{i}\right) T^{-1}$, it would follow that $d=1$, whence $\varphi\left(W_{i}\right)=\left(c e_{1}+e_{2}\right)^{n}$, as desired. Therefore we may assume that $\left(c e_{1}+d e_{2}\right)^{n-1} \mid \varphi\left(W_{0} W_{i}\right) T^{-1}$.

Since $\varphi\left(W_{i}\right)$ is a minimal zero-sum sequence, it follows that

$$
n=\operatorname{ord}\left(c e_{1}+d e_{2}\right)=n / \operatorname{gcd}(c, d, n),
$$

and hence there are $u, v \in \mathbb{Z}$ such that $u c+v d \equiv 1 \bmod n$. Thus

$$
\left(e_{1}^{\prime}, e_{2}^{\prime}\right)=\left(c e_{1}+d e_{2},-v e_{1}+u e_{2}\right)=\left(e_{1}, e_{2}\right) \cdot\left(\begin{array}{cc}
c & -v \\
d & u
\end{array}\right)
$$

is a basis of $\varphi(G)$ and, for some sequence $Q$ over $\varphi(G)$,

$$
\begin{aligned}
\varphi\left(W_{0} W_{i}\right) T^{-1} & =\left(c e_{1}+d e_{2}\right)^{n-1} e_{1}\left(x_{\lambda} e_{1}+e_{2}\right) Q \\
& =e_{1}^{\prime n-1}\left(u e_{1}^{\prime}-d e_{2}^{\prime}\right)\left(\left(x_{\lambda} u+v\right) e_{1}^{\prime}+\left(c-x_{\lambda} d\right) e_{2}^{\prime}\right) Q .
\end{aligned}
$$

Now Lemma 2.3 implies that $-d \equiv c-x_{\lambda} d \bmod n$, whence $x_{\lambda} d \equiv c+d \bmod n$. Therefore, since $\lambda$ was arbitrary, we get

$$
d \equiv \sum_{\nu=1}^{n} x_{\nu} d \equiv n(c+d) \equiv 0 \bmod n,
$$

and thus $d=0$. If $c \in[2, n]$, then $\left(c e_{1}\right) e_{1}^{n-c}$ is a zero-sum subsequence of $\varphi(S)$ of length $n-c+1<n$, a contradiction. Thus $c=1$ and $\varphi\left(W_{i}\right)=e_{1}^{n}$.

CASE 2.3: $\mathrm{h}\left(\varphi\left(W_{i}\right) \prod_{\nu=1}^{n}\left(x_{\nu} e_{1}+e_{2}\right)\right)=n-1$ and $\mathrm{v}_{e_{1}}\left(\varphi\left(W_{i}\right)\right) \geq 2$. After renumbering if necessary, we have

$$
\varphi\left(W_{0} W_{i}\right)=e_{1}^{n+1}\left(x e_{1}+e_{2}\right)^{n-1} \prod_{\nu=1}^{r}\left(x_{\nu} e_{1}+e_{2}\right) \prod_{\nu=1}^{s}\left(c_{\nu} e_{1}+d_{\nu} e_{2}\right)
$$

where $x \in[0, n-1], r \in[1, n-1], s \in[1, n-2]$ and $r+s=n-1$. By Lemma 2.5,

$$
W^{\prime}=e_{1}\left(x e_{1}+e_{2}\right)^{n-1} \prod_{\nu=1}^{r}\left(x_{\nu} e_{1}+e_{2}\right) \prod_{\nu=1}^{s}\left(c_{\nu} e_{1}+d_{\nu} e_{2}\right)
$$

is a minimal zero-sum subsequence of $\varphi(S)$ of length $2 n-1$. Since

$$
\left(e_{1}, e_{2}^{\prime}\right)=\left(e_{1}, x e_{1}+e_{2}\right)=\left(e_{1}, e_{2}\right) \cdot\left(\begin{array}{ll}
1 & x \\
0 & 1
\end{array}\right)
$$

is a basis of $\varphi(G)$ and

$$
W^{\prime}=e_{1} e_{2}^{\prime n-1} \prod_{\nu=1}^{r}\left(\left(x_{\nu}-x\right) e_{1}+e_{2}^{\prime}\right) \prod_{\nu=1}^{s}\left(\left(c_{\nu}-x d_{\nu}\right) e_{1}+d_{\nu} e_{2}^{\prime}\right)
$$


Lemma 2.3.2 implies that $x_{\nu}-x \equiv 1 \bmod n$ for all $\nu \in[1, r]$. Therefore, since

$$
\varphi\left(W_{0}\right)=e_{1}^{n-1}\left(x e_{1}+e_{2}\right)^{n-r} \prod_{\nu=1}^{r}\left(x_{\nu} e_{1}+e_{2}\right)
$$

(in view of (4)), we get $(n-r) x+r(x+1) \equiv \sum_{\nu=1}^{n} x_{\nu} \equiv 1 \bmod n$. Hence $r=1$ and

$$
\varphi\left(W_{0}\right)=e_{1}^{n-1}\left(x e_{1}+e_{2}\right)^{n-1}\left((x+1) e_{1}+e_{2}\right),
$$

a contradiction to our assumption on $x_{1}, \ldots, x_{n}$ for Case 2 .

CASE 2.4: $\mathrm{h}\left(\varphi\left(W_{i}\right) \prod_{\nu=1}^{n}\left(x_{\nu} e_{1}+e_{2}\right)\right)=n-1$ and $v_{e_{1}}\left(W_{i}\right)=1$. After renumbering if necessary, we get

$$
\varphi\left(W_{0} W_{i}\right)=e_{1}^{n}\left(x e_{1}+e_{2}\right)^{n-1} \prod_{\nu=1}^{r}\left(x_{\nu} e_{1}+e_{2}\right) \prod_{\nu=1}^{s}\left(c_{\nu} e_{1}+d_{\nu} e_{2}\right)
$$

with $x \in[0, n-1], r \in[1, n-1], s \in[1, n-1]$ and $r+s=n$. By Lemma 2.5.

$$
W^{\prime}=\left(x e_{1}+e_{2}\right)^{n-1} \prod_{\nu=1}^{r}\left(x_{\nu} e_{1}+e_{2}\right) \prod_{\nu=1}^{s}\left(c_{\nu} e_{1}+d_{\nu} e_{2}\right)
$$

is a minimal zero-sum subsequence of $\varphi(S)$ of length $2 n-1$. Since

$$
\left(e_{1}, e_{2}^{\prime}\right)=\left(e_{1}, x e_{1}+e_{2}\right)=\left(e_{1}, e_{2}\right) \cdot\left(\begin{array}{ll}
1 & x \\
0 & 1
\end{array}\right)
$$

is a basis of $\varphi(G)$ and

$$
W^{\prime}=e_{2}^{\prime n-1} \prod_{\nu=1}^{r}\left(\left(x_{\nu}-x\right) e_{1}+e_{2}^{\prime}\right) \prod_{\nu=1}^{s}\left(\left(c_{\nu}-x d_{\nu}\right) e_{1}+d_{\nu} e_{2}^{\prime}\right),
$$

Lemma 2.3.2 implies that

$$
x_{1}-x \equiv \cdots \equiv x_{r}-x \equiv c_{1}-x d_{1} \equiv \cdots \equiv c_{s}-x d_{s} \bmod n .
$$

If $d_{1}=\cdots=d_{s}=1$, then $\varphi\left(W_{i}\right)=\prod_{\nu=1}^{n}\left(c_{\nu} e_{1}+e_{2}\right)$, as desired. Therefore we may assume there is some $\nu \in[1, s]$ with $d_{\nu} \neq 1$, say $\nu=s$. Hence, since $\sigma\left(W_{i}\right)=0$, it follows that there is also another $\nu^{\prime} \in[1, s]$ with $d_{\nu^{\prime}} \neq 1$ and $s=\nu \neq \nu^{\prime}$. Thus, by Lemmas 2.2 and 2.5 and the definition of Property $\mathbf{C}$,

$$
\varphi\left(W_{0} W_{i}\right) e_{1}^{-1}\left(c_{s} e_{1}+d_{s} e_{2}\right)^{-1}
$$

has a zero-sum subsequence $T$ of length $|T|=n$ and $\varphi\left(W_{0} W_{i}\right) T^{-1}$ is a minimal zero-sum subsequence of $\varphi(S)$ of length $2 n-1$. Since $\varphi(G)$ has Property $\mathbf{B}$, it follows that either

$$
e_{1}^{n-1} \mid \varphi\left(W_{0} W_{i}\right) T^{-1} \quad \text { or } \quad\left(x e_{1}+e_{2}\right)^{n-1} \mid \varphi\left(W_{0} W_{i}\right) T^{-1} .
$$

If $e_{1}^{n-1} \mid \varphi\left(W_{0} W_{i}\right) T^{-1}$, then, as $\left(c_{s} e_{1}+d_{s} e_{2}\right) \mid \varphi\left(W_{0} W_{i}\right) T^{-1}$ and $\left(x_{j} e_{1}+e_{2}\right) \mid$ $\varphi\left(W_{0} W_{i}\right) T^{-1}$ for some $j \in[1, n]$, Lemma 2.3 implies that $d_{s}=1$, a contra- 
diction. Therefore $\left(x e_{1}+e_{2}\right)^{n-1} \mid \varphi\left(W_{0} W_{i}\right) T^{-1}$. Thus, for some sequence $Q$ over $\varphi(G)$, we have

$$
\varphi\left(W_{0} W_{i}\right) T^{-1}=\left(x e_{1}+e_{2}\right)^{n-1} e_{1}\left(c_{s} e_{1}+d_{s} e_{2}\right) Q .
$$

Since

$$
\left(e_{1}, e_{2}^{\prime}\right)=\left(e_{1}, x e_{1}+e_{2}\right)=\left(e_{1}, e_{2}\right) \cdot\left(\begin{array}{ll}
1 & x \\
0 & 1
\end{array}\right)
$$

is a basis of $\varphi(G)$ and

$$
\varphi\left(W_{0} W_{i}\right) T^{-1}=e_{1} e_{2}^{\prime n-1}\left(\left(c_{s}-x d_{s}\right) e_{1}+d_{s} e_{2}^{\prime}\right) Q,
$$

Lemma 2.3 implies that $c_{s}-x d_{s}=1$. Thus it follows from (5) that $x_{1} \equiv \cdots \equiv$ $x_{r} \equiv x+1 \bmod n$. Therefore we get $(n-r) x+r(x+1) \equiv \sum_{\nu=1}^{n} x_{\nu} \equiv 1 \bmod n$. Hence $r=1$ and

$$
\varphi\left(W_{0}\right)=e_{1}^{n-1}\left(x e_{1}+e_{2}\right)^{n-1}\left((x+1) e_{1}+e_{2}\right),
$$

a contradiction to our assumption on $x_{1}, \ldots, x_{n}$ for Case 2 .

CASE 2.5: $\mathrm{h}\left(\varphi\left(W_{i}\right) \prod_{\nu=1}^{n}\left(x_{\nu} e_{1}+e_{2}\right)\right)=n-1$ and $\mathrm{v}_{e_{1}}\left(\varphi\left(W_{i}\right)\right)=0$. If $d_{1}=\cdots=d_{n}=1$, then the assertion follows. Therefore there is some $\nu \in[1, n]$ with $d_{\nu} \neq 1$, say $\nu=n$. Since $d_{1}+\cdots+d_{n} \equiv 0 \bmod n$, we may also assume that $d_{n-1} \neq 1$. We distinguish two subcases.

CASE 2.5.1: $\varphi\left(W_{i}\right) \prod_{\nu=1}^{n}\left(x_{\nu} e_{1}+e_{2}\right)$ contains two distinct elements with multiplicity $n-1$, say $x e_{1}+e_{2}$ and $y e_{1}+e_{2}$, where $x, y \in[0, n-1]$. Then

$$
\varphi\left(W_{i}\right)=\left(x e_{1}+e_{2}\right)^{r}\left(y e_{1}+e_{2}\right)^{s}\left(c_{n-1} e_{1}+d_{n-1} e_{2}\right)\left(c_{n} e_{1}+d_{n} e_{2}\right)
$$

and

$$
\prod_{\nu=1}^{n}\left(x_{\nu} e_{1}+e_{2}\right)=\left(x e_{1}+e_{2}\right)^{n-1-r}\left(y e_{1}+e_{2}\right)^{n-1-s},
$$

where $r, s \in[1, n-3]$ and $r+s=n-2 \geq 2$. By Lemmas 2.2 and 2.5 . $\varphi\left(W_{0} W_{i}\right)\left(c_{n} e_{1}+d_{n} e_{2}\right)^{-1}$ has a zero-sum subsequence $T$ of length $|T|=n$ and $\varphi\left(W_{0} W_{i}\right) T^{-1}$ is a minimal zero-sum subsequence of $\varphi(S)$ of length $2 n-1$. Since $\varphi(G)$ has Property $\mathbf{B}$, it follows that

$$
\mathrm{v}_{g}\left(\varphi\left(W_{i} W_{0}\right) T^{-1}\right)=n-1 \quad \text { for some } g \in\left\{e_{1}, x e_{1}+e_{2}, y e_{1}+e_{2}\right\} .
$$

Clearly, we have

$$
e_{1}\left(x e_{1}+e_{2}\right)\left(y e_{1}+e_{2}\right)\left(c_{n} e_{1}+d_{n} e_{2}\right) \mid \varphi\left(W_{0} W_{i}\right) T^{-1} .
$$

Since $d_{n} \neq 1$, Lemma 2.3 implies that $g \neq e_{1}$. Thus w.l.o.g. $g=x e_{1}+e_{2}$. Consequently, for some sequence $Q$ over $\varphi(G)$, we have

$$
\varphi\left(W_{0} W_{i}\right) T^{-1}=\left(x e_{1}+e_{2}\right)^{n-1} e_{1}\left(y e_{1}+e_{2}\right) Q .
$$


As before,

$$
\left(e_{1}, e_{2}^{\prime}\right)=\left(e_{1}, x e_{1}+e_{2}\right)=\left(e_{1}, e_{2}\right) \cdot\left(\begin{array}{ll}
1 & x \\
0 & 1
\end{array}\right)
$$

is a basis of $\varphi(G)$ and

$$
\varphi\left(W_{0} W_{i}\right) T^{-1}=e_{2}^{\prime n-1} e_{1}\left((y-x) e_{1}+e_{2}^{\prime}\right) Q .
$$

Now Lemma 2.3.2 implies $y-x \equiv 1 \bmod n$, whence (6) and $\sum_{\nu=1}^{n} x_{\nu} \equiv$ $1 \bmod n$ imply $\left(x e_{1}+e_{2}\right)^{n-1} \mid W_{0}$, contradicting the assumption of Case 2.

CASE 2.5.2: $\varphi\left(W_{i}\right) \prod_{\nu=1}^{n}\left(x_{\nu} e_{1}+e_{2}\right)$ contains exactly one element with multiplicity $n-1$, say $x e_{1}+e_{2}$ where $x \in[0, n-1]$. After renumbering if necessary, we get

$$
\varphi\left(W_{0} W_{i}\right)=e_{1}^{n-1}\left(x e_{1}+e_{2}\right)^{n-1} \prod_{\nu=1}^{r}\left(c_{\nu} e_{1}+d_{\nu} e_{2}\right) \prod_{\nu=1}^{s}\left(x_{\nu} e_{1}+e_{2}\right),
$$

where $r \in[1, n-1], s \in[2, n-1]$ and $r+s=n+1$. If $d_{1}=\cdots=d_{r}=1$, then the assertion follows. So after renumbering again, we suppose that $d_{r} \neq 1$. Let $\lambda \in[1, s]$.

By Lemmas 2.2 and 2.5, the definition of Property $\mathbf{C}$, and the assumption of Case 2.5.2,

$$
\varphi\left(W_{0} W_{i}\right)\left(c_{r} e_{1}+d_{r} e_{2}\right)^{-1}\left(x_{\lambda} e_{1}+e_{2}\right)^{-1}
$$

has a zero-sum subsequence $T$ of length $|T|=n$ and $\varphi\left(W_{0} W_{i}\right) T^{-1}$ is a minimal zero-sum subsequence of $\varphi(S)$ of length $2 n-1$. Since $\varphi(G)$ has Property B, it follows that

$$
\mathrm{v}_{g}\left(\varphi\left(W_{0} W_{i}\right) T^{-1}\right)=n-1 \quad \text { for some } g \in\left\{e_{1}, x e_{1}+e_{2}\right\} .
$$

Clearly, we have

$$
e_{1}\left(x e_{1}+e_{2}\right)\left(c_{r} e_{1}+d_{r} e_{2}\right)\left(x_{\lambda} e_{1}+e_{2}\right) \mid \varphi\left(W_{0} W_{i}\right) T^{-1} .
$$

Since $d_{r} \neq 1$, Lemma 2.3 implies that $g \neq e_{1}$, and hence $g=x e_{1}+e_{2}$. Thus, for some sequence $Q$ over $\varphi(G)$, we have

$$
\varphi\left(W_{0} W_{i}\right) T^{-1}=\left(x e_{1}+e_{2}\right)^{n-1} e_{1}\left(x_{\lambda} e_{1}+e_{2}\right) Q .
$$

As before,

$$
\left(e_{1}, e_{2}^{\prime}\right)=\left(e_{1}, x e_{1}+e_{2}\right)=\left(e_{1}, e_{2}\right) \cdot\left(\begin{array}{ll}
1 & x \\
0 & 1
\end{array}\right)
$$

is a basis of $\varphi(G)$ and

$$
\varphi\left(W_{0} W_{i}\right) T^{-1}=e_{2}^{\prime n-1} e_{1}\left(\left(x_{\lambda}-x\right) e_{1}+e_{2}^{\prime}\right) Q .
$$

Hence Lemma 2.3 implies that $1 \equiv x_{\lambda}-x \bmod n$. As $\lambda \in[1, s]$ was arbitrary, it follows that $x_{1} \equiv \cdots \equiv x_{s} \equiv x+1 \bmod n$, and, as in Case 2.3, we obtain a contradiction. 
CASE 2.6: $\mathrm{h}\left(\varphi\left(W_{i}\right) \prod_{\nu=1}^{n}\left(x_{\nu} e_{1}+e_{2}\right)\right)<n-1$. Let $\lambda \in[1, n]$ be arbitrary. By Lemmas 2.2 and 2.5. $\varphi\left(W_{0} W_{i}\right)\left(c_{\lambda} e_{1}+d_{\lambda} e_{2}\right)^{-1}$ has a zero-sum subsequence $T$ of length $|T|=n$, and $\varphi\left(W_{0} W_{i}\right) T^{-1}$ is a minimal zero-sum subsequence of $\varphi(S)$ of length $2 n-1$. Since $\varphi(G)$ has Property B, it follows that $e_{1}^{n-1}$ divides $\varphi\left(W_{0} W_{i}\right) T^{-1}$. Furthermore, there is some $\nu \in[1, n]$ such that

$$
\left(x_{\nu} e_{1}+e_{2}\right)\left(c_{\lambda} e_{1}+d_{\lambda} e_{2}\right) \mid \varphi\left(W_{0} W_{i}\right) T^{-1} .
$$

Thus Lemma 2.5 implies that either $d_{\lambda}=1$ or $\left(c_{\lambda}, d_{\lambda}\right)=(1,0)$. Thus, since $\lambda \in[1, n]$ was arbitrary and $\sigma\left(\varphi\left(W_{i}\right)\right)=0$, we must have either $d_{\lambda}=1$ for all $\lambda \in[1, n]$, or $\left(c_{\lambda}, d_{\lambda}\right)=(1,0)$ for all $\lambda \in[1, n]$, and so either $\varphi\left(W_{i}\right)=e_{1}^{n}$ or $\varphi\left(W_{i}\right)=\prod_{\nu=1}^{n}\left(c_{\nu} e_{1}+e_{2}\right)$, as desired.

5. Proof of the Theorem. Let $G=C_{m n} \oplus C_{m n}$, with $m, n \geq 3$ odd, be such that Property $\mathbf{B}$ holds for both $C_{m} \oplus C_{m}$ and $C_{n} \oplus C_{n}$ (if $m=1$ or $n=1$, then the Theorem is trivial). Since Property $\mathbf{B}$ holds when $m n=9$ (as shown in [2] and mentioned in the introduction), we may assume $m n>9$, and w.l.o.g. $m \geq 5$. Let $S \in \mathcal{A}(G)$ be a minimal zero-sum sequence of length $|S|=2 m n-1$. The sequence $S$ will remain fixed throughout the rest of this section. Our goal is to show that $S$ contains an element with multiplicity $m n-1$ (in other words, $\mathrm{h}(S)=m n-1$ ). We proceed in the following way:

- First, using Proposition 4.2, we establish the setting and some detailed notation necessary to formulate the key ideas of the proof.

- Next, we proceed with four lemmas, 5.1 5.4, that collect several arguments used repeatedly in the proof.

- Then, we divide the main part of the proof into four claims, A, B, C and $\mathrm{D}$, where in Claim D we finally show that $\mathrm{h}(S)=m n-1$.

The setting and key definitions. Since $S$ is fixed, we write $\Omega^{\prime}$ and $\Omega$ instead of $\Omega^{\prime}(S)$ and $\Omega(S)$ (see Definition 4.1). Recall that Lemma 2.3 .3 implies that $\operatorname{ord}(x)=m n$ for all $x \in \operatorname{supp}(S)$. Let $\varphi: G \rightarrow G$ denote multiplication by $m$. Then $\operatorname{Ker}(\varphi)=n G \cong C_{m} \oplus C_{m}$ and $\varphi(G)=m G \cong$ $C_{n} \oplus C_{n}$.

Let $\Omega_{0} \subset \Omega$ be all those $W \in \Omega$ for which there exists a basis $\left(m e_{1}, m e_{2}\right)$ of $\varphi(G)$, where $e_{1}, e_{2} \in G$, such that $\varphi\left(W_{0}\right)=\left(m e_{1}\right)^{n-1} \prod_{\nu=1}^{n}\left(x_{\nu} m e_{1}+m e_{2}\right)$, where $x_{1}, \ldots, x_{n} \in \mathbb{Z}$ with $x_{1}+\cdots+x_{n} \equiv 1 \bmod n$, and such that for every $i \in[1,2 m-2], \varphi\left(W_{i}\right)$ is of the form either $\varphi\left(W_{i}\right)=\left(m e_{1}\right)^{n}$, or $\varphi\left(W_{i}\right)=$ $\prod_{\nu=1}^{n}\left(y_{i, \nu} m e_{1}+m e_{2}\right)$ where $y_{i, 1}, \ldots, y_{i, n} \in \mathbb{Z}$ with $y_{i, 1}+\cdots+y_{i, n} \equiv 0 \bmod n$. By Proposition 4.2, $\Omega_{0}$ is nonempty.

Let $W \in \Omega^{\prime}$, and define $\widetilde{\sigma}(W)=\prod_{\nu=0}^{2 m-2} \sigma\left(W_{\nu}\right) \in \mathcal{F}(\operatorname{Ker}(\varphi))$. Since $S \in \mathcal{A}(G)$, it follows that $\tilde{\sigma}(W) \in \mathcal{A}(\operatorname{Ker}(\varphi))$. As Property B holds for $\operatorname{Ker}(\varphi)$, it follows that $\tilde{\sigma}(W) \in \Upsilon(\operatorname{Ker}(\varphi))$. Partition $\Omega_{0}=\Omega_{0}^{u} \cup \Omega_{0}^{n u}$ by 
letting $\Omega_{0}^{u}$ be those $W \in \Omega_{0}$ with $\widetilde{\sigma}(W) \in \Upsilon_{u}(\operatorname{Ker}(\varphi))$, and letting $\Omega_{0}^{n u}$ be those $W \in \Omega_{0}$ with $\widetilde{\sigma}(W) \in \Upsilon_{n u}(\operatorname{Ker}(\varphi))$.

Let $W \in \Omega_{0}$, let $\left(m e_{1}, m e_{2}\right)$ be a basis of $\varphi(G)$ as in the definition of $\Omega_{0}$ with $e_{1}, e_{2} \in G$, and let $\left(f_{1}, f_{2}\right)$ be a basis for $\operatorname{Ker}(\varphi)$ such that $\widetilde{\sigma}(W)$ can be written as in the definition of $\Upsilon(\operatorname{Ker}(\varphi))$. While we will at times change these bases, the value $m e_{1}$ will remain constant throughout the proof, and we only ever deal with $W^{\prime} \in \Omega_{0}$ having an associated basis $\left(m e_{1}^{\prime}, m e_{2}^{\prime}\right)$ if $m e_{1}^{\prime}=m e_{1}$ (one may redefine $\Omega_{0}$ to be the subcollection of product decompositions $W^{\prime}$ for which this is true).

Let $S_{1}$ be the subsequence of $S$ consisting of all terms $x$ with $\varphi(x)=$ $m e_{1}$, and define $S_{2}$ by $S=S_{1} S_{2}$. In view of the comments in the previous paragraph, both $S_{1}$ and $S_{2}$ (which depend upon $m e_{1}$ ) will remain constant throughout the proof. Let $I \subset \mathbb{Z}$ be an interval of length $n$. Each term $x$ of $S_{1}$ has a unique representation of the form $x=e_{1}+n g$ with $n g \in \operatorname{Ker}(\varphi)$ (where $g \in G$ ), and each term $x$ of $S_{2}$ has a unique representation of the form $x=a e_{1}+e_{2}+n g$ with $a \in I$ and $n g \in \operatorname{Ker}(\varphi)$ (where $g \in G$ ). Define $\psi(x)=n g \in \operatorname{Ker}(\varphi)$ and, for $x \in \operatorname{supp}\left(S_{2}\right)$, define $\iota(x)=a \in I \subset \mathbb{Z}$. Thus

$$
\begin{aligned}
x=e_{1}+\psi(x) & \text { for } x \mid S_{1}, \\
x=\iota(x) e_{1}+e_{2}+\psi(x) & \text { for } x \mid S_{2},
\end{aligned}
$$

where $\psi(x) \in \operatorname{Ker}(\varphi)=\left\langle f_{1}, f_{2}\right\rangle$ and $\iota(x) \in I$. We set

$$
\psi(x)=\psi_{1}(x)+\psi_{2}(x) \text {, where } \quad \psi_{1}(x) \in\left\langle f_{1}\right\rangle \text { and } \psi_{2}(x) \in\left\langle f_{2}\right\rangle .
$$

If $y \in \operatorname{Ker}(\varphi)$ with $y=y_{1} f_{1}+y_{2} f_{2}$, then we also use $\psi_{i}(y)$ to denote $y_{i} f_{i}$.

Note that, for $x \in \operatorname{supp}\left(S_{1}\right)$, the value of $\psi(x)$ depends upon the choice of $\left(e_{1}, e_{2}\right)$, and that, for $x \in \operatorname{supp}\left(S_{2}\right)$, the values of $\psi(x)$ and $\iota(x)$ depend upon the choice of $\left(e_{1}, e_{2}\right)$ and $I$. We will frequently need to vary the underlying choices for $\left(e_{1}, e_{2}\right)$ and $I$, and each time we do so the corresponding values of $\psi$ and $\iota$ will be affected. All maps will be extended to sequences as explained before Definition 2.1.

Let $\mathcal{A}_{1}(W)$ be those $W_{i}$ with either $i=0$ or $\varphi\left(W_{i}\right)=\left(m e_{1}\right)^{n}$, let $\mathcal{A}_{2}(W)$ be all remaining $W_{i}$ as well as $W_{0}$, and let $\mathcal{A}_{i}^{*}(W)=\mathcal{A}_{i}(W) \backslash\left\{W_{0}\right\}$ for $i \in\{1,2\}$. If $W \in \Omega_{0}^{u}$, let $\mathcal{C}_{0}(W)$ be all those $W_{i}$ with $\vee_{\sigma\left(W_{i}\right)}(\widetilde{\sigma}(W))<m-1$, let $\mathcal{C}_{1}(W)$ be all remaining $W_{i}$, and let $\mathcal{C}_{i}^{*}(W)=\mathcal{C}_{i}(W) \backslash\left\{W_{0}\right\}$ for $i \in\{0,1\}$. If $W \in \Omega_{0}^{n u}$, let $\mathcal{C}_{0}(W)$ be the unique $W_{i}$ with $\vee_{\sigma\left(W_{i}\right)}(\widetilde{\sigma}(W))<m-1$, and divide the remaining $2 m-2$ blocks $W_{i}$ into either $\mathcal{C}_{1}(W)$ or $\mathcal{C}_{2}(W)$ depending on the value of $\sigma\left(W_{i}\right)$; analogously define $\mathcal{C}_{i}^{*}(W)$ for $i \in\{0,1,2\}$. When the context is clear, the $W$ will be omitted from the notation. We regard the elements $W_{i}, W_{j} \in \mathcal{A}_{1}$ as distinct when $i \neq j$, follow the same convention for all other similar collections of $W_{i}$, and will refer to them as blocks.

Note that a starred collection of blocks simply refers to the fact that the block $W_{0}$ has been removed from the collection. We suggest that the reader 
Table 1. Notation

\begin{tabular}{|c|l|}
\hline $\mathcal{A}_{1}$ & $\begin{array}{l}\text { Blocks } W_{i} \text { with }\left(m e_{1}\right)^{n-1} \mid \varphi\left(W_{i}\right), \text { so } \varphi\left(W_{i}\right)=\left(m e_{1}\right)^{n} \\
\text { or } \varphi\left(W_{i}\right)=\varphi\left(W_{0}\right)=\left(m e_{1}\right)^{n-1} \prod_{\nu=1}^{n}\left(x_{\nu} m e_{1}+m e_{2}\right)\end{array}$ \\
\hline $\mathcal{A}_{2}$ & Blocks $W_{i}$ with $\varphi\left(W_{i}\right)=\prod_{\nu=1}^{n}\left(x_{\nu} m e_{1}+m e_{2}\right)$ or $W_{i}=W_{0}$ \\
\hline $\mathcal{C}_{1}, \mathcal{C}_{2}$ & $\begin{array}{l}\text { "Majority" blocks, i.e., blocks having as sum the same multiplicity } m-1 \\
\text { element; we usually choose the basis }\left(f_{1}, f_{2}\right) \text { so that the following descrip- } \\
\text { tions of the } \mathcal{C}_{i} \text { hold }\end{array}$ \\
\hline $\mathcal{C}_{1}$ & Blocks $W_{i}$ with $\sigma\left(W_{i}\right)=f_{1}$ \\
\hline $\mathcal{C}_{2}$ & Blocks $W_{i}$ with $\sigma\left(W_{i}\right)=f_{2}\left(\right.$ only applicable if $\left.W \in \Omega_{0}^{n u}\right)$ \\
\hline $\mathcal{C}_{0}$ & $\begin{array}{l}\text { "Minority" blocks } W_{i}, \text { having } \sigma\left(W_{i}\right)=C f_{1}+f_{2} \text { for some } C \in \mathbb{Z} ; \text { if } \\
W \in \Omega_{0}^{n u}, \text { then our usual basis choice implies } \mathcal{C}_{0}=\left\{W_{k}\right\} \text { with } \sigma\left(W_{k}\right)= \\
f_{1}+f_{2}\end{array}$ \\
\hline
\end{tabular}

keep a sketch, at any given moment of the proof, of the current assumptions and information known for each $\left|\mathcal{A}_{i} \cap \mathcal{C}_{j}\right|, i \in[1,2]$ and $j \in[0,2]$, as well as which $\mathcal{C}_{j}$ contains $W_{0}$, as this will prevent much confusion. Table 1 may also be useful to quickly recall the definitions of the $\mathcal{A}_{i}$ and $\mathcal{C}_{j}$.

We further subdivide $W_{0}=W_{0}^{(1)} W_{0}^{(2)}$ with $W_{0}^{(1)}=\operatorname{gcd}\left(W_{0}, S_{1}\right)$ and $W_{0}^{(2)}=\operatorname{gcd}\left(W_{0}, S_{2}\right)$, and for a pair of subsequences $X$ and $Y$ with $X Y \mid S_{2}$, we define $\epsilon^{\prime}(X, Y)$ to be the integer in $[1, n]$ congruent to $\sigma(\iota(X))-\sigma(\iota(Y))$ modulo $n$, and define $\epsilon(X, Y)$ to be the integer such that

$$
n-\epsilon^{\prime}(X, Y)+\sigma(\iota(X))-\sigma(\iota(Y))=\epsilon(X, Y) n .
$$

The main idea of the proof is to swap individual terms contained in the blocks of $W \in \Omega_{0}$ so as to keep the resulting product decomposition in $\Omega^{\prime}$. Using the lemmas from Section 3 , we will then derive information about the possible values of $\psi$ and $\iota$ on the terms that have been swapped. The next three paragraphs detail the three major types of swaps that we will use.

If $U, V \in \mathcal{A}_{1}$ are distinct (thus $U=W_{i}$ and $V=W_{j}$ for some $i$ and $j$ distinct), then we may exchange any subsequence $X \mid U$ for a subsequence $Y \mid V$ with $|Y|=|X|$ (if $U=W_{0}$, then $X$ must additionally lie within $W_{0}^{(1)}$, and likewise for $V$ ) and the resulting product decomposition $W^{\prime}$ will still lie in $\Omega_{0}$, equal to $W$ except that the blocks $U$ and $V$ of $W$ have been replaced by the blocks $U^{\prime}:=X^{-1} U Y$ and $V^{\prime}:=Y^{-1} V X$. Moreover,

$$
\sigma\left(V^{\prime}\right)=\sigma(V)+\sigma(\psi(X))-\sigma(\psi(Y)) .
$$

We refer to this as a type I swap.

If $V \in \mathcal{A}_{2}^{*}$, and $Y \mid V$ and $X \mid W_{0}^{(2)}$ are subsequences with $|X|=|Y|$, then by exchanging the sequence $Y \mid V$ for the sequence $R X \mid W_{0}$, where $R \mid W_{0}^{(1)}$ is any subsequence with $|R|=n-\epsilon^{\prime}(X, Y)$, we obtain a product 
decomposition $W^{\prime}$ that still lies in $\Omega^{\prime}$, equal to $W$ except that the blocks $V$ and $W_{0}$ of $W$ have been replaced by the blocks $V^{\prime}:=Y^{-1} V X R$ and $W_{0}^{\prime}:=R^{-1} X^{-1} W_{0} Y$. Moreover,

$$
\sigma\left(V^{\prime}\right)=\sigma(V)+\epsilon(X, Y) n e_{1}+\sigma(\psi(X))-\sigma(\psi(Y))+\sigma(\psi(R)) .
$$

We refer to this as a type II swap.

If $U, V \in \mathcal{A}_{2}$ are distinct, then we may exchange any subsequence $X \mid U$ for a subsequence $Y \mid V$ with $|Y|=|X|$ and $\sigma(\iota(X))=\sigma(\iota(Y)$ ) (and if $U=W_{0}$, then $X$ must additionally lie within $W_{0}^{(2)}$, and likewise for $V$ ) and the resulting product decomposition $W^{\prime}$ will still lie in $\Omega_{0}$, equal to $W$ except that the blocks $U$ and $V$ of $W$ have been replaced by the blocks $U^{\prime}:=X^{-1} U Y$ and $V^{\prime}:=Y^{-1} V X$. Moreover,

$$
\sigma\left(V^{\prime}\right)=\sigma(V)+\sigma(\psi(X))-\sigma(\psi(Y)) .
$$

We refer to this as a type III swap.

We will also often have to change from $W \in \Omega_{0}$ to another $W^{\prime} \in \Omega_{0}$. One common way to do this will be to find $U \in \mathcal{A}_{2}^{*}$ and $X \mid U W_{0}^{(2)}$ ( $X$ will often be a single element dividing $U)$. Then $\left|X^{-1} U W_{0}^{(2)}\right|=2 n-|X|$. If there is an $n$-term subsequence $U^{\prime} \mid X^{-1} U W_{0}^{(2)}$ with $\sigma\left(U^{\prime}\right) \in \operatorname{Ker}(\varphi)$ (as is guaranteed, in the case $|X|=1$, by Theorem 2.6.1 applied to $\varphi\left(x^{-1} U W_{0}^{(2)}\right)$ modulo $m e_{2}$; note the $m e_{2}$ coordinate of every term dividing $\varphi\left(x^{-1} U W_{0}^{(2)}\right)$ is constant, so any $n$-term subsequence of $\varphi\left(x^{-1} U W_{0}^{(2)}\right)$ with sum zero modulo $m e_{2}$ will be itself zero-sum), then, defining $W_{0}^{\prime}$ by $W_{0}^{\prime} U^{\prime}=W_{0} U$, we obtain a new product decomposition $W^{\prime} \in \Omega_{0}$ by replacing the blocks $W_{0}$ and $U$ by $W_{0}^{\prime}$ and $U^{\prime}$. Moreover, $X \mid W_{0}^{\prime(2)}$. We refer to such a procedure as pulling $X$ up into the new product decomposition $W^{\prime}$.

All of the above procedures result in a new product decomposition $W^{\prime} \in \Omega^{\prime}$ and, when $W^{\prime} \in \Omega_{0}$, leave the basis $\left(m e_{1}, m e_{2}\right)$ unchanged. We will always assume $W^{\prime}=\left(W_{0}^{\prime}, \ldots, W_{2 m-2}^{\prime}\right)$, with $W_{k}^{\prime}=W_{k}$ for all blocks $W_{k}$ not involved in the procedure, and with $W_{i}^{\prime}$ and $W_{j}^{\prime}$ defined as above for the two blocks $W_{i}$ and $W_{j}$ involved in the procedure.

Four lemmas. We will often only consider $W \in \Omega_{0}^{n u}$ when $\Omega_{0}^{u}=\emptyset$ (with one exception in Case 3 of Claim C). The reason for this is to ensure that if a swapping procedure applied to $W$ results in a new product decomposition $W^{\prime} \in \Omega_{0}$, then $W^{\prime} \in \Omega_{0}^{n u}$ is guaranteed, and hence the more powerful Lemma 3.3 is available (instead of the weaker Lemma 3.2).

The following lemma will be used in Case 3 of Claim $\mathrm{C}$ to avoid having to consider a $W^{\prime \prime} \in \Omega_{0}^{n u}$ when $\Omega_{0}^{u} \neq \emptyset$.

Lemma 5.1. Let $W \in \Omega_{0}^{u}, U \in \mathcal{C}_{1}$ and $V_{1}, V_{2} \in \mathcal{C}_{0}$ be distinct. Suppose there exist $X \mid U$ and $Y_{1} \mid V_{1}$ such that swapping $X$ for $Y_{1}$ yields a new product decomposition $W^{\prime} \in \Omega^{\prime}$ with the new block $U^{\prime}=X^{-1} U Y_{1}$ in $W^{\prime}$ having 
$\sigma\left(U^{\prime}\right) \neq \sigma(U)$. If $Y_{2} \mid Y_{1}^{-1} V_{1}$ and $Z \mid V_{2}$ are nontrivial subsequences such that swapping $Y_{2}$ for $Z$ in $W$ yields a new product decomposition $W^{\prime \prime} \in \Omega_{0}$, then $W^{\prime \prime} \in \Omega_{0}^{u}$.

Proof. Assume by contradiction that $W^{\prime \prime} \in \Omega_{0}^{n u}$, so that w.l.o.g. $\widetilde{\sigma}\left(W^{\prime \prime}\right)$ $=f_{1}^{m-1} f_{2}^{m-1}\left(f_{1}+f_{2}\right)$ with $\sigma(U)=f_{1}$ (since $\sigma(U)$ is a maximal multiplicity term in $\widetilde{\sigma}(W)$ and all blocks involved in the swap resulting in $W^{\prime \prime}$ are of nonmaximal multiplicity, it follows that $\sigma\left(U^{\prime \prime}\right)=\sigma(U)$ must be a maximal multiplicity term in $\widetilde{\sigma}\left(W^{\prime \prime}\right)$ as well). Since $m \geq 4$ (so that $f_{2} f_{1}^{m-1} \mid \widetilde{\sigma}(W)$ ), let $\sigma\left(V_{1}\right)=C f_{1}+f_{2}$ with $C \in[0, m-1]$. By hypothesis, we may swap $Y_{1} \mid V_{1}^{\prime \prime}=$ $Y_{2}^{-1} V_{1} Z$ for $X \mid U^{\prime \prime}=U$ to obtain a new product decomposition $W^{\prime \prime \prime} \in \Omega^{\prime}$, with new respective terms $V_{1}^{\prime \prime \prime}$ and $U^{\prime \prime \prime}$. Since (by hypothesis) swapping $X$ for $Y_{1}$ in $W$ yields a new product decomposition $W^{\prime} \in \Omega^{\prime}$ such that the new block $U^{\prime}=X^{-1} U Y_{1}$ in $W^{\prime}$ has $\sigma\left(U^{\prime}\right) \neq \sigma(U)$, it follows from Lemma 3.12 that $\sigma\left(U^{\prime \prime \prime}\right)=\sigma\left(U^{\prime}\right)=C f_{1}+f_{2}$ and $\sigma\left(V_{1}^{\prime \prime \prime}\right)=\sigma\left(V_{1}^{\prime \prime}\right)+(1-C) f_{1}-f_{2}$.

Suppose $\sigma\left(V_{1}^{\prime \prime}\right)=f_{2}$. Then, from the above paragraph, we conclude that

$$
\widetilde{\sigma}\left(W^{\prime \prime \prime}\right)=f_{2}^{m-2}\left(f_{1}+f_{2}\right)\left((1-C) f_{1}\right) f_{1}^{m-2}\left(C f_{1}+f_{2}\right) .
$$

Thus, since $\tilde{\sigma}\left(W^{\prime \prime \prime}\right) \in \Upsilon(\operatorname{Ker}(\varphi))$ and $m \geq 4$, it follows that $C=0$, whence $\sigma\left(V_{1}^{\prime \prime}\right)=f_{2}=C f_{1}+f_{2}=\sigma\left(V_{1}\right)$. However, this implies that $\tilde{\sigma}(W)=\tilde{\sigma}\left(W^{\prime \prime}\right) \in \Upsilon_{0}^{n u}$, contrary to $W \in \Omega_{0}^{u}$. So we may assume instead that $\sigma\left(V_{1}^{\prime \prime}\right)=f_{1}+f_{2}$ (note $\sigma\left(V_{1}^{\prime \prime}\right) \neq f_{1}$, since $\sigma(U)=f_{1}, U \in \mathcal{C}_{1}(W)$ and no terms from $\mathcal{C}_{1}(W)$ were involved in the swap resulting in $\left.W^{\prime \prime}\right)$.

In this case, we instead conclude that

$$
\widetilde{\sigma}\left(W^{\prime \prime \prime}\right)=f_{2}^{m-1}\left((2-C) f_{1}\right) f_{1}^{m-2}\left(C f_{1}+f_{2}\right) .
$$

Thus, since $\tilde{\sigma}\left(W^{\prime \prime \prime}\right) \in \Upsilon(\operatorname{Ker}(\varphi))$ and $m \geq 3$, we conclude that $C=1=$ $2-C$, and once more $\sigma\left(V_{1}^{\prime \prime}\right)=\sigma\left(V_{1}\right)$, yielding the same contradiction as in the previous paragraph, completing the proof.

The next two lemmas will often be used in conjunction, and will form one of our main swapping strategy arguments used for Claims A and B. Note that Lemma 5.2(i) gives a strong structural description as well as a term of multiplicity at least $\left(\left|\mathcal{D}_{1}\right|+1\right) n-1$ in $S$, while Lemma 5.2(ii) allows us to invoke Lemma 5.3 .

Lemma 5.2. Let $W \in \Omega_{0}$ and, if $\Omega_{0}^{u} \neq \emptyset$, assume that $W \in \Omega_{0}^{u}$. Let $\mathcal{D}_{1}, \mathcal{D}_{2} \subset \mathcal{A}_{2}^{*}$ be such that, for each (relevant) $i \in[0,2]$, there do not exist $U \in \mathcal{D}_{1}$ and $V \in \mathcal{D}_{2}$ with $U, V \in \mathcal{C}_{i}$. If either

(a) $\left|\mathcal{D}_{1}\right| \geq 1$ and every type III swap between $x \mid W_{0}^{(2)}$ and $y \mid W_{j}$, with $W_{j} \in \mathcal{D}_{1}$ and $\iota(x)=\iota(y)$, results in a new product decomposition $W^{\prime}$ with $\sigma\left(W_{0}^{\prime}\right)=\sigma\left(W_{0}\right)$, or

(b) $\left|\mathcal{D}_{1}\right| \geq 2$ and $\left|\mathcal{D}_{2}\right| \geq 1$,

then one of the following two statements holds: 
(i) There exist $x_{0} \mid W_{0}^{(2)}, g \in I$ and $\alpha \in \operatorname{Ker}(\varphi)$ such that $\iota\left(x_{0}\right) \equiv$ $g+1 \bmod n, \iota(x)=g$ and $\psi(x)=\alpha$, for all $x \mid x_{0}^{-1} W_{0}^{(2)} \prod_{V \in \mathcal{D}_{1}} V$. In particular, $\mathrm{v}_{g e_{1}+e_{2}+\alpha}(S) \geq\left(\left|\mathcal{D}_{1}\right|+1\right) n-1$.

(ii) There exist $W_{j} \in \mathcal{D}_{1}, X \mid W_{0}^{(2)}$ and $Y \mid W_{j}$ such that $|X|=|Y|$ and $\epsilon^{\prime}(X, Y) \notin\{1, n\}$.

Proof. We assume that (ii) fails and show that (i) holds. During the proof we make implicit use of the fact that $n \geq 3$. If $W_{0} \in \mathcal{C}_{0}$, then choose $f_{2}$ such that $\sigma\left(W_{0}\right)=f_{1}+f_{2}$; if $W \in \Omega_{0}^{n u}$, then choose $f_{2}$ such that $\widetilde{\sigma}(W)=f_{1}^{m-1} f_{2}^{m-1}\left(f_{1}+f_{2}\right)$ (note, in the case $W_{0} \in C_{0}$ and $W \in \Omega_{0}^{n u}$, that this choice of $f_{2}$ agrees with the previous choice), and assume $\mathcal{C}_{1}$ consists of those $W_{i}$ with $\sigma\left(W_{i}\right)=f_{1}$; and if $W_{0} \notin \mathcal{C}_{0}$, then w.l.o.g. assume $W_{0} \in \mathcal{C}_{1}$.

Applying Lemma 3.43 to $\iota\left(W_{0}^{(2)}\right)$ and each $\iota(V)$ with $V \in \mathcal{D}_{1}$, with both sequences considered modulo $n$ (since (ii) fails, the hypothesis of Lemma 3.4. 3 holds with $\{0, a\}$ equal to $\{n, 1\}$ modulo $n)$, we conclude, in view of $\sigma\left(\iota\left(W_{0}^{(2)}\right)\right) \equiv 1 \bmod n$ (and hence $\left|\operatorname{supp}\left(\iota\left(W_{0}^{(2)}\right)\right)\right|>1$ ), that there exist $x_{0} \mid W_{0}^{(2)}$ and $g \in I$ such that $\iota\left(x_{0}\right) \equiv g+1 \bmod n$ and $\iota(x)=g$ for all $x \mid x_{0}^{-1} W_{0}^{(2)} \prod_{V \in \mathcal{D}_{1}} V$. If (a) holds, then performing type III swaps between $W_{0}$ and the $V \in \mathcal{D}_{1}$ completes the proof. Therefore assume (a) fails and (b) holds instead.

CASE 1: $W_{0} \in \mathcal{C}_{0}$. Thus, since $\left|\mathcal{D}_{1}\right|,\left|\mathcal{D}_{2}\right| \geq 1$ (and in view of the hypotheses), let $U \in \mathcal{A}_{2}^{*} \cap\left(\mathcal{D}_{1} \cup \mathcal{D}_{2}\right)$ with $\sigma(U)=f_{1}$ and let $V \in \mathcal{A}_{2}^{*} \cap\left(\mathcal{D}_{1} \cup \mathcal{D}_{2}\right)$ with $\sigma(V)=C f_{1}+f_{2}$ for some $C \in \mathbb{Z}$. Performing a type II swap between some fixed $u \mid U$ and each $x \mid x_{0}^{-1} W_{0}^{(2)}$ (using the same fixed subsequence $R \mid W_{0}^{(1)}$ in every swap, which is possible since $\iota(x)=g$ for all $\left.x \mid x_{0}^{-1} W_{0}^{(2)}\right)$, we conclude from either Lemma 3.1.2 (since $\left.\sigma\left(W_{0}\right)=f_{1}+f_{2}\right)$ or Lemma 3.2. 4 that $\psi_{1}$ is constant on $x_{0}^{-1} W_{0}^{(2)}$. Likewise performing a type II swap between some fixed $v \mid V$ and each $x \mid x_{0}^{-1} W_{0}^{(2)}$, we conclude from either Lemma 3.1.3 or Lemma 3.25 that $\psi_{2}$ is constant on $x_{0}^{-1} W_{0}^{(2)}$. Consequently, $\psi(x)=\alpha$ (say) for all $x \mid x_{0}^{-1} W_{0}^{(2)}$.

Suppose $W \in \Omega_{0}^{n u}$. Then $\mathcal{D}_{1} \subset \mathcal{A}_{2}^{*} \cap \mathcal{C}_{i}$ for some $i \in\{1,2\}$ (in view of the hypotheses of Case 1 and the lemma), and performing type III swaps between the $Z \in \mathcal{D}_{1}$, we conclude, in view of $\left|\mathcal{D}_{1}\right| \geq 2$, that $\iota(x)=g$ for all $x \mid x_{0}^{-1} W_{0}^{(2)} \prod_{V \in \mathcal{D}_{1}} V$, and by Lemma 3.3 .1 or 3.32 , that $\psi(x)=\alpha^{\prime}$ (say) for all $x \mid \prod_{V \in \mathcal{D}_{1}} V$. Further, applying type III swaps between $W_{0}$ and any $Z \in \mathcal{D}_{1}$, we conclude from Lemma 3.4 .3 and either Lemma 3.3.4 or 3.3.5 that $\alpha=\alpha^{\prime}$, completing the proof. So we may assume $W \in \Omega_{0}^{u}$.

If $\mathcal{D}_{1} \subset \mathcal{C}_{1}$, then repeating the argument of the previous paragraph using Lemma 3.1 in place of Lemma 3.3 completes the proof. Therefore we may 
assume $\mathcal{D}_{1} \subset \mathcal{C}_{0}$. Let $Z \in \mathcal{D}_{1}$ and $z \mid Z$. We proceed to show $\psi(z)=\alpha$, which, since $z \mid Z \in \mathcal{D}_{1}$ is arbitrary, will complete the proof.

If performing a type III swap between $z \mid Z$ and some $x \mid x_{0}^{-1} W_{0}^{(2)}$ results in a new product decomposition $W^{\prime} \in \Omega_{0}^{u}$, then $W_{0}^{\prime}, Z^{\prime} \in \mathcal{C}_{0}\left(\right.$ as $\left.W_{0}, Z \in \mathcal{C}_{0}\right)$ and, repeating the arguments of the first paragraph of Case 1 for $W^{\prime}$, we conclude that $\psi(z)=\alpha$. If $W^{\prime} \in \Omega_{0}^{n u}$, then we can choose a new $f_{2}$ such that $\tilde{\sigma}\left(W^{\prime}\right)=f_{1}^{m-1} f_{2}^{m-1}\left(f_{1}+f_{2}\right)$. If also $W_{0}^{\prime} \in \mathcal{C}_{0}$, then $\sigma\left(W_{0}^{\prime}\right)=f_{1}+f_{2}$, and repeating the arguments of the first paragraph of Case 1 for $W^{\prime}$ shows $\psi(z)=\alpha$. Therefore suppose $W^{\prime} \in \Omega_{0}^{n u}$ and $\sigma\left(W_{0}^{\prime}\right)=f_{2}$. In view of Lemma 3.1 . 3, we have $\alpha-\psi(z) \in\left\langle f_{1}\right\rangle$. However, if $\alpha \neq \psi(z)$, then performing a type II swap between some $y \mid U^{\prime}=U$ and both $z \mid W_{0}^{\prime}$ and $z^{\prime} \mid W_{0}^{\prime}$, where $\iota\left(z^{\prime}\right)=g$ and $\psi\left(z^{\prime}\right)=\alpha$, we conclude from Lemma 3.2 .3 that

$$
\epsilon n e_{1}+\sigma(\psi(R))-\psi(y)+\{\psi(z), \alpha\}=\left\{0, f_{2}-f_{1}\right\},
$$

where $\epsilon=\epsilon(z, y)=\epsilon\left(z^{\prime}, y\right)$ (in view of $\iota(z)=\iota\left(z^{\prime}\right)=g$ ) and $R$ is the same fixed subsequence of $W_{0}^{\prime(1)}$ used in both swaps (also possible since $\iota(z)=$ $\left.\iota\left(z^{\prime}\right)=g\right)$. Hence $\psi(z)-\alpha= \pm\left(f_{2}-f_{1}\right)$, contradicting that $\alpha-\psi(z) \in\left\langle f_{1}\right\rangle$, and completing Case 1.

CASE 2: $W_{0} \notin \mathcal{C}_{0}$ and $W \in \Omega_{0}^{n u}$. Then $W_{0} \in \mathcal{C}_{1}$ (by our normalizing assumptions). If there is $Z \in \mathcal{D}_{1} \cap \mathcal{C}_{0}$ and $\mathcal{D}_{1} \cap \mathcal{C}_{2}=\emptyset$, then, in view of Lemma 3.3 , we may assume that performing any type III swap between $z \mid Z$ and $x \mid x_{0}^{-1} W_{0}^{(2)}$ results in a product decomposition $W^{\prime}$ with $\sigma\left(W_{0}^{\prime}\right)=\sigma\left(W_{0}\right)$, else Case 1 applied to $W^{\prime}$ completes the proof. Note that Lemma 3.31 guarantees the same for any $Z \in \mathcal{D}_{1} \cap \mathcal{C}_{1}$. Thus if $\mathcal{D}_{1} \cap \mathcal{C}_{2}=\emptyset$, then (a) holds, contrary to assumption, and so we may assume instead that $\mathcal{D}_{1} \cap \mathcal{C}_{2} \neq \emptyset$.

Suppose there is $Z \in \mathcal{D}_{2}$ with $\sigma(Z)=f_{1}+f_{2}$. Then performing type II swaps between some $z \mid Z$ and each $x \mid x_{0}^{-1} W_{0}^{(2)}$ (using the same $R \mid W_{0}^{(1)}$ for every swap, which is possible since $\iota(x)=g$ for all $\left.x \mid x_{0}^{-1} W_{0}^{(2)}\right)$, we conclude from Lemma 3.24 that $\psi_{1}$ is constant on $x_{0}^{-1} W_{0}^{(2)}$. If we perform type III swaps between $U$ and $W_{0}$ with $U \in \mathcal{D}_{1} \cap \mathcal{C}_{2}$, then we conclude from Lemmas 3.23 and 3.4 .3 that there is $u_{0} \mid x_{0}^{-1} W_{0}^{(2)} U$ such that $\psi(x)=\alpha$ (say) for all $x \mid u_{0}^{-1} x_{0}^{-1} W_{0}^{(2)} U$ and $\psi\left(u_{0}\right)=\alpha$ or $\alpha \pm\left(f_{2}-f_{1}\right)$; moreover, $\psi\left(u_{0}\right)=\alpha+f_{2}-f_{1}$ is possible only if $u_{0} \mid U$, and $\psi\left(u_{0}\right)=\alpha-\left(f_{2}-f_{1}\right)$ is possible only if $u_{0} \mid W_{0}^{(2)}$. Thus, as $\psi_{1}$ is constant on $x_{0}^{-1} W_{0}^{(2)}$ and $\psi_{1}(\alpha) \neq$ $\psi_{1}\left(\alpha+f_{2}-f_{1}\right)$, we conclude that $\psi(x)=\alpha$ for all $x \mid x_{0}^{-1} W_{0}^{(2)}$. If $u_{0} \mid U$ with $\psi\left(u_{0}\right)=\alpha+f_{2}-f_{1}$, then swapping $u_{0} \mid U$ for $x \mid x_{0}^{-1} W_{0}^{(2)}$ results in a new product decomposition $W^{\prime}$ such that $\widetilde{\sigma}\left(W^{\prime}\right)=\widetilde{\sigma}(W), \sigma\left(W_{0}^{\prime}\right)=f_{2}$, and $\psi_{2}$ is not constant on $x_{0}^{-1} W_{0}^{\prime(2)}$. However repeating the argument from the beginning of the paragraph for $W^{\prime}$, using Lemma 3.2 .5 in place of Lemma 3.2.4, 
we see that $\psi_{2}$ must be constant on $x_{0}^{-1} W_{0}^{\prime(2)}$, a contradiction. Thus we see that any type III swap between $u \mid U \in \mathcal{D}_{1} \cap \mathcal{C}_{2}$ and $x \mid x_{0}^{-1} W_{0}^{(2)}$ results in a product decomposition $W^{\prime}$ with $\sigma\left(W_{0}^{\prime}\right)=\sigma\left(W_{0}\right)$. As a result, since $Z \in \mathcal{D}_{2}$ with $\sigma(Z)=f_{1}+f_{2}$, it follows from Lemma 3.3.1 that (a) holds, contrary to assumption. So we may assume $\mathcal{D}_{2} \cap \mathcal{C}_{0}$ is empty. Thus, in view of $\mathcal{D}_{1} \cap \mathcal{C}_{2} \neq \emptyset$ and the hypotheses, it follows that there is $U \in \mathcal{D}_{2} \cap \mathcal{C}_{1}$.

Performing type II swaps between some $y \mid U$ and each $x \mid x_{0}^{-1} W_{0}^{(2)}$ (using the same $R \mid W_{0}^{(1)}$ for every swap), we conclude from Lemma 3.2 .1 that $\psi_{1}$ is constant on $x_{0}^{-1} W_{0}^{(2)}$. Consequently, performing type III swaps between $W_{0}$ and each $V_{i} \in \mathcal{D}_{1} \cap \mathcal{C}_{2}$, we conclude from Lemmas 3.2 .3 and 3.4 .3 that there exists $v_{i} \mid V_{i}$ such that $\psi(x)=\alpha$ (say) for all $x \mid v_{i}^{-1} x_{0}^{-1} W_{0}^{(2)} V_{i}$; moreover, $\psi\left(v_{i}\right)=\alpha$ or $\alpha+f_{2}-f_{1}$. If there is $Z \in \mathcal{D}_{1} \cap \mathcal{C}_{0}$, then, performing type III swaps between the $x \mid x_{0}^{-1} W_{0}^{(2)}$ and $z \mid Z$, and between the $x \mid V_{i} \in \mathcal{D}_{1} \cap \mathcal{C}_{2}$ and $z \mid Z$, we conclude from Lemmas 3.3.4 and 3.3.5 that $\psi(x)=\alpha$ for all $x \mid Z$.

If $Z \in \mathcal{D}_{1} \cap \mathcal{C}_{0}$ does not exist, then $\left|\mathcal{D}_{1}\right| \geq 2$ and $\left|\mathcal{D}_{2} \cap \mathcal{C}_{1}\right| \geq 1$ ensure $\left|\mathcal{D}_{1} \cap \mathcal{C}_{2}\right| \geq 2$, and, performing type III swaps between the $V \in \mathcal{D}_{1} \cap \mathcal{C}_{2}$, we conclude from Lemma 3.32 that $\psi(x)=\alpha$ for all $x \mid V$ with $V \in \mathcal{D}_{1} \cap \mathcal{C}_{2}$, completing the proof. On the other hand, if there is $Z \in \mathcal{D}_{1} \cap \mathcal{C}_{0}$, then applying type III swaps between $Z$ and each $V_{i} \in \mathcal{D}_{1} \cap \mathcal{C}_{2}$, we conclude from Lemma 3.25 that $\psi_{2}$ is constant on $V_{i}$ and $Z$; consequently, since $\psi\left(v_{i}\right)=\alpha$ or $\alpha+f_{2}-f_{1}$, and since $\psi(v)=\alpha$ for all $v \mid v_{i}^{-1} V_{i}$, we conclude that $\psi\left(v_{i}\right)=\alpha$ as well, completing the proof.

CASE 3: $W_{0} \notin \mathcal{C}_{0}$ and $W \in \Omega_{0}^{u}$. Then $W_{0} \in \mathcal{C}_{1}$ and $\mathcal{D}_{1} \subset \mathcal{C}_{0}$ (else (a) holds in view of Lemma 3.1.1). Hence, since $\left|\mathcal{D}_{2}\right| \geq 1$, there is $U \in \mathcal{A}_{2}^{*} \cap \mathcal{C}_{1}$. Performing type II swaps between each $x \mid x_{0}^{-1} W_{0}$ and some fixed $u \mid U$ (using the same fixed sequence $R \mid W_{0}^{(1)}$ in each swap), it follows from Lemma 3.1. 1 that $\psi(x)=\alpha$ (say) for all $x \mid x_{0}^{-1} W_{0}^{(2)}$. Let $V_{i} \in \mathcal{D}_{1}$. Performing type III swaps between $W_{0}$ and $V_{i}$, we conclude from Lemmas 3.1.2 and 3.4. 3 that $\psi(z)=\alpha$ for all $z \mid v_{i}^{-1} V_{i}$, for some $v_{i} \mid V_{i}$; moreover, either $\psi\left(v_{i}\right)=\alpha$ or $\psi\left(v_{i}\right)=\alpha-\sigma\left(W_{0}\right)+\sigma\left(V_{i}\right)$. However, in the latter case, since $V_{i} \in \mathcal{C}_{0}$ and $W_{0} \in \mathcal{C}_{1}$ (so that $\sigma\left(W_{0}\right)=f_{1}$ and $\sigma\left(V_{i}\right)=C f_{1}+f_{2}$, for some $C \in \mathbb{Z}$ ), we see that $\psi_{2}\left(v_{i}\right) \neq \psi_{2}(\alpha)$. Since $\left|\mathcal{D}_{1}\right| \geq 2$, performing type III swaps between the $V_{i} \in \mathcal{D}_{1}$, we conclude from Lemma 3.1.3 that $\psi_{2}$ is constant on each $V_{i}$, whence $\psi_{2}\left(v_{i}\right) \neq \psi_{2}(\alpha)$ is impossible. Thus $\psi(z)=\alpha$ for all $z \mid V_{i}$ with $V_{i} \in \mathcal{D}_{1}$, completing the proof.

Lemma 5.3 allows us to deduce detailed information concerning the values of $\psi$ on $W_{0}^{(1)}$. Depending on $\sigma\left(W_{j}\right)$ and $\sigma\left(W_{0}\right)$, the appropriate part of 
Lemma 3.1 or 3.2 will ensure that one of the hypotheses in item 1,2 , or 3 below holds.

Lemma 5.3. Let $W \in \Omega_{0}$ and $W_{j} \in \mathcal{A}_{2}^{*}$ be such that there are $Y \mid W_{j}$ and $X \mid W_{0}^{(2)}$ with $|X|=|Y|$ and $\epsilon^{\prime}(X, Y) \notin\{1, n\}$, and set

$\mathcal{D}=\left\{W^{\prime} \in \Omega^{\prime} \mid W^{\prime}\right.$ is the result of performing a type II swap between $X \mid W_{0}$ and $\left.Y \mid W_{j}\right\}$.

1. If $\sigma\left(W_{j}^{\prime}\right)-\sigma\left(W_{j}\right)=0$ for all $W^{\prime} \in \mathcal{D}$, then $\left|\operatorname{supp}\left(\psi\left(W_{0}^{(1)}\right)\right)\right|=1$.

2. If $\sigma\left(W_{j}^{\prime}\right)-\sigma\left(W_{j}\right) \in\left\langle f_{i}\right\rangle$, where $i \in\{1,2\}$, for all $W^{\prime} \in \mathcal{D}$, then $\left|\operatorname{supp}\left(\psi_{3-i}\left(W_{0}^{(1)}\right)\right)\right|=1$.

3. If $\sigma\left(W_{j}^{\prime}\right)-\sigma\left(W_{j}\right) \in\{0, F\}$ for all $W^{\prime} \in \mathcal{D}$, where $F \in \operatorname{Ker}(\varphi)$, then $\operatorname{supp}\left(\psi\left(W_{0}^{(1)}\right)\right)=\{\gamma, \beta\}$ for some $\gamma, \beta \in \operatorname{Ker}(\varphi)$ with $\gamma-\beta \in\{0, \pm F\}$.

Proof. 1. By hypothesis, there is only one possibility for $\sigma(\psi(R))$, where $R \mid W_{0}^{(1)}$ is any subsequence with $|R|=n-\epsilon^{\prime}(X, Y)$. Furthermore, we have $1 \leq|R| \leq n-2<\left|\psi\left(W_{0}^{(1)}\right)\right|$, and thus item 1 follows from Lemma 3.5.3 applied to $\psi\left(W_{0}^{(1)}\right)$.

2. The argument is analogous to that for item 1, using the group $\operatorname{Ker}(\varphi) /\left\langle f_{i}\right\rangle \cong\left\langle f_{3-i}\right\rangle$ in place of $\operatorname{Ker}(\varphi)$.

3. By the arguments for item 1, replacing Lemma 3.5.3 by Lemma 3.5.1, we conclude that $\psi\left(W_{0}^{(1)}\right)=\gamma^{l} \beta^{n-1-l}$ (say), where $l \geq n-1-l \geq 1$ and $\gamma \neq \beta$ (else the assertion holds); moreover,

$$
\begin{aligned}
\epsilon(X, Y) n e_{1}+\sigma(\psi(X))- & \sigma(\psi(Y))+\min \{t, l\} \cdot \gamma \\
& +(t-\min \{t, l\}) \cdot \beta+\{0, \beta-\gamma\}=\{0, F\},
\end{aligned}
$$

where $t=n-\epsilon^{\prime}(X, Y)$. Thus $\beta-\gamma= \pm F$, as desired.

The following lemma encapsulates an alignment argument for the $\iota$ values that forces them to live in near disjoint intervals. It will be a key part of the more difficult portions of Claim C.

LemmA 5.4. Let $W \in \Omega_{0}$, let $\mathcal{D} \subset \mathcal{A}_{2}^{*}$ be nonempty, and let $Z \mid W_{0}^{(2)}$ be nontrivial. For $x \mid S$, let $\psi_{0}(x)=\psi(x)$, and for $x \in \operatorname{Ker}(\varphi)$, let $\psi_{0}$ be the identity map. Let $i \in\{0,1,2\}$. If $\psi_{i}\left(n e_{1}\right) \neq 0$ and

$$
\psi_{i}(x)-\psi_{i}(y)+\psi_{i}\left(\epsilon(x, y) n e_{1}\right)=0
$$

for every $x \mid Z$ and $y \mid U \in \mathcal{D}$, then there exist intervals $J_{1}, J_{2}$ and $J_{3}$ of $\mathbb{Z}$ with either

$$
\begin{aligned}
\operatorname{supp}\left(\iota\left(\prod_{U \in \mathcal{D}} U\right)\right) \subset J_{3}, \quad \operatorname{supp}(\iota(Z)) \subset J_{1} \cup J_{2}, \quad \text { and } \\
\max J_{1} \leq \min J_{3} \leq \max J_{3}<\min J_{2}, \quad \text { or }
\end{aligned}
$$




$$
\begin{aligned}
\operatorname{supp}(\iota(Z)) \subset J_{3}, \quad \operatorname{supp}\left(\iota\left(\prod_{U \in \mathcal{D}} U\right)\right) \subset J_{1} \cup J_{2}, \quad \text { and } \\
\quad \max J_{1}<\min J_{3} \leq \max J_{3} \leq \min J_{2} .
\end{aligned}
$$

Moreover, I can be chosen such that:

1. $\min I$ is congruent to an element in $\iota(Z)$ modulo $n$,

2. $\iota(x) \leq \iota(y)$ and $\epsilon(x, y)=0$ for all $x \mid Z$ and $y \mid U \in \mathcal{D}$,

3. $\psi_{i}(x)=\psi_{i}(y)$ for all $x y \mid Z \prod_{U \in \mathcal{D}} U$.

Proof. Observe, for $x y \mid S_{2}$, that

$$
\epsilon(x, y)= \begin{cases}0, & \iota(x) \leq \iota(y) \\ 1, & \iota(x)>\iota(y)\end{cases}
$$

Consequently, we conclude from 10 that

$$
\psi_{i}(x)=\psi_{i}(y)
$$

for all $x \mid Z$ and $y \mid U \in \mathcal{D}$ with $\iota(x) \leq \iota(y)$, and that

$$
\psi_{i}(x)=\psi_{i}(y)-\psi_{i}\left(n e_{1}\right)
$$

for all $x \mid Z$ and $y \mid U \in \mathcal{D}$ with $\iota(x)>\iota(y)$.

If there do not exist $x \mid Z$ and $y y^{\prime} \mid \prod_{U \in \mathcal{D}} U$ with $\iota(x) \leq \iota(y)$ and $\iota(x)>$ $\iota\left(y^{\prime}\right)$, then, for every $x \mid Z$, we have either $\iota(x) \leq \iota(y)$ for all $y \mid \prod_{U \in \mathcal{D}} U$, or $\iota(x)>\iota(y)$ for all $y \mid \prod_{U \in \mathcal{D}} U$. Thus we see that (11) holds (with $J_{3}=$ $\left[\min \left(\operatorname{supp}\left(\iota\left(\prod_{U \in \mathcal{D}} U\right)\right)\right), \max \left(\operatorname{supp}\left(\iota\left(\prod_{U \in \mathcal{D}} U\right)\right)\right)\right], J_{1}$ being any nonempty interval containing those $\iota(x)$ with $\iota(x) \leq \iota(y)$ for all $y \mid \prod_{U \in \mathcal{D}} U$ and $\max J_{1} \leq \min J_{3}$, and $J_{2}$ being any nonempty interval containing those $\iota(x)$ with $\iota(x)>\iota(y)$ for all $y \mid \prod_{U \in \mathcal{D}} U$ and $\left.\min J_{2}>\max J_{3}\right)$.

Now instead let $x \mid Z$ and $y y^{\prime} \mid \prod_{U \in \mathcal{D}} U$ with $\iota(x) \leq \iota(y)$ and $\iota(x)>\iota\left(y^{\prime}\right)$, and factor $\prod_{U \in \mathcal{D}} U=J_{1}^{\prime} J_{2}^{\prime}$, where $J_{1}^{\prime}$ are those terms $a \mid \prod_{U \in \mathcal{D}} U$ with $\iota(a)<\iota(x)$ and $J_{2}^{\prime}$ are those terms $b \mid \prod_{U \in \mathcal{D}} U$ with $\iota(b) \geq \iota(x)$. By assumption, both $J_{i}^{\prime}$ are nontrivial. Moreover, from (14) and (15) and $\psi_{i}\left(n e_{1}\right) \neq 0$, we see that

$$
\psi_{i}(b)=\psi_{i}(x)
$$

and

$$
\psi_{i}(a)=\psi_{i}(x)+\psi_{i}\left(n e_{1}\right) \neq \psi_{i}(x),
$$

for all $a \mid J_{1}^{\prime}$ and $b \mid J_{2}^{\prime}$. Thus $\psi_{i}$ is constant on $J_{1}^{\prime}$ and also on $J_{2}^{\prime}$ but the two values assumed are distinct. If there were $x^{\prime} \mid Z$ such that $\iota\left(x^{\prime}\right) \leq$ $\max \left(\operatorname{supp}\left(\iota\left(J_{1}^{\prime}\right)\right)\right)$, then by (14) and (16) we would conclude that $\psi_{i}\left(x^{\prime}\right)=$ $\psi_{i}(b)=\psi_{i}(x)$, where $b$ is any term of $J_{2}^{\prime}$, while from (17) and also (14), applied between $x^{\prime}$ and $\max \left(\operatorname{supp}\left(\iota\left(J_{1}^{\prime}\right)\right)\right):=a_{0}$, we would conclude that $\psi_{i}\left(x^{\prime}\right)=\psi_{i}\left(a_{0}\right)=\psi_{i}(x)+\psi_{i}\left(n e_{1}\right) \neq \psi_{i}(x)$, a contradiction to what we have 
just seen. We likewise obtain a contradiction if there were $x^{\prime} \mid Z$ such that $\iota\left(x^{\prime}\right)>\min \left(\operatorname{supp}\left(\iota\left(J_{2}^{\prime}\right)\right)\right)$. Therefore we see that 12$)$ holds with

$$
\begin{aligned}
& J_{1}=\left[\min \left(\operatorname{supp}\left(\iota\left(J_{1}^{\prime}\right)\right)\right), \max \left(\operatorname{supp}\left(\iota\left(J_{1}^{\prime}\right)\right)\right)\right], \\
& J_{2}=\left[\min \left(\operatorname{supp}\left(\iota\left(J_{2}^{\prime}\right)\right)\right), \max \left(\operatorname{supp}\left(\iota\left(J_{2}^{\prime}\right)\right)\right)\right], \\
& J_{3}=[\min (\operatorname{supp}(\iota(Z))), \max (\operatorname{supp}(\iota(Z)))] .
\end{aligned}
$$

Choosing $I$ such that $\min I$ is congruent to $\min (\operatorname{supp}(\iota(Z)))$ modulo $n$, if either (12) holds or else (11) holds with $\operatorname{supp}(\iota(Z)) \cap J_{2}=\emptyset$, and congruent to $\min \left(\operatorname{supp}(\iota(Z)) \cap J_{2}\right)$ otherwise, the remaining properties follow in view of (13) and (14).

Now we choose a product decomposition $W \in \Omega_{0}$, and if $\Omega_{0}^{u} \neq \emptyset$, we assume that $W \in \Omega_{0}^{u}$.

Claim A. h $\left(S_{1}\right) \geq\left|S_{1}\right|-1$.

Proof. We need to show that there exists $x_{0} \mid S_{1}$ such that $\psi(x)=\psi(y)$ for all $x y \mid x_{0}^{-1} S_{1}$. We divide the proof into four main cases. In many of the cases, we do partial work towards showing $\mathrm{h}\left(S_{1}\right)=\left|S_{1}\right|$, which will later be utilized in Claim B.

CASE 1: $\left|\mathcal{A}_{1}\right|=1$. In this case, we will show that $\mathrm{h}\left(S_{1}\right)=\left|S_{1}\right|$.

Suppose $W_{0} \in \mathcal{C}_{0}$. Then we may choose $f_{2}$ such that $\sigma\left(W_{0}\right)=f_{1}+f_{2}$, and if $\Omega_{0}^{u}=\emptyset$, such that $\widetilde{\sigma}(W)=f_{1}^{m-1} f_{2}^{m-1}\left(f_{1}+f_{2}\right)$ also. Let $\mathcal{D}_{1}$ be those blocks $W_{i}$ with $\sigma\left(W_{i}\right)=f_{1}$ and let $\mathcal{D}_{2}$ be all other blocks from $\mathcal{A}_{2}^{*}$. Note $\left|\mathcal{D}_{1}\right|=\left|\mathcal{D}_{2}\right|=m-1$ in view of $\left|\mathcal{A}_{1}\right|=1$. Applying Lemma 5.2, we see that Lemma 5.2(ii) must hold, else $g e_{1}+e_{2}+\alpha$ will have multiplicity at least $m n-1$ in $S$, as desired. Performing type II swaps between the $X \mid W_{0}^{(2)}$ and $Y \mid W_{j}$ given by Lemma 5.2(ii), we conclude, from Lemmas 5.3.2 and either 3.12 ( since $\left.\sigma\left(W_{0}\right)=f_{1}+f_{2}\right)$ or 3.2 , that $\psi_{1}$ is constant on $W_{0}^{(1)}$. However, reversing the roles of $\mathcal{D}_{1}$ and $\mathcal{D}_{2}$ and repeating the above argument using Lemmas 3.1.3 and 3.2.5 in place of Lemmas 3.1.2 and 3.2.4, we conclude that $\psi_{2}$ is also constant on $W_{0}^{(1)}$, whence $\psi$ is constant on $W_{0}^{(1)}$, completing the proof of Case 1 . So we may assume $W_{0} \notin \mathcal{C}_{0}$.

Suppose $\Omega_{0}^{u}=\emptyset$. Then we may assume that $\widetilde{\sigma}(W)=f_{1}^{m-1} f_{2}^{m-1}\left(f_{1}+f_{2}\right)$, $\mathcal{C}_{1}$ consists of those blocks $W_{i}$ with $\sigma\left(W_{i}\right)=f_{1}$, and $\sigma\left(W_{0}\right)=f_{1}$. Let $\mathcal{D}_{1}=\mathcal{C}_{2}$ and $\mathcal{D}_{2}=\mathcal{C}_{1}^{*} \cup \mathcal{C}_{0}$. Applying Lemma 5.2, we see that Lemma 5.2(ii) must hold, else there will be a term with multiplicity at least $m n-1$ in $S$, as desired. Thus Lemmas 5.3.3 and 3.2.3 imply that $\operatorname{supp}\left(\psi\left(W_{0}^{(1)}\right)\right)=\{\gamma, \beta\}$ (say) with $\beta-\gamma= \pm\left(f_{2}-f_{1}\right)$ (else Case 1 is complete).

Reversing the roles of $\mathcal{D}_{1}$ and $\mathcal{D}_{2}$ and again applying Lemma 5.2 , we once more see that Lemma 5.2(ii) must hold, else there is a term with multiplicity $m n-1$ in $S$, as desired. Thus Lemma 5.32 and either Lemma 3.2 , 1 or 3.2 .4 
imply that $\psi_{1}$ is constant on $W_{0}^{(1)}$, contradicting that $\beta-\gamma= \pm\left(f_{2}-f_{1}\right)$. So we may assume $\Omega_{0}^{u} \neq \emptyset$.

Let w.l.o.g. $W_{1}, \ldots, W_{m-2}$ be the blocks of $\mathcal{C}_{1}^{*} \cap \mathcal{A}_{2}$, and let $\mathcal{D}_{1}=\mathcal{C}_{1}^{*}$ and $\mathcal{D}_{2}=\mathcal{C}_{0}$. Apply Lemma 5.2. If Lemma 5.2(ii) holds, then Lemmas 5.3.1 and 3.1.1 imply that $\psi$ is constant on $W_{0}^{(1)}$, whence Case 1 is complete. Therefore we may instead assume $\iota(x)=g$ and $\psi(x)=\alpha$ (say) for all terms $x \mid x_{0}^{-1} W_{0}^{(2)} W_{1} \ldots W_{m-2}$, for some $x_{0} \mid W_{0}^{(2)}$ with $\iota\left(x_{0}\right) \equiv g+1 \bmod n$.

Consider $W_{j}$ with $j \geq m-1$. If $\iota\left(W_{j}\right) \neq g^{n}$, then there exist $x \mid W_{0}^{(2)}$ and $y \mid W_{j}$ with $\epsilon^{\prime}(x, y) \notin\{1, n\}$, whence Lemmas 5.3. 3 and 3.1.2 imply that $\operatorname{supp}\left(\psi\left(W_{0}^{(1)}\right)\right)=\{\gamma, \beta\}$ (say) with $\beta-\gamma= \pm F_{j}$ (else Case 1 is complete), where $F_{j}=\left(1-C_{j}\right) f_{1}-f_{2}$ and $\sigma\left(W_{j}\right)=C_{j} f_{1}+f_{2}$.

If $W_{k}$ is another block with $k \geq m-1$ and $\iota\left(W_{k}\right) \neq g^{n}$, then the above paragraph implies that $\beta-\gamma= \pm F_{k}$, where $F_{k}=\left(1-C_{k}\right) f_{1}-f_{2}$ and $\sigma\left(W_{k}\right)=C_{k} f_{1}+f_{2}$. Thus, since $m \geq 3$ and $\beta-\gamma= \pm F_{j}$, we conclude that $F_{j}=F_{k}$ and $C_{j} \equiv C_{k} \bmod m$. As a result, we see that any two blocks $W_{j}$ and $W_{k}$, with $j, k \geq m-1$ and $\iota\left(W_{k}\right), \iota\left(W_{j}\right) \neq g^{n}$, must have $\sigma\left(W_{j}\right)=\sigma\left(W_{k}\right)$. Hence, since $W \in \Omega_{0}^{u}$, we conclude that there are at least two distinct blocks $W_{s}$ and $W_{r}$ with $s, r \geq m-1$ and $\iota\left(W_{s}\right)=\iota\left(W_{r}\right)=g^{n}$. Performing type III swaps between $W_{0}$ and both $W_{s}$ and $W_{r}$, we conclude from Lemmas 3.1.2 and 3.43 that $\psi(x)=\alpha$ for all but at most two terms of $W_{s} W_{r}$, whence $g e_{1}+e_{2}+\alpha$ has multiplicity at least $(m-1) n-1+2 n-2 \geq m n$ in $S$, contradicting that $S \in \mathcal{A}(G)$ and completing Case 1 .

CASE 2: $\left|\mathcal{A}_{1}\right| \geq 2$ and $\Omega_{0}^{u}=\emptyset$. We may w.l.o.g. assume that $\tilde{\sigma}(W)=$ $f_{1}^{m-1} f_{2}^{m-1}\left(f_{1}+f_{2}\right)$, by an appropriate choice of $f_{2}$, whence Claim A follows easily by performing type I swaps between the blocks of $\mathcal{A}_{1}$ and using Lemmas 3.3 and 3.4 . This completes Case 2.

CASE 3: $\left|\mathcal{A}_{1}\right| \geq 2, \Omega_{0}^{u} \neq \emptyset$, and $\left|\mathcal{C}_{1} \cap \mathcal{A}_{1}\right| \geq 1$. In this case, we will moreover show that $\mathrm{h}\left(S_{1}\right)=\left|S_{1}\right|$ unless $\left|\mathcal{A}_{1} \cap \mathcal{C}_{0}\right|=1$ or $\left|\mathcal{A}_{1} \cap \mathcal{C}_{1}\right|=1$, and that $|\operatorname{supp}(\psi(U))|>1$ for $U \in \mathcal{A}_{1} \cap \mathcal{C}_{i}$, where $i \in\{1,2\}$, is only possible when $\left|\mathcal{A}_{1} \cap \mathcal{C}_{i}\right|=1$.

If $U, V \in \mathcal{A}_{1}$ are distinct, then we can perform a type I swap between $U$ and $V$, and by (7) and Lemma 3.1. we conclude that

$$
\begin{array}{lc}
\sigma(\psi(X))-\sigma(\psi(Y))=0 & \text { if } U, V \in \mathcal{C}_{1}, \\
\sigma(\psi(X))-\sigma(\psi(Y)) \in\left\{0,(1-C) f_{1}-f_{2}\right\} & \text { if } U \in \mathcal{C}_{1}, V \in \mathcal{C}_{0} \text { and } \\
& \sigma(V)=C f_{1}+f_{2}, \\
\sigma(\psi(X))-\sigma(\psi(Y)) \in\left\langle f_{1}\right\rangle & \text { if } U, V \in \mathcal{C}_{0},
\end{array}
$$

for $X \mid U$ and $Y \mid V$ with $|X|=|Y|$. 
A1: If $\left|\mathcal{A}_{1} \cap \mathcal{C}_{0}\right| \geq 2$, then using (18) (for all $X$ and $Y$ with $|X|=|Y|=1$ ), we conclude that $\psi(x)-\psi(y) \in\left\langle f_{1}\right\rangle$ for all $x$ and $y$ dividing a block from $\mathcal{A}_{1} \cap \mathcal{C}_{0}$.

A2: If $\left|\mathcal{A}_{1} \cap \mathcal{C}_{1}\right| \geq 2$, then using (18) (for all $X$ and $Y$ with $|X|=|Y|=1$ ) and Lemma 3.4 , we conclude that $\psi(x)=\psi(y)$ for all $x$ and $y$ dividing a block from $\mathcal{A}_{1} \cap \mathcal{C}_{1}$.

In view of $\mathbf{A 2}$, we may assume $\left|\mathcal{A}_{1} \cap \mathcal{C}_{0}\right| \geq 1$, else the proof of Case 3 is complete.

Let $U \in \mathcal{A}_{1} \cap \mathcal{C}_{1}$ and $V \in \mathcal{A}_{1} \cap \mathcal{C}_{0}$ with $U$ and $V$ distinct. Then, using (18) (for all $X$ and $Y$ with $|X|=|Y| \leq 2 \leq n-1$ ) and Lemma 3.4.3, we conclude that $\psi(x)=\alpha$ (say) for all $x \mid x_{0}^{-1} U V$, for some $x_{0} \mid U V$; moreover, $\psi\left(x_{0}\right)=\alpha$ or $\alpha \pm\left((1-C) f_{1}-f_{2}\right)$.

Suppose $x_{0} \mid U$ and $\psi\left(x_{0}\right) \neq \alpha$. Then, in view of $\mathbf{A 2}$, we see that $\left|\mathcal{A}_{1} \cap \mathcal{C}_{1}\right|=1$. Thus performing type I swaps between $U$ and all possible $V \in \mathcal{A}_{1} \cap \mathcal{C}_{0}$ completes Case 3 , for $n \geq 5$ or $U \neq W_{0}$, and, when $n=3$ and $U=W_{0}$, we instead conclude that either $\psi(V)=\alpha^{n}$ or $\psi(V)=\beta^{n}$, where $\psi\left(W_{0}^{(1)}\right)=\alpha \beta$, for all $V \in \mathcal{A}_{1} \cap \mathcal{C}_{0}$. However, if there are $V, V^{\prime} \in \mathcal{A}_{1} \cap \mathcal{C}_{0}$ with $\psi(V)=\alpha^{n}$ and $\psi\left(V^{\prime}\right)=\beta^{n}$ and $\alpha \neq \beta$, then (18) implies that $\beta-\alpha=(1-C) f_{1}-f_{2}$ and $\alpha-\beta=\left(1-C^{\prime}\right) f_{1}-f_{2}$, where $\sigma(V)=C f_{1}+f_{2}$ and $\sigma\left(V^{\prime}\right)=C^{\prime} f_{1}+f_{2}$, from which we conclude that $\left(2-C^{\prime}-C\right) f_{1}-2 f_{2}=0$, contradicting $m \geq 3$. So we may instead assume $x_{0} \mid V$.

In this case, in combination with the results of the previous paragraphs, we find that there is at most one $v_{i} \mid V_{i}$, for each $V_{i} \in \mathcal{A}_{1} \cap \mathcal{C}_{0}$, such that $\psi(x)=\alpha$ for all $x \mid S_{1}$ apart from these $v_{i}$. In this scenario, Case 3 is done unless we have two distinct $V_{1}, V_{2} \in \mathcal{A}_{1} \cap \mathcal{C}_{0}$ such that $\psi\left(v_{1}\right) \neq \alpha$ and $\psi(x)=\alpha$ for all $x \mid v_{1}^{-1} v_{2}^{-1} U V_{1} V_{2}$. However, applying a type I swap between $y \mid U$ and $v_{1} \mid V_{1}$, we conclude from (18) that $\alpha-\psi\left(v_{1}\right)=(1-C) f_{1}-f_{2} \notin\left\langle f_{1}\right\rangle$ for some $C \in \mathbb{Z}$, which, in view of $\alpha \psi\left(v_{1}\right) \mid \psi\left(V_{1}\right)$, contradicts A1. This completes Case 3.

CASE 4: $\left|\mathcal{A}_{1}\right| \geq 2, \Omega_{0}^{u} \neq \emptyset$, and $\left|\mathcal{C}_{1} \cap \mathcal{A}_{1}\right|=0$. In this case, we will moreover show that $\mathrm{h}\left(S_{1}\right)=\left|S_{1}\right|$.

We may w.l.o.g. assume $W_{1}, \ldots, W_{m-1}$ are the blocks in $\mathcal{C}_{1} \cap \mathcal{A}_{2}$. Let $\mathcal{D}_{1}=$ $\mathcal{C}_{1}$ and $\mathcal{D}_{2}=\mathcal{C}_{0}^{*} \cap \mathcal{A}_{2}$. If $\left|\mathcal{D}_{2}\right| \geq 1$, then we can apply Lemma 5.2. Otherwise, in view of Lemma 3.1.2, we may assume hypothesis (a) holds in Lemma 5.2 , else applying Case 3 to the resulting product decomposition $W^{\prime}$ (attained by performing a type III swap that shows (a) fails) would imply, in view of $\left|\mathcal{D}_{2}\right|=0$, that $\psi(x)=\alpha$ (say) for all $x \mid W_{i}^{\prime}=W_{i}$ with $i \in[m, 2 m-2]$, in which case $\sigma\left(W_{i}^{\prime}\right)=n e_{1}+n \alpha$ has multiplicity $m-1$ in $\widetilde{\sigma}\left(W^{\prime}\right)$, contradicting $\tilde{\sigma}\left(W^{\prime}\right)=\tilde{\sigma}(W)$ (in view of Lemma 3.1.2) with $W \in \Omega_{0}^{u}$. Thus, in either case, Lemma 5.2 is available. If Lemma 5.2(i) holds, then $g e_{1}+e_{2}+\alpha$ is a term with multiplicity at least $m n-1$ in $S$ (recall $\left|\mathcal{D}_{1}\right|=\left|\mathcal{C}_{1}\right|=m-1$ ), 
as desired. Therefore there are $X \mid W_{0}^{(2)}$ and $Y \mid W_{j}$, for some $j \in[1, m-1]$, such that $|X|=|Y|$ and $\epsilon^{\prime}(X, Y) \notin\{1, n\}$. Hence Lemmas 5.3.3 and 3.1.2 imply that $\operatorname{supp}\left(\psi\left(W_{0}^{(1)}\right)\right)=\{\gamma, \beta\}$ (say) with $\gamma-\beta \in\{0, \pm F\}$, where $F=(C-1) f_{1}+f_{2}$ and $\sigma\left(W_{0}\right)=C f_{1}+f_{2}$. Since $\left|\mathcal{A}_{1}\right| \geq 2$, let $V \in \mathcal{C}_{0}^{*} \cap \mathcal{A}_{1}$. Performing type I swaps between $W_{0}$ and $V$, we conclude from Lemma 3.1.3 that $\psi_{2}$ is constant on $V W_{0}^{(1)}$, whence $\gamma-\beta \in\{0, \pm F\}$ implies $\gamma=\beta$.

Performing type I swaps among the $V \in \mathcal{C}_{0} \cap \mathcal{A}_{1}$, we conclude from Lemma 3.1.3 that $\psi_{2}(x)=\psi_{2}(\gamma)$ for all $x \mid V \in \mathcal{C}_{0} \cap \mathcal{A}_{1}$. Let $W^{\prime}$ be the product decomposition resulting from performing a type II swap between $X \mid W_{0}$ and $Y \mid W_{j}$ (with $X$ and $Y$ as given by Lemma 5.2(ii) in the previous paragraph). Since $\epsilon^{\prime}(X, Y) \notin\{1, n\}$, we conclude that both blocks $W_{0}^{\prime}$ and $W_{j}^{\prime}$ contain $e_{1}+\gamma$, and thus there is a block $W_{k}^{\prime} \in \mathcal{C}_{1}$ with $k \in\{0, j\}$ and $\left(e_{1}+\gamma\right) \mid W_{k}^{\prime}$. Since $\widetilde{\sigma}\left(W^{\prime}\right)=\widetilde{\sigma}(W)$ (in view of Lemma 3.1 2), performing type I swaps between $W_{k}^{\prime}$ and each distinct block $V^{\prime}=V \in \mathcal{C}_{0}^{*} \cap \mathcal{A}_{1}$, we conclude from Lemma 3.12 that either $\psi(x)=\gamma$ or $\psi(x)=\gamma+\sigma\left(V^{\prime}\right)-\sigma\left(W_{k}^{\prime}\right)$, for each $x \mid V^{\prime}$. However, since $W_{k}^{\prime} \in \mathcal{C}_{1}$ and $V^{\prime} \in \mathcal{C}_{0}$, it follows that the latter contradicts $\psi_{2}$ being constant on $V^{\prime}=V \in \mathcal{C}_{0} \cap \mathcal{A}_{1}$ with value $\psi_{2}(\gamma)$. Therefore we conclude that $\psi(x)=\gamma$ for all $x \mid V^{\prime}$, with $V^{\prime}=V \in \mathcal{C}_{0}^{*} \cap \mathcal{A}_{1}$, whence $\psi(x)=\gamma$ for all $x \mid S_{1}$, as desired, completing Case 4 .

In view of Claim $\mathrm{A}$, we may assume $S_{1}=e_{1}^{\left|S_{1}\right|-1}\left(e_{1}+a\right)$, for some $a \in \operatorname{Ker}(\varphi)$. Let $y_{0}=e_{1}+a$.

Claim B. $\mathrm{h}\left(S_{1}\right)=\left|S_{1}\right|$.

Proof. We assume by contradiction $a \neq 0$. In view of the partial conclusions of Claim A, we may assume $\left|\mathcal{A}_{1}\right| \geq 2$ (in view of Case 1 of Claim A), and, if $\Omega_{0}^{u} \neq \emptyset$, that $\left|\mathcal{A}_{1} \cap \mathcal{C}_{1}\right| \geq 1$ (in view of Case 4 of Claim A). We proceed in four cases.

CASE 1: $\Omega_{0}^{u} \neq \emptyset$ and $y_{0} \mid U$ for some $U \in \mathcal{A}_{1} \cap \mathcal{C}_{1}$. In view of Case 3 of Claim A, we have $\left|\mathcal{A}_{1} \cap \mathcal{C}_{1}\right|=1$. Hence, if $U \neq W_{0}$, then $W_{0} \in \mathcal{C}_{0}$, and performing a type I swap between $y_{0} \mid U$ and some $y \mid W_{0}$ results (in view of Lemma 3.1.2) in a new product decomposition $W^{\prime}$ with $\widetilde{\sigma}\left(W^{\prime}\right)=\widetilde{\sigma}(W)$, $U^{\prime} \in \mathcal{C}_{0}, W_{0}^{\prime} \in \mathcal{C}_{1}, y_{0} \mid W_{0}^{\prime}$ and $W^{\prime}$ also satisfying the hypothesis of Case 1. On the other hand, if $U=W_{0}$, then $\left|\mathcal{A}_{1}\right| \geq 2$ and $\left|\mathcal{A}_{1} \cap \mathcal{C}_{1}\right|=1$ imply that there is $V \in \mathcal{A}_{1}^{*} \cap \mathcal{C}_{0}$, and performing a type I swap between $y_{0} \mid W_{0}$ and some $y \mid V$ results (in view of Lemma 3.1.2) in a new product decomposition $W^{\prime}$ with $\tilde{\sigma}\left(W^{\prime}\right)=\widetilde{\sigma}(W), W_{0}^{\prime} \in \mathcal{C}_{0}, V^{\prime} \in \mathcal{C}_{1}, y_{0} \mid V^{\prime}$ and $W^{\prime}$ also satisfying the hypothesis of Case 1. Consequently, there are $W$ and $W^{\prime}$ satisfying the hypotheses of Case 1 with $\widetilde{\sigma}(W)=\widetilde{\sigma}\left(W^{\prime}\right)$ and w.l.o.g. $y_{0} \mid U \neq W_{0}$ and $y_{0} \mid U^{\prime}=W_{0}^{\prime}$ (thus $W^{\prime}$ is defined as in the second sentence of Case 1). Since $U \in \mathcal{C}_{1}$ and $\widetilde{\sigma}\left(W^{\prime}\right)=\widetilde{\sigma}(W)$ with $W_{0}^{\prime} \in \mathcal{C}_{1}$ (with $W^{\prime}$ as in the second sentence of Case 1), letting $\sigma\left(W_{0}\right)=C f_{1}+f_{2}$ we see that $a=(1-C) f_{1}-f_{2}$. 
Let $\mathcal{D}_{1}=\mathcal{A}_{2}^{*}\left(W^{\prime}\right) \cap \mathcal{C}_{1}\left(W^{\prime}\right)$ and $\mathcal{D}_{2}=\mathcal{A}_{2}^{*}\left(W^{\prime}\right) \cap \mathcal{C}_{0}\left(W^{\prime}\right)$. Since $\left|\mathcal{A}_{1} \cap \mathcal{C}_{1}\right|=1$ and $W_{0}^{\prime} \in \mathcal{C}_{1}$, we have $\left|\mathcal{D}_{1}\right|=m-2$, and by Claim A we have $\left|\mathcal{D}_{2}\right| \geq 1$ (else $e_{1}$ is a term with multiplicity at least $(m+1) n-2 \geq m n$, contradicting $S \in \mathcal{A}(G)$ ). If Lemma 5.2(ii) holds for $W^{\prime}$, then Lemmas 5.3.1 and 3.1.1 imply that $a=0$, a contradiction. Therefore Lemma 5.2(i) holds for $W^{\prime}$. Let $g$ and $\alpha$ be as given by Lemma 5.2(i).

Since $\left|\mathcal{D}_{2}\right| \geq 1$, let $V \in \mathcal{A}_{2}^{*}(W) \cap \mathcal{C}_{0}(W)$ (recall that no terms from $\mathcal{C}_{0}^{*}(W)$ were involved in the swap between $W$ and $W^{\prime}$, so $\left.V^{\prime}=V\right)$. If $\iota(V)=g^{n}$, then, performing type III swaps between $V$ and some $Z \in \mathcal{A}_{2}^{*} \cap \mathcal{C}_{1}$, and between $V$ and $W_{0}$, we conclude from Lemmas 3.1.2, 3.1.3 and 3.4.3 that $\psi(x)=\alpha$ for all $x \mid V$, whence $g e_{1}+e_{2}+\alpha$ has multiplicity at least $m n-1$ in $S$, as desired. Therefore, in view of $\iota\left(W_{0}^{(2)}\right) \equiv g^{n-1}(g+1) \bmod n$, we see that there exist $x \mid W_{0}^{(2)}=W_{0}^{\prime(2)}$ and $y \mid V=V^{\prime}$ such that $\epsilon^{\prime}(x, y) \notin\{1, n\}$. Hence, Lemmas 5.3 .3 (applied to $\left.W^{\prime}\right)$ and 3.12 yield $a= \pm\left(\left(1-C^{\prime}\right) f_{1}-f_{2}\right)$, where $\sigma(V)=C^{\prime} f_{1}+f_{2}$. Thus, since $a=(1-C) f_{1}-f_{2}$ and $m \geq 3$, we conclude that $C^{\prime} f_{1}=C f_{1}$ and $\sigma(V)=\sigma\left(W_{0}\right)$. As $V \in \mathcal{A}_{2}^{*}(W) \cap \mathcal{C}_{0}(W)$ was arbitrary, we see that $\sigma(V)=C f_{1}+f_{2}$ for all $V \in \mathcal{A}_{2}(W) \cap \mathcal{C}_{0}(W)$. On the other hand, if $Z \in \mathcal{A}_{1}(W) \cap \mathcal{C}_{0}(W)$, then, performing type I swaps between $U$ and $Z$, we conclude from Lemma 3.1.2 that $a=\left(1-C^{\prime \prime}\right) f_{1}-f_{2}$, where $\sigma(Z)=C^{\prime \prime} f_{1}+f_{2}$. Thus $a=(1-C) f_{1}-f_{2}$ implies that $C^{\prime \prime} f_{1}=C f_{1}$, and now $\sigma(Z)=C f_{1}+f_{2}$ for all $Z \in \mathcal{A}_{1}(W) \cap \mathcal{C}_{0}(W)$. Consequently, $\sigma(Z)=C f_{1}+f_{2}$ for all $Z \in \mathcal{C}_{0}(W)$, contradicting $\mathrm{h}(\widetilde{\sigma}(W))<m$. This completes Case 1.

CASE 2: $\Omega_{0}^{u} \neq \emptyset$ and $y_{0} \mid U$ for some $U \in \mathcal{A}_{1} \cap \mathcal{C}_{0}$. Recall that $\left|\mathcal{A}_{1} \cap \mathcal{C}_{1}\right|$ $\geq 1$ and $\left|\mathcal{A}_{1}\right| \geq 2$. Hence Case 3 of Claim $\mathrm{A}$ and the hypothesis of Case 2 further imply that $\left|\mathcal{A}_{1} \cap \mathcal{C}_{0}\right|=1$. Thus, if $U \neq W_{0}$, then $W_{0} \in \mathcal{C}_{1}$, and performing a type I swap between $y_{0} \mid U$ and some $y \mid W_{0}$ results (in view of Lemma 3.1.2) in a product decomposition $W^{\prime}$ with $y_{0} \mid W_{0}^{\prime}, W_{0}^{\prime} \in \mathcal{C}_{0}$, $\tilde{\sigma}\left(W^{\prime}\right)=\tilde{\sigma}(W)$ and $W^{\prime}$ satisfying the hypotheses of Case 2. Thus w.l.o.g. we may assume $U=W_{0}$.

Since $\left|\mathcal{A}_{1} \cap \mathcal{C}_{1}\right| \geq 1$, let $V \in \mathcal{A}_{1} \cap \mathcal{C}_{1}^{*}\left(\right.$ recall $W_{0}=U \in \mathcal{C}_{0}$, so $\mathcal{C}_{1}=\mathcal{C}_{1}^{*}$ ). Performing a type I swap between $y_{0} \mid W_{0}$ and some $y \mid V$, letting $W^{\prime}$ be the resulting product decomposition, we conclude from Lemma 3.1.2 that $a=(C-1) f_{1}+f_{2}$, where $\sigma\left(W_{0}\right)=C f_{1}+f_{2}$. Since $\left|\mathcal{A}_{1} \cap \mathcal{C}_{0}\right|=1$ and $W_{0} \in \mathcal{C}_{0}$, let w.l.o.g. $W_{1}, \ldots, W_{m-1}$ be the blocks of $\mathcal{A}_{2}^{*} \cap \mathcal{C}_{0}$. If $x \mid W_{0}^{(2)}$ and $y \mid W_{j}$, with $j \in[1, m-1]$ and $\iota(x)=\iota(y)$, then, performing a type III swap between $x \mid W_{0}$ and $y \mid W_{j}$ and between $x \mid W_{0}^{\prime}$ and $y \mid W_{j}^{\prime}$, we conclude in view of Lemmas 3.1.3 and 3.1.2 that $\psi(x)=\psi(y)$; thus, letting $\mathcal{D}_{1}=$ $\mathcal{A}_{2}^{*} \cap \mathcal{C}_{0}=\left\{W_{1}, \ldots, W_{m-1}\right\}$ and $\mathcal{D}_{2}=\mathcal{A}_{2}^{*} \cap \mathcal{C}_{1}$, we see that hypothesis (a) holds in Lemma 5.2. If Lemma 5.2(i) holds, then $g e_{1}+e_{2}+\alpha$ is a term of $S$ with multiplicity at least $m n-1$, as desired. Therefore Lemma 5.2(ii) 
holds, whence Lemmas 5.3 .2 and 3.1 .3 imply that $a \in\left\langle f_{1}\right\rangle$, contradicting $a=(C-1) f_{1}+f_{2}$. This completes Case 2 .

Note that if $\Omega_{0}^{u}=\emptyset$, then (in view of $\left|\mathcal{A}_{1}\right| \geq 2$ ) we may w.l.o.g. assume $y_{0} \mid U$ with $U \neq W_{0}$, by an appropriate type I swap. Moreover, when $\Omega_{0}^{u}=\emptyset$, we will w.l.o.g. assume $\widetilde{\sigma}(W)=f_{1}^{m-1} f_{2}^{m-1}\left(f_{1}+f_{2}\right)$ with $\mathcal{C}_{1}$ consisting of those blocks $W_{i}$ with $\sigma\left(W_{i}\right)=f_{1}$.

CASE 3: $\Omega_{0}^{u}=\emptyset$ and $y_{0} \mid U \in \mathcal{A}_{1}^{*}$ with $U \in \mathcal{C}_{0}$. We may w.l.o.g. assume $W_{0} \in \mathcal{C}_{1}$. Performing a type I swap between $y_{0} \mid U$ and some $y \mid W_{0}$, letting $W^{\prime}$ be the resulting product decomposition, we conclude from Lemma 3.3. 4 that $a=f_{2}$. Let $\mathcal{D}_{1}=\mathcal{A}_{2}^{*}\left(W^{\prime}\right) \cap \mathcal{C}_{2}\left(W^{\prime}\right)$ and let $\mathcal{D}_{2}=A_{2}^{*}\left(W^{\prime}\right) \cap \mathcal{C}_{1}\left(W^{\prime}\right)$. Observe that $\left|\mathcal{D}_{1}\right|=m-1$, else performing a type I swap between $y_{0} \mid U$ and some $V \in \mathcal{A}_{1} \cap \mathcal{C}_{2}$ would imply in view of Lemma 3.3.5 that $a=f_{1}$, contradicting $a=f_{2}$. If a type III swap between $W_{0}^{\prime}$ and some $W_{j}^{\prime} \in \mathcal{D}_{1}$ results in a new product decomposition $W^{\prime \prime}$ with $\sigma\left(W_{0}^{\prime \prime}\right) \neq \sigma\left(W_{0}^{\prime}\right)$, then Lemma 3.3 .5 implies $\sigma\left(W_{0}^{\prime \prime}\right)=f_{2}$, whence, performing a type I swap between $y_{0} \mid W_{0}^{\prime \prime(1)}=W_{0}^{\prime(1)}$ and $U^{\prime \prime}=U^{\prime}$, we conclude from Lemma 3.2 . 3 that $-a=f_{1}-f_{2}$, contradicting $a=f_{2}$. Thus hypothesis (a) of Lemma 5.2 holds for $W^{\prime}$. If Lemma 5.2(i) holds, then $g e_{1}+e_{2}+\alpha$ has multiplicity at least $m n-1$ in $S$, as desired. Therefore, Lemma 5.2(ii) holds, whence Lemmas 5.32 and 3.2 .5 imply that $a \in\left\langle f_{1}\right\rangle$, contradicting that $a=f_{2}$ and completing Case 3.

CASE 4: $\Omega_{0}^{u}=\emptyset$ and $y_{0} \mid U \in \mathcal{A}_{1}^{*}$ with $U \notin \mathcal{C}_{0}$. We may w.l.o.g. assume $U \in \mathcal{C}_{1}$. If $W_{0} \in \mathcal{C}_{1}$, then performing type I swaps between $W_{0}$ and $U$ would imply, in view of Lemma 3.3.1, that $a=0$, a contradiction. Moreover, this also shows that $\mathcal{A}_{1} \cap \mathcal{C}_{1}=\{U\}$.

Suppose $W_{0} \in \mathcal{C}_{2}$. Performing a type I swap between $y_{0} \mid U$ and some $y \mid W_{0}$, letting $W^{\prime}$ be the resulting product decomposition, we conclude from Lemma 3.2 .3 that $\widetilde{\sigma}\left(W^{\prime}\right)=\widetilde{\sigma}(W), W_{0}^{\prime} \in \mathcal{C}_{1}, a=f_{1}-f_{2}$ and $n e_{1}=$ $\sigma\left(U^{\prime}\right)=f_{2}$. Let $\mathcal{D}_{1}=\mathcal{A}_{2}^{*}\left(W^{\prime}\right) \cap \mathcal{C}_{1}\left(W^{\prime}\right)$ and let $\mathcal{D}_{2}=\mathcal{A}_{2}^{*}\left(W^{\prime}\right) \cap \mathcal{C}_{0}\left(W^{\prime}\right)$. Since $\mathcal{A}_{1} \cap \mathcal{C}_{1}=\{U\}$, we have $\left|\mathcal{D}_{1}\right|=m-2$. Since $n e_{1}=f_{2} \neq f_{1}+f_{2}$, we have $Z \in \mathcal{C}_{0}$ with $Z \in \mathcal{A}_{2}^{*}$, and thus $\left|\mathcal{D}_{2}\right| \geq 1$. Apply Lemma 5.2 to $W^{\prime}$. If Lemma 5.2(ii) holds, then Lemmas 5.3.1 and 3.2 .1 imply $\psi_{1}(a)=0$, contradicting $a=f_{1}-f_{2}$. Therefore Lemma 5.2 (i) holds, whence gne $e_{1}+$ $n e_{2}+n \alpha=\sigma(V)=f_{1}$, where $V \in \mathcal{D}_{1}$. If there is a type III swap between $Z^{\prime}=Z$ and $W_{0}^{\prime}$ resulting in a product decomposition $W^{\prime \prime}$ with $\sigma\left(W_{0}^{\prime \prime}\right) \neq \sigma\left(W_{0}^{\prime}\right)$, then Lemma 3.3. 4 implies that $\sigma\left(W_{0}^{\prime \prime}\right)=f_{1}+f_{2}$, whence, performing a type I swap between $y_{0} \mid W_{0}^{\prime \prime}$ and $y \mid U^{\prime \prime}=U^{\prime}$, we conclude from Lemma 3.3.5 that $-a=-f_{1}$, contradicting $a=f_{1}-f_{2}$. Therefore hypothesis (a) holds in Lemma 5.2 for $W^{\prime}$ with the roles of $\mathcal{D}_{1}$ and $\mathcal{D}_{2}$ reversed. Apply Lemma 5.2 in this case. If Lemma 5.2(ii) holds, then Lemmas 5.3 .2 and 3.24 imply that $a \in\left\langle f_{2}\right\rangle$, contradicting $a=f_{1}-f_{2}$. There- 
fore Lemma 5.2 (i) holds, whence gne $e_{1}+n e_{2}+n \alpha=\sigma(Z)=f_{1}+f_{2}$, contradicting gne $e_{1}+n e_{2}+n \alpha=f_{1}$. So we may assume instead that $W_{0} \in \mathcal{C}_{0}$.

Performing a type I swap between $y_{0} \mid U$ and some $y \mid W_{0}$, letting $W^{\prime}$ be the resulting product decomposition, we conclude from Lemma 3.3.4 that $\tilde{\sigma}\left(W^{\prime}\right)=\widetilde{\sigma}(W), W_{0}^{\prime} \in \mathcal{C}_{1}, a=-f_{2}$, and $n e_{1}=\sigma\left(U^{\prime}\right)=f_{1}+f_{2}$. Let $\mathcal{D}_{1}=\mathcal{A}_{2}^{*}\left(W^{\prime}\right) \cap \mathcal{C}_{2}\left(W^{\prime}\right)$. If there is $V \in \mathcal{A}_{1} \cap \mathcal{C}_{2}$, then, performing a type I swap between $y_{0} \mid U$ and some $y \mid V$, we conclude from Lemma 3.2. 3 that $a=f_{1}-f_{2}$, contradicting $a=-f_{2}$. Therefore $\left|\mathcal{D}_{1}\right|=m-1$. Let $\mathcal{D}_{2}=$ $\mathcal{A}_{2}^{*}\left(W^{\prime}\right) \cap \mathcal{C}_{1}\left(W^{\prime}\right)$. Since $\mathcal{A}_{1} \cap \mathcal{C}_{1}=\{U\}$, we have $\left|\mathcal{D}_{2}\right| \geq m-2$. Thus we may apply Lemma 5.2 to $W^{\prime}$. If Lemma 5.2(i) holds, then $g e_{1}+e_{2}+\alpha$ is a term of $S$ with multiplicity at least $m n-1$, as desired. Therefore Lemma 5.2(ii) holds, whence Lemmas 5.3 .3 and 3.2 .3 imply that $a= \pm\left(f_{1}-f_{2}\right)$, contradicting $a=-f_{2}$. This completes Case 4 .

By Claim B, we now have $S_{1}=e_{1}^{\left|S_{1}\right|}$. From Lemma 2.3.3 and $e_{1} \mid S_{1}$, we have $\operatorname{ord}\left(e_{1}\right)=m n$. Hence there exists $e_{2}^{\prime \prime} \in e_{2}+n G$ such that $\left(e_{1}, e_{2}^{\prime \prime}\right)$ is a basis for $G$; we provide a short sketch of how to choose such an $e_{2}^{\prime \prime}$ below.

Take a basis $\left(e_{1}, e_{2}^{\prime}\right)$. Write $e_{2}=a e_{1}+b e_{2}^{\prime}$, where $a, b \in \mathbb{Z}$. Let $m^{\prime} \mid m$ be maximal such that $\operatorname{gcd}\left(m^{\prime}, n\right)=1$ (and thus every prime dividing $m^{\prime-1} m n$ also divides $n)$. Note that $\left(m e_{1}, m e_{2}\right)$ being a basis in $\varphi(G) \operatorname{implies} \operatorname{gcd}(b, n)$ $=1$. Since $\operatorname{gcd}\left(m^{\prime}, n\right)=1$, use the Chinese Remainder Theorem, for each prime $p \mid m^{\prime}$, to find $x \in \mathbb{Z}$ such that $p \mid b+n x$ for all primes $p \mid m^{\prime}$, and thus $\operatorname{gcd}\left(b+n(x+1), m^{\prime}\right)=1$. Now let $e_{2}^{\prime \prime}=e_{2}+n(x+1) e_{2}^{\prime}$.

Thus, after changing notation if necessary (exchanging $e_{2}$ for $e_{2}^{\prime \prime}$ ), we may suppose that $\left(e_{1}, e_{2}\right)$ is a basis of $G$. If $g \in G$ and $x, y \in \mathbb{Z}$ with $g=x e_{1}+y e_{2}$, then we set $\pi_{1}(g)=x e_{1}$ and $\pi_{2}(g)=y e_{2}$. Note that we now have $\sigma(\psi(R))=0$ in any type II swap and that $\left(f_{1}, f_{2}\right)$ and $\left(n e_{1}, n e_{2}\right)$ are now two (possibly) distinct bases of $\operatorname{Ker}(\varphi) \cong C_{m} \oplus C_{m}$.

Claim C. There exists $x_{0} \mid S_{2}$ such that $x-y \in\left\langle e_{1}\right\rangle$ for all $x y \mid x_{0}^{-1} S_{2}$.

Proof. We need to show that there exists $x_{0} \mid S_{2}$ such that $\pi_{2}(\psi(x))=$ $\pi_{2}(\psi(y))$ for all $x y \mid x_{0}^{-1} S_{2}$. We divide the proof into four cases.

CASE 1: $\Omega_{0}^{u} \neq \emptyset$ and there is $U \in \mathcal{A}_{1}^{*} \cap \mathcal{C}_{1}$. In this case, we have

$$
n e_{1}=\sigma(U)=f_{1} .
$$

Let $V \in \mathcal{A}_{2}^{*}$. Perform type (II) swaps between $W_{0}$ and $V$. If $V, W_{0} \in \mathcal{C}_{1}$, then we conclude from Lemmas 3.1.1 and 3.4.1 that $\pi_{2}(\psi(x))=\alpha_{2}$ (say) for all $x \mid V W_{0}^{(2)}$. If $V, W_{0} \in \mathcal{C}_{0}$, then we conclude from Lemmas 3.1 .3 and 3.41 and 19 that $\psi_{2}$ is constant on $V W_{0}^{(2)}$, whence 19 further implies that $\pi_{2}(\psi(x))=\alpha_{2}$ for all $x \mid V W_{0}^{(2)}$. If $\left|\left\{V, W_{0}\right\} \cap \mathcal{C}_{1}\right|=1$, then we conclude from Lemmas 3.1 .2 and 3.4 . 3 that $\pi_{2}(\psi(x))=\alpha_{2}$ for all $x \mid x_{0}^{-1} V W_{0}^{(2)}$, for 
some $x_{0} \mid V W_{0}^{(2)}$. If $\pi_{2}\left(\psi\left(x_{0}\right)\right) \neq \alpha_{2}$ and $x_{0} \mid V$, then pull $x_{0}$ up into a new product decomposition $W^{\prime}$ and assume we began with $W^{\prime}$ instead of $W$ (note that $(19)$ holds independent of $W^{\prime}$ and that $\widetilde{\sigma}(W)=\widetilde{\sigma}\left(W^{\prime}\right)$ follows by Lemma 3.1.2, so all previous arguments can be applied to $W^{\prime}$ regardless of whether $\mathcal{A}_{1}^{*}\left(W^{\prime}\right) \cap \mathcal{C}_{1}\left(W^{\prime}\right)$ is nonempty or not). Doing these swaps for all $V \in \mathcal{A}_{2}^{*}$, we conclude that there is an $x_{0} \mid S_{2}$ such that $\pi_{2}(\psi(x))=\alpha_{2}$ for all $x \mid x_{0}^{-1} S_{2}$, completing Case 1 .

CASe 2: $\Omega_{0}^{u} \neq \emptyset$ and $\mathcal{A}_{1} \cap \mathcal{C}_{1}=\left\{W_{0}\right\}$. Performing type II swaps between $W_{0}$ and each $U \in \mathcal{A}_{2}^{*} \cap \mathcal{C}_{1}$, we conclude from Lemmas 3.4.1 and 3.1. 1 that $\pi_{2}(\psi(x))=\alpha_{2}$ (say) for all $x \mid W_{0}^{(2)} U$, with $U \in \mathcal{A}_{2}^{*} \cap \mathcal{C}_{1}$. Let w.l.o.g. $W_{1}, \ldots, W_{l}$ be the blocks in $\mathcal{A}_{2} \cap \mathcal{C}_{0}$, and let $W_{m+1}, \ldots, W_{2 m-2}$ be the blocks in $\mathcal{A}_{2}^{*} \cap \mathcal{C}_{1}$. Note $l \geq 1$, else Claim $\mathrm{C}$ follows by the previous conclusion. Performing type II swaps between $W_{0}$ and $W_{j}$, with $j \in[1, l]$, we conclude from Lemmas 3.43 and 3.1 .2 that $\pi_{2}(\psi(x))=\alpha_{2}$ for all $x \mid z_{j}^{-1} W_{j}$, for some $z_{j} \mid W_{j}$. We may w.l.o.g. assume $\pi_{2}\left(\psi\left(z_{j}\right)\right) \neq \alpha_{2}$ for $j \in\left[1, l^{\prime}\right]$ and $\pi_{2}\left(\psi\left(z_{j}\right)\right)=\alpha_{2}$ for $j \in\left[l^{\prime}+1, l\right]$. We have $l^{\prime} \geq 2$, else Claim $\mathrm{C}$ follows.

Perform a type II swap between $z_{1} \mid W_{1}$ and any term $y \mid W_{0}^{(2)}$, and let $W^{\prime}$ denote the resulting product decomposition. Since $\pi_{2}\left(\psi\left(z_{1}\right)\right) \neq \alpha_{2}=$ $\pi_{2}(\psi(y))$, we know that $\pi_{2}\left(\sigma\left(W_{0}\right)\right) \neq \pi_{2}\left(\sigma\left(W_{0}^{\prime}\right)\right)$, and hence $\sigma\left(W_{0}\right) \neq \sigma\left(W_{0}^{\prime}\right)$. Thus Lemma 3.1.2 implies that $\widetilde{\sigma}\left(W^{\prime}\right)=\widetilde{\sigma}(W), W_{0}^{\prime} \in \mathcal{C}_{0}$ and $W_{1}^{\prime} \in \mathcal{C}_{1}$.

Now pull the term $z_{2} \mid W_{2}$ up into a new product decomposition $W^{\prime \prime}$. Note by Lemma 3.12 that $\widetilde{\sigma}\left(W^{\prime \prime}\right)=\widetilde{\sigma}(W)$. If $W_{0}^{\prime \prime} \in \mathcal{C}_{1}$, then the arguments of the first paragraph show that $\pi_{2}\left(\psi\left(z_{2}\right)\right)=\alpha_{2}$, contradicting $l^{\prime} \geq 2$. Therefore $W_{2}^{\prime \prime} \in \mathcal{C}_{1}$ instead. However, noting that $y W_{0}^{(1)} \mid W_{0}^{\prime \prime}$ for some $y \mid W_{0}^{(2)}$ (since $\sigma\left(\iota\left(W_{0}^{(2)}\right)\right) \equiv 1 \bmod n$ and $\left.\sigma\left(\iota\left(W_{2}^{\prime}\right)\right) \equiv 0 \bmod n\right)$, we can still perform the swap between $y \mid W_{0}^{\prime \prime}$ and $z_{1} \mid W_{1}^{\prime \prime}=W_{1}$ described in the previous paragraph, which results in a new product decomposition $W^{\prime \prime \prime}$ in which the $m$ blocks

$$
W_{1}^{\prime \prime \prime}=W_{1}^{\prime}, W_{2}^{\prime \prime \prime}=W_{2}^{\prime \prime}, W_{m+1}^{\prime \prime \prime}=W_{m+1}, \ldots, W_{2 m-2}^{\prime \prime \prime}=W_{2 m-2}
$$

all have equal sum $f_{1}$, contradicting $S \in \mathcal{A}(G)$ and completing Case 2 .

CASE 3: Either $\left(\Omega_{0}^{u} \neq \emptyset\right.$ and $\left.\mathcal{A}_{1} \cap \mathcal{C}_{1}=\emptyset\right)$ or $\left(\Omega_{0}^{u}=\emptyset\right.$ and $\left.W_{0} \notin \mathcal{C}_{0}\right)$. If $\Omega_{0}^{u}=\emptyset$, we may w.l.o.g. assume $\widetilde{\sigma}(W)=f_{1}^{m-1} f_{2}^{m-1}\left(f_{1}+f_{2}\right)$ with $\mathcal{C}_{1}$ those blocks with sum $f_{1}$ and $\mathcal{C}_{2}$ those blocks with sum $f_{2}$, and that $W_{0} \in \mathcal{C}_{2}$. Let w.l.o.g. $W_{1}, \ldots, W_{s}$ be the $s \leq m-1$ blocks of $\mathcal{C}_{1} \cap \mathcal{A}_{2}^{*}$. Let $\sigma\left(W_{0}\right)=C f_{1}+f_{2}$ and $F=(C-1) f_{1}+f_{2}$. If $\Omega_{0}^{u} \neq \emptyset$, then we have $s=m-1$ by hypothesis. If $s=0$, then $\left|\mathcal{A}_{1}^{*} \cap \mathcal{C}_{1}\right|=m-1$ (recall $W_{0} \in \mathcal{C}_{2}$ ), implying $e_{1}$ is a term with multiplicity at least $m n-1$ in $S$ (in view of Claim B), as desired. Therefore we may assume $s>0$.

We claim, for any $W$ satisfying the hypothesis of Case 3 and notated as above, in particular, with $W_{1}, \ldots, W_{s}$ being the blocks of $\mathcal{C}_{1} \cap \mathcal{A}_{2}^{*}$ (and in 
fact, if $W \in \Omega_{0}^{n u}$, we will not need that $\left.\Omega_{0}^{u}=\emptyset\right)$, that

$$
\pi_{2}\left(\psi\left(x_{0}^{-1} W_{0}^{(2)} \prod_{\nu=1}^{s} W_{\nu}\right)\right)=q_{2}^{(s+1) n-1}
$$

for some $x_{0} \mid W_{0}^{(2)} \prod_{\nu=1}^{s} W_{\nu}$ and $q_{2} \in \operatorname{Ker}(\varphi)$. To show this, perform type II swaps between $W_{0}$ and $W_{i}, i \in[1, s]$. If $\pi_{2}(F)=0$, then Lemmas 3.4 .1 and either 3.1.2 or 3.2 .3 imply that 200 holds with $\pi_{2}\left(x_{0}\right)=q_{2}$ as well. If $\pi_{2}(F) \neq 0$ and 20 fails, then Lemmas 3.4 .3 and either 3.1 .2 or 3.2 .3 imply that $\pi_{2}(\psi(z))=q_{2}$ (say) for all $z \mid x_{i}^{-1} W_{0}^{(2)} W_{i}$, for some $x_{i} \mid W_{i}, i \in[1, s]$; moreover, $s \geq 2$ and w.l.o.g. $\pi_{2}\left(\psi\left(x_{1}\right)\right)$ and $\pi_{2}\left(\psi\left(x_{2}\right)\right)$ are not equal to $q_{2}$. Pull $x_{1} \mid W_{1}$ up into a new product decomposition $W^{\prime}$. If $\sigma\left(W_{0}^{\prime}\right)=\sigma\left(W_{0}\right)$, then the arguments of the previous sentence imply either $\pi_{2}\left(\psi\left(x_{1}\right)\right)=q_{2}$ or $\pi_{2}\left(\psi\left(x_{2}\right)\right)=q_{2}$, a contradiction. If $\sigma\left(W_{0}^{\prime}\right) \neq \sigma\left(W_{0}\right)$ and $W \in \Omega_{0}^{u}$, then Lemma 3.12 implies that $W^{\prime} \in \Omega_{0}^{u}$ with $W_{0}^{\prime} \in \mathcal{C}_{1}$, whence Claim $\mathrm{C}$ follows in view of Case 2 applied to $W^{\prime}$. Therefore we may assume $\sigma\left(W_{0}^{\prime}\right) \neq \sigma\left(W_{0}\right)$, $W \in \Omega_{0}^{n u}$ and $W_{0}^{\prime} \in \mathcal{C}_{1}$ (in view of Lemma 3.2.3). Let $y$ be a term that divides both $W_{0}^{(2)}$ and $W_{0}^{\prime(2)}$ (possible since $\sigma\left(\iota\left(W_{0}\right)\right) \equiv 1 \bmod n$ ). Choose $I$ such that $\min I \equiv \iota(y) \bmod n$, and consequently $\epsilon(y, z)=0$ for any $z$ (in view of (13)). Note that while the new choice of $I$ may change the overall value of $\psi(x)$, where $x \mid S_{2}$, in a nontrivial manner, nonetheless, the value of $\pi_{2}(\psi(x))$ remains unchanged. Perform type II swaps between $y \mid W_{0}$ and any $z \mid W_{2}$. In view of our choice of $I$, Lemma 3.2.3 and $\pi_{2}\left(\psi\left(x_{2}\right)\right) \neq$ $q_{2}=\pi_{2}(\psi(y))$, we first conclude that $-\psi\left(x_{2}\right)+\psi(y)=F=-f_{1}+f_{2}$ (since $-\pi_{2}\left(\psi\left(x_{2}\right)\right)+\pi_{2}(\psi(y)) \neq 0$, implying $-\psi\left(x_{2}\right)+\psi(y) \neq 0$, and since $\epsilon(y, z)=0)$, implying $\pi_{2}(F) \neq 0$, and then that $-\psi(z)+\psi(y)=0$ if $z \neq x_{2}$ (since $-\pi_{2}(\psi(z))+\pi_{2}(\psi(y))=0 \neq \pi_{2}(F)$ ); in particular, $\psi_{1}\left(x_{2}\right) \neq \psi_{1}(z)$ for $z \mid x_{2}^{-1} W_{2}$. However, performing type II swaps between $y \mid W_{0}^{\prime}$ and any $z \mid W_{2}^{\prime}=W_{2}$, we conclude from Lemma 3.2.1 and the choice of $I$ that $\psi_{1}$ is constant on $W_{2}^{\prime}=W_{2}$, contradicting the previous sentence. Thus 20) is established in all cases.

Next we proceed to show that $s=m-1$. To this end, suppose $s<m-1$. As noted before, we may then assume $\Omega_{0}^{u}=\emptyset$. Let $U \in \mathcal{A}_{1}^{*} \cap \mathcal{C}_{1}$ (which is nonempty by the assumption $s<m-1$ ). Then $f_{1}=\sigma(U)=n e_{1}$. Let $x_{0}$ and $q_{2}$ be as defined by (20). Thus, performing type II swaps between a fixed $x_{1} \mid x_{0}^{-1} W_{0}^{(2)}$ and any $y \mid V \in \mathcal{A}_{2}^{*} \cap\left(\mathcal{C}_{2} \cup \mathcal{C}_{0}\right)$, we conclude from $f_{1}=$ $\sigma(U)=n e_{1}$ and Lemmas 3.2 . 2 and 3.2 .5 that $\psi_{2}(V)=\psi_{2}\left(x_{1}\right)^{n}$ for all such blocks $V \in \mathcal{A}_{1}^{*} \cap\left(\mathcal{C}_{2} \cup \mathcal{C}_{0}\right)$. Hence, in view of $n e_{1}=f_{1}$, we conclude that $\pi_{2}(\psi(V))=\pi_{2}\left(\psi\left(x_{1}\right)\right)^{n}=q_{2}^{n}$ for all such $V$, which combined with 20 implies Claim C. So we may assume $s=m-1$.

In the case $W \in \Omega_{0}^{n u}$, we have assumed $\Omega_{0}^{u}=\emptyset$. However, we will temporarily drop this assumption, allowing consideration of $W \in \Omega_{0}^{n u}$ even 
when $\Omega_{0}^{u} \neq \emptyset$, provided it still satisfies the hypothesis of Case 3 and follows the notation given in the first paragraph with $s=m-1$. This will extend until the end of assertion A1 below, which shows that the exceptional term $x_{0}$ in 20 is not necessary.

A1. For every $W \in \Omega_{0}$ satisfying the hypotheses of Case 3 (allowing $W \in \Omega_{0}^{n u}$ even if $\left.\Omega_{0}^{u} \neq \emptyset\right)$, we have $\pi_{2}\left(\psi\left(x_{0}\right)\right)=q_{2}$, where $q_{2}$ and $x_{0}$ are as given by (20) (and $W$ is notated using the conventions from the start of Case 3$)$.

Proof of A1. Assume instead there exists $W \in \Omega_{0}$ satisfying the hypotheses of Case 3 with $\pi_{2}\left(\psi\left(x_{0}\right)\right) \neq q_{2}$.

Suppose $x_{0} \mid W_{j}$ with $j>0$. Pull up an arbitrary $y \mid W_{k} \in \mathcal{A}_{2}$, with $k \geq m$, into a resulting product decomposition $W^{\prime \prime}$ (such a block exists, else (20) completes Claim C). If $W^{\prime \prime}$ satisfies the hypotheses of Case 3, then applying (20) to $W^{\prime \prime}$ we conclude that $\pi_{2}(\psi(y))=q_{2}$ (since $x_{0} \mid W_{j}$ with $j>0$ ), whence Claim $\mathrm{C}$ follows in view of 20 and the arbitrariness of $y$. Therefore we may instead assume $W^{\prime \prime}$ does not satisfy the hypotheses of Case 3, whence, in view of Cases 1 and 2, we may assume $W^{\prime \prime} \in \Omega_{0}^{n u}$ with $W_{0}^{\prime \prime} \in \mathcal{C}_{0}\left(W^{\prime \prime}\right)$.

Let $z$ be a term dividing both $W_{0}^{(2)}$ and $W_{0}^{\prime \prime(2)}$ (which exists in view of $\left.\sigma\left(\iota\left(W_{0}^{(2)}\right)\right) \equiv 1 \bmod n\right)$. Note that we cannot have $0=\psi(z)-\psi\left(x_{0}\right)+$ $\epsilon\left(z, x_{0}\right) n e_{1}$, as then $0=\pi_{2}\left(\psi\left(x_{0}\right)\right)-\pi_{2}(\psi(z))=\pi_{2}\left(\psi\left(x_{0}\right)\right)-q_{2}$, a contradiction to $\pi_{2}\left(\psi\left(x_{0}\right)\right) \neq q_{2}$. Thus, in view of 20) and Lemma 3.2.3 or 3.1. 2, it follows that performing a type II swap between $x_{0} \mid W_{j}$ and $z \mid W_{0}^{(2)}$ results in a new product decomposition $W^{\prime}$ in which $\sigma\left(W_{j}^{\prime}\right)=C f_{1}+f_{2}$ and $\sigma\left(W_{0}^{\prime}\right)=f_{1}$. Thus, if $W \in \Omega_{0}^{u}$, then we can apply Lemma 5.1 to conclude $W^{\prime \prime} \in \Omega_{0}^{u}$, contrary to the conclusion of the previous paragraph. Therefore we may assume $W \in \Omega_{0}^{n u}$. Hence, from $W^{\prime \prime} \in \Omega_{0}^{n u}$ and Lemma 3.3 , it follows that $\widetilde{\sigma}\left(W^{\prime \prime}\right)=\tilde{\sigma}(W)$, whence $\sigma\left(W_{0}^{\prime \prime}\right)=f_{1}+f_{2}$ (in view of $\left.W_{0}^{\prime \prime} \in \mathcal{C}_{0}\left(W^{\prime \prime}\right)\right)$. However, since $z \mid W_{0}^{\prime \prime}{ }^{(2)}$, we may still apply the previously described swap between $x_{0} \mid W_{j}^{\prime \prime}=W_{j}$ and $z \mid W_{0}^{\prime \prime}$ now in $W^{\prime \prime} \in \Omega_{0}^{n u}$, which results in a product decomposition $W^{\prime \prime \prime} \in \Omega^{\prime}$ with $v_{f_{2}}\left(\widetilde{\sigma}\left(W^{\prime \prime \prime}\right)\right)=m$ (as $\sigma\left(W_{j}^{\prime \prime \prime}\right)=\sigma\left(W_{j}^{\prime}\right)=C f_{1}+f_{2}=f_{2}$ and $\sigma\left(W_{j}^{\prime \prime}\right)=\sigma\left(W_{j}\right)=f_{1}$ and $\left.W_{0}^{\prime \prime} \in \mathcal{C}_{0}\right)$, contradicting $S \in \mathcal{A}(G)$. So we may assume $x_{0} \mid W_{0}$.

Perform a type II swap between an arbitrary $x \mid W_{0}^{(2)}$ and $y \mid W_{j}$ with $j \in[1, m-1]$. In view of Lemma 3.12 or 3.2 , 3 , it follows that

$$
\epsilon(x, y) n e_{1}+\psi(x)-\psi(y) \in\{0, F\} .
$$

If $x=x_{0}$, then it follows, in view of $\pi_{2}\left(\psi\left(x_{0}\right)\right)-\pi_{2}(\psi(y))=\pi_{2}\left(\psi\left(x_{0}\right)\right)-q_{2}$ $\neq 0$ and (21), that $\epsilon\left(x_{0}, y\right) n e_{1}+\psi\left(x_{0}\right)-\psi(y)=F$, and thus

$$
0 \neq \pi_{2}\left(\psi\left(x_{0}\right)\right)-q_{2}=\pi_{2}\left(\psi\left(x_{0}\right)\right)-\pi_{2}(\psi(y))=\pi_{2}(F) .
$$


Consequently, if $x \neq x_{0}$, then, from $\pi_{2}(\psi(x))-\pi_{2}(\psi(y))=q_{2}-q_{2}=0$ (in view of (20) and (21) and (22), it follows that

$$
\epsilon(x, y) n e_{1}+\psi(x)-\psi(y)=0 .
$$

As $y \mid W_{j}$ with $j \in[1, m-1]$ and $x \mid x_{0}^{-1} W_{0}^{(2)}$ were arbitrary above, we see that we can apply Lemma 5.4 with $i=0, Z=x_{0}^{-1} W_{0}^{(2)}$ and $\mathcal{D}=$ $\left\{W_{1}, \ldots, W_{m-1}\right\}$.

Thus we can choose $I$ appropriately so that, for some $q \in \operatorname{Ker}(\varphi)$,

$$
\psi(x)=q
$$

for all $x \mid x_{0}^{-1} W_{0}^{(2)} \prod_{\nu=1}^{m-1} W_{\nu}$, and

$$
\iota(x) \leq \iota(y)
$$

for all $x \mid x_{0}^{-1} W_{0}^{(2)}$ and $y \mid W_{i}, i \in[1, m-1]$. By performing a type II swap between $x_{0} \mid W_{0}$ and each $y \mid W_{i}$ with $i \in[1, m-1]$, we conclude, from $\pi_{2}\left(\psi\left(x_{0}\right)\right) \neq q_{2}=\pi_{2}(q)$ and either Lemma 3.1 .2 or 3.2 .3 , that

$$
\psi\left(x_{0}\right)-q+\epsilon\left(x_{0}, y\right) n e_{1}=(C-1) f_{1}+f_{2} .
$$

Thus $\epsilon\left(x_{0}, y\right)$ must be the same for every $y \mid W_{j}$ with $j \in[1, m-1]$. As a result, it follows in view of 13 that either $\iota\left(x_{0}\right) \leq \min \left(\operatorname{supp}\left(\iota\left(\prod_{\nu=1}^{m-1} W_{\nu}\right)\right)\right)$ or $\iota\left(x_{0}\right)>\max \left(\operatorname{supp}\left(\iota\left(\prod_{\nu=1}^{m-1} W_{\nu}\right)\right)\right)$. In the latter case, we may choose $I$ such that $\min I \equiv \iota\left(x_{0}\right) \bmod n$, and thus, in both cases (in view of (24)),

$$
\iota(x) \leq \iota(y)
$$

for all $x \mid W_{0}^{(2)}$ and $y \mid W_{i}, i \in[1, m-1]$, with 23 still holding for some $q \in \operatorname{Ker}(\varphi)$ (since (26) was all that was required in the proof of Lemma 5.4 to ensure (23) held). Consequently, (25) and (13) imply that

$$
\psi\left(x_{0}\right)=q+F=q+(C-1) f_{1}+f_{2} .
$$

Let $y \mid W_{k} \in \mathcal{A}_{2}$ with $k \geq m$ and $\pi_{2}(\psi(y)) \neq q_{2}$; such a term and block exists, else Claim C follows in view of (20). If $y \mid W_{k}$ could be pulled up into a new product decomposition $W^{\prime}$ with $x_{0} \mid W_{0}^{\prime}$, then $W^{\prime}$ must still satisfy the hypothesis of Case 3 (by the same arguments used when $x_{0} \mid W_{j}$ with $j>0$ ), whence applying (20) to $W^{\prime}$ implies $\pi_{2}\left(\psi\left(x_{0}\right)\right)=q_{2}$ or $\pi_{2}(\psi(y))=q_{2}$, contrary to our assumption. Therefore we may assume this is not the case, whence Theorem 2.6.2 implies that

$$
\iota\left(W_{0}^{(2)}\right)=g_{1}^{l} g_{2}^{n-1-l} \iota\left(x_{0}\right) \quad \text { and } \quad \iota\left(W_{k}\right)=g_{1}^{n-1-l} g_{2}^{l} \iota(y)
$$

for some $g_{1}, g_{2} \in \mathbb{Z}$ with $\operatorname{gcd}\left(g_{1}-g_{2}, n\right)=1$. If there existed $x_{0}^{\prime} \mid x_{0}^{-1} W_{0}^{(2)}$ such that $\epsilon\left(x_{0}^{\prime}, z\right)=\epsilon\left(x_{0}, z\right)$ for some $z \mid W_{k}$, then we could apply a type II swap between $z \mid W_{k}$ and each of $x_{0} \mid W_{0}$ and $x_{0}^{\prime} \mid W_{0}$, which in view of 
Lemma 3.1 .3 or Lemma 3.2 would imply that $\psi_{2}\left(x_{0}\right)=\psi_{2}\left(x_{0}^{\prime}\right)=\psi_{2}(q)$, contradicting (27). Therefore we may assume otherwise, whence (13) implies either

$$
\begin{aligned}
\iota\left(x_{0}\right) & \leq \min \left(\operatorname{supp}\left(\iota\left(W_{k}\right)\right)\right) \leq \max \left(\operatorname{supp}\left(\iota\left(W_{k}\right)\right)\right) \\
& <\min \left(\operatorname{supp}\left(\iota\left(x_{0}^{-1} W_{0}^{(2)}\right)\right)\right)
\end{aligned}
$$

or

$$
\begin{aligned}
\iota\left(x_{0}\right) & >\max \left(\operatorname{supp}\left(\iota\left(W_{k}\right)\right)\right) \geq \min \left(\operatorname{supp}\left(\iota\left(W_{k}\right)\right)\right) \\
& \geq \max \left(\operatorname{supp}\left(\iota\left(x_{0}^{-1} W_{0}^{(2)}\right)\right)\right) .
\end{aligned}
$$

In either case, we see that $\left|\operatorname{supp}\left(\iota\left(W_{k}\right)\right) \cap \operatorname{supp}\left(\iota\left(W_{0}^{(2)}\right)\right)\right| \leq 1$. As a result, (28) implies that w.l.o.g. $l=n-1, \iota\left(W_{0}^{(2)}\right)=g_{1}^{n-1} \iota\left(x_{0}\right)$ and $\iota\left(W_{k}\right)=$ $g_{2}^{n-1} \iota(y)$. Thus $\sigma\left(\iota\left(W_{k}\right)\right) \equiv 0 \bmod n$ and $\sigma\left(\iota\left(W_{0}^{(2)}\right)\right) \equiv 1 \bmod n$ imply that $\iota\left(W_{k}\right)=g_{2}^{n}$ and $\iota\left(x_{0}\right) \equiv g_{1}+1 \bmod n$.

If $(29)$ holds, then from $\iota\left(x_{0}\right) \equiv g_{1}+1 \bmod n$ and $(29)$ it follows that $\max I=g_{1}$. However, in view of (26), this is only possible if $\iota(x)=g_{1}$ for all $x \mid x_{0}^{-1} W_{0}^{(2)} \prod_{\nu=1}^{m-1} W_{\nu}$, in which case, since $\psi(x)=q$ also holds for all such terms (in view of (23)), it follows that $S$ contains a term with multiplicity $m n-1$, as desired. Therefore we can instead assume (30) holds. In this case, it follows, in view of 30$), \iota\left(x_{0}^{-1} W_{0}^{(2)}\right)=g_{1}^{n-1}$ and $\iota\left(x_{0}\right) \equiv g_{1}+1 \bmod n$, that

$$
\left\{g_{2}\right\}=\operatorname{supp}\left(\iota\left(W_{k}\right)\right)=\operatorname{supp}\left(\iota\left(x_{0}^{-1} W_{0}^{(2)}\right)\right)=\left\{g_{1}\right\},
$$

contradicting $\operatorname{gcd}\left(g_{1}-g_{2}, n\right)=1$.

We now return to arguments where we assume $\Omega_{0}^{u}=\emptyset$ when $W \in \Omega_{0}^{n u}$. In view of $\mathbf{A} 1$, we may assume $\pi_{2}(\psi(x))=q_{2}$ for all $x \mid W_{0}^{(2)} \prod_{\nu=1}^{m-1} W_{\nu}$. Let $y \mid W_{k}$ be arbitrary with $W_{k} \in \mathcal{A}_{2}$ and $k \geq m$. If we can pull up $y$ into a new product decomposition $W^{\prime}$ such that either $W^{\prime} \in \Omega_{0}^{u}$, or else $W^{\prime} \in \Omega_{0}^{n u}$ and $W_{0}^{\prime} \notin \mathcal{C}_{0}\left(W^{\prime}\right)$, then it follows, in view of Cases 1 and 2, $\mathbf{A} \mathbf{1}$ and $(20)$, that we may assume $\pi_{2}(\psi(y))=q_{2}$ also (note this is where we need that $W \in \Omega_{0}^{n u}$ is allowed in $\mathbf{A} \mathbf{1}$ even when $\left.\Omega_{0}^{u} \neq \emptyset\right)$. However, this can only fail if (by an appropriate choice for $f_{2}$ in the case when $W \in \Omega_{0}^{u}$ ) w.l.o.g.

$$
\tilde{\sigma}(W)=f_{1}^{m-1} f_{2}^{m-2}\left(C f_{1}+f_{2}\right)\left((1-C) f_{1}+f_{2}\right),
$$

with $\sigma\left(W_{k}\right)=(1-C) f_{1}+f_{2}$ and (recall) $\sigma\left(W_{0}\right)=C f_{1}+f_{2}$. Consequently, we see that there is at most one block $W_{k}$ for which this can fail (as $W_{0} \notin \mathcal{C}_{0}$ when $\left.\Omega_{0}^{u}=\emptyset\right)$. As Claim $\mathrm{C}$ follows otherwise, we may assume $W_{k} \in \mathcal{A}_{2}$ exists and that $\tilde{\sigma}(W)$ is of such form, and w.l.o.g. assume $k=2 m-2$. Now

$$
\begin{aligned}
C f_{1}+f_{2} & =\sigma\left(W_{0}\right)=Y_{1} n e_{1}+n e_{2}+n q_{2}, \\
f_{1} & =\sigma\left(W_{1}\right)=Y_{2} n e_{1}+n e_{2}+n q_{2},
\end{aligned}
$$


for some $Y_{i} \in \mathbb{Z}$. From $(32)$ and (33), we conclude that

$$
(C-1) f_{1}+f_{2} \in\left\langle n e_{1}\right\rangle .
$$

If there exists $U \in \mathcal{A}_{1}^{*}$, then $n e_{1}=\sigma(U)=f_{2}$ (in view of (31), $s=m-1$ and $\left.W_{k}=W_{2 m-2} \in \mathcal{A}_{2}\right)$; thus from (34) it follows that $(C-1) f_{1} \in\left\langle f_{2}\right\rangle$, which is only possible if $C \equiv 1 \bmod m$, contradicting $W \notin \mathcal{C}_{0}$ when $W \in \Omega_{0}^{n u}$ (in view of (31)). So we may instead assume $\left|\mathcal{A}_{1}\right|=1$. This same argument also shows that $\psi_{1}\left(n e_{1}\right) \neq 0$. Let $\mathcal{D}=\left\{W_{m}, \ldots, W_{2 m-2}\right\}$.

If $\psi_{2}\left(n e_{1}\right)=0$, then $n e_{1} \in\left\langle f_{1}\right\rangle$, which combined with (34) yields a contradiction to $\left(f_{1}, f_{2}\right)$ being a basis. Therefore $\psi_{2}\left(n e_{1}\right) \neq 0$. Thus, in view of Lemma 3.1.3 or Lemmas 3.2 .5 and 3.2 , 2, it follows that we may apply Lemma 5.4 with $Z=W_{0}^{(2)}, i=2$ and $\mathcal{D}$ as given above. Choose $I$ as in Lemma 5.4 (as mentioned before, changing $I$ does not affect the value of $\pi_{2}(\psi(x))$, and thus (20) remains unaffected). Then

$$
\psi_{2}(x)=\alpha_{2}
$$

for all $x \mid W_{0}^{(2)} \prod_{\nu=m}^{2 m-2} W_{\nu}$ and some $\alpha_{2} \in\left\langle f_{2}\right\rangle$, and

$$
\iota(x) \leq \iota(y)
$$

for all $x \mid W_{0}^{(2)}$ and $y \mid \prod_{\nu=m}^{2 m-2} W_{\nu}$.

Let $y_{0} \mid W_{2 m-2}$ with $\pi_{2}\left(\psi\left(y_{0}\right)\right) \neq q_{2}$ (such a $y_{0}$ exists, as discussed above, else Claim $\mathrm{C}$ follows). Let $W^{\prime}$ be an arbitrary product decomposition resulting from pulling up $y_{0}$ into a new product decomposition. Since $\pi_{2}\left(\psi\left(y_{0}\right)\right) \neq q_{2}$, we have (as discussed earlier) $\widetilde{\sigma}\left(W^{\prime}\right)=f_{1}^{m-1} f_{2}^{m-1}\left(f_{1}+f_{2}\right)$ with $\sigma\left(W_{0}^{\prime}\right)=f_{1}+f_{2}$. Let $X=\operatorname{gcd}\left(W_{0}^{(2)}, W_{0}^{\prime(2)}\right)$ and let $X^{\prime}, Y^{\prime}$ and $Y$ be defined by $W_{0}^{(2)}=X X^{\prime}, W_{0}^{\prime(2)}=X Y^{\prime}$ and $W_{2 m-2}=Y Y^{\prime}$. Thus $W_{2 m-2}^{\prime}=X^{\prime} Y$. Note that all four of these newly defined subsequences are nontrivial in view of $\sigma\left(\iota\left(W_{0}^{(2)}\right)\right) \equiv 1 \bmod n$ and $\sigma\left(\iota\left(W_{2 m-2}\right)\right) \equiv 0 \bmod n$.

Let $\mathcal{D}^{\prime}=\left\{W_{1}^{\prime}, \ldots, W_{m-1}^{\prime}\right\}$. In view of Lemma 3.24 and $\psi_{1}\left(n e_{1}\right) \neq 0$, it follows that we can apply Lemma 5.4 with $i=1, Z=W_{0}^{\prime(2)}$, and $\mathcal{D}$ taken to be $\mathcal{D}^{\prime}$ (however, do NOT change $I$ ). If 12 holds, then (in view of (13)) we can find $z \mid W_{j}^{\prime}$, for some $j \in[1, m-1]$, such that $\epsilon\left(y_{0}, z\right)=\epsilon(x, z)$, where $x \mid X$. Applying a type II swap between $z \mid W_{j}^{\prime}$ and each of $x \mid W_{0}^{\prime}$ and $y_{0} \mid W_{0}^{\prime}$, we conclude from Lemma 3.24 that $\psi_{1}(x)=\psi_{1}\left(y_{0}\right)$. However, since $x \mid X$ and $X \mid W_{0}^{(2)}$ and $y_{0} \mid W_{2 m-2}$, it follows from 35 that $\psi_{2}(x)=\psi_{2}\left(y_{0}\right)$ also, whence $\psi(x)=\psi\left(y_{0}\right)$, implying $q_{2}=\pi_{2}(\psi(x))=$ $\pi_{2}\left(\psi\left(y_{0}\right)\right)$, contrary to assumption. Therefore we may instead assume (11) holds. Moreover, if both $y_{0}$ and some $x \mid X$ are contained in the same interval $J_{i}$ (from (11)), then we can repeat the above argument to obtain the same contradiction. Therefore it follows, in view of (36), that $y_{0} \in J_{2}$ and $X \subset J_{1}$. 
Let $z \mid W_{0}^{\prime(2)}$ and $z^{\prime} \mid W_{j}^{\prime}$ with $j \geq m$ be arbitrary. Performing a type II swap between $z \mid W_{0}^{\prime(2)}$ and $z^{\prime} \mid W_{j}^{\prime}$, we conclude from Lemma $3.2,5$ that

$$
\psi_{2}(z)-\psi_{2}\left(z^{\prime}\right)+\psi_{2}\left(\epsilon\left(z, z^{\prime}\right) n e_{1}\right)=0 .
$$

Thus (35) implies that $\psi_{2}\left(\epsilon\left(z, z^{\prime}\right) n e_{1}\right)=0$, which, in view of $\psi_{2}\left(n e_{1}\right) \neq 0$ and (13), implies that $\epsilon\left(z, z^{\prime}\right)=0$ and

$$
\iota(z) \leq \iota\left(z^{\prime}\right)
$$

for any $z \mid W_{0}^{\prime(2)}$ and $z^{\prime} \mid W_{j}^{\prime}$ with $j \geq m$.

Applying (37) with $z \mid Y^{\prime}$ and $z^{\prime} \mid X^{\prime}$ and $j=2 m-2$, we conclude in view of (36) that

$$
\iota(z)=\max \left(\operatorname{supp}\left(\iota\left(W_{0}^{\prime(2)}\right)\right)\right)=\min \left(\operatorname{supp}\left(\iota\left(\prod_{\nu=m}^{2 m-2} W_{\nu}^{\prime}\right)\right)\right)=\iota\left(z^{\prime}\right)
$$

for any $z^{\prime} \mid X^{\prime}$ and $z \mid Y^{\prime}$.

From 38 applied with $z=y_{0}$, we see that there is $y_{0}^{\prime} \mid W_{0}^{(2)}$ with $\iota\left(y_{0}^{\prime}\right)$ $=\iota\left(y_{0}\right)$. Thus $y$ can be pulled up into a new decomposition $W^{\prime \prime}$ by exchanging $y_{0} \mid W_{2 m-2}$ and $y_{0}^{\prime} \mid W_{0}$, and all of the above arguments (valid for an arbitrary $W^{\prime}$ obtained by pulling up $y_{0} \mid W_{2 m-2}$ ) are applicable for $W^{\prime \prime}$. In particular, $y_{0}^{\prime-1} W_{0}^{(2)}=X \subset J_{1}$ and $y_{0} \in J_{2}$ imply, in view of $Y=y_{0}^{-1} W_{2 m-2}$, (11) and (38), that

$$
\max \left(\operatorname{supp}\left(\iota\left(y_{0}^{\prime-1} W_{0}^{(2)}\right)\right)\right)<\min \left(\operatorname{supp}\left(\iota\left(W_{2 m-2}\right)\right)\right) .
$$

If we could pull up $y_{0}^{\prime} y_{0} \mid W_{0} W_{2 m-2}$ into a new product decomposition $W^{\prime \prime \prime}$, then (39) would imply that $X^{\prime}$ contains a $z^{\prime}$ with $\iota\left(z^{\prime}\right)<\iota\left(y_{0}\right)$, which would contradict (37) applied with $z=y_{0}$ and $z^{\prime}=z^{\prime}$. Therefore we can assume otherwise, whence Theorem 2.6.2 and (39) imply that $\left|\operatorname{supp}\left(\iota\left(y_{0}^{\prime-1} W_{0}^{(2)}\right)\right)\right|=\left|\operatorname{supp}\left(\iota\left(y_{0}^{-1} W_{2 m-2}\right)\right)\right|=1$. Thus $\sigma\left(\iota\left(W_{0}^{(2)}\right)\right) \equiv 1$ $\bmod n$ and $\sigma\left(\iota\left(W_{2 m-2}\right)\right) \equiv 0 \bmod n$ force that $\iota\left(W_{2 m-2}\right)=g^{n}$ and $\iota\left(W_{0}^{(2)}\right)=$ $(g-1)^{n-1} g$, where $\iota\left(y_{0}\right)=\iota\left(y_{0}^{\prime}\right)=g$. Consequently, (11), $X \subset J_{1}$ and $y_{0} \in J_{2}$ (in the case when $W^{\prime}=W^{\prime \prime}$ ) force that $\iota(z)=g-1$ for all $z \mid y_{0}^{\prime-1} W_{0}^{(2)} \prod_{\nu=1}^{m-1} W_{i}$.

Applying type III swaps among the $W_{i}, i \in[1, m-1]$, we conclude from Lemma 3.3 .1 or 3.1.1 that $\psi(x)=q$ (say) for all $x \mid W_{i}, i \in[1, m-1]$. Applying type III swaps between $W_{0}$ and $W_{1}$, we conclude from Lemma 3.2 .3 or 3.1 .2 and Lemma 3.4 .3 that $\psi(x)=q$ for all $x \mid y_{0}^{\prime \prime-1} y_{0}^{\prime-1} W_{0}^{(2)}$, for some $y_{0}^{\prime \prime} \mid y_{0}^{\prime-1} W_{0}^{(2)}$, and that $\psi\left(y_{0}^{\prime \prime}\right)=q$ or $q+(C-1) f_{1}+f_{2}$. Applying a type III swap between $y_{0}^{\prime \prime} \mid W_{0}^{\prime \prime}$ and some $z \mid W_{1}^{\prime \prime}$ in $W^{\prime \prime}$, we conclude from Lemma 3.2. 4 that $\psi_{1}\left(y_{0}^{\prime \prime}\right)=\psi_{1}(z)=\psi_{1}(q)$, whence we see that $\psi\left(y_{0}^{\prime \prime}\right)=q+(C-1) f_{1}+f_{2}$ is impossible (since $C \equiv 1 \bmod m$ would con- 
tradict $W_{0} \notin \mathcal{C}_{0}$ when $W \in \Omega_{0}^{n u}$; see (31)). Thus $\psi\left(y_{0}^{\prime \prime}\right)=q$ as well, and $(g-1) e_{1}+e_{2}+q$ has multiplicity at least $m n-1$ in $S$, as desired, completing Case 3 .

CASE 4: $\Omega_{0}^{u}=\emptyset$ and $W_{0} \in \mathcal{C}_{0}$. We start with the following assertion.

A2. If $\Omega_{0}^{u}=\emptyset, W \in \Omega_{0}^{n u}$ with $\widetilde{\sigma}(W)=f_{1}^{m-1} f_{2}^{m-1}\left(f_{1}+f_{2}\right), W_{0} \in \mathcal{C}_{0}$, and $\left|\mathcal{A}_{2} \cap \mathcal{C}_{i}\right| \geq 1$ for all $i \in\{1,2\}$, then $I$ can be chosen such that one of the following properties holds:

(i) $\left|\operatorname{supp}\left(\psi\left(W_{0}^{(2)}\right)\right)\right|=1$, or

(ii) (a) $\psi_{i}\left(n e_{1}\right) \neq 0$ for all $i \in\{1,2\}$,

(b) there exist $g_{1}, g_{2} \in \mathbb{Z}$ such that $\operatorname{gcd}\left(g_{1}-g_{2}, n\right)=1$ and $\iota(U)=g_{1}^{n}$ and $\iota(V)=g_{2}^{n}$ for every $U \in \mathcal{A}_{2}^{*} \cap \mathcal{C}_{1}$ and $V \in \mathcal{A}_{2}^{*} \cap \mathcal{C}_{2}$,

(c) $g_{1}>g_{2}$ and $\iota(x) \leq g_{1}$ for all $x \mid W_{0}^{(2)}$,

(d) if also $\left|\mathcal{A}_{2} \cap \mathcal{C}_{i}\right| \geq 2$ for all $i \in\{1,2\}$, then there exist $c, d \in \operatorname{Ker}(\varphi)$ such that $\psi(U)=c^{n}$ and $\psi(V)=d^{n}$ for every $U \in \mathcal{A}_{2}^{*} \cap \mathcal{C}_{1}$ and $V \in \mathcal{A}_{2}^{*} \cap \mathcal{C}_{2}$.

Proof of A2. We may w.l.o.g. assume $\mathcal{C}_{1}$ are those blocks with sum $f_{1}$. Performing type II swaps between each $x \mid W_{0}^{(2)}$ and each $y \mid U \in \mathcal{A}_{2}^{*} \cap \mathcal{C}_{1}$, and between each $x \mid W_{0}^{(2)}$ and each $z \mid V \in \mathcal{A}_{2}^{*} \cap \mathcal{C}_{2}$, we conclude from Lemma 3.2 that

$$
\begin{aligned}
& \psi_{1}(x)=\psi_{1}(y)-\psi_{1}\left(\epsilon(x, y) n e_{1}\right), \\
& \psi_{2}(x)=\psi_{2}(z)-\psi_{2}\left(\epsilon(x, z) n e_{1}\right) .
\end{aligned}
$$

Since $\operatorname{ord}\left(e_{1}\right)=m n$, one of $\psi_{1}\left(n e_{1}\right)$ or $\psi_{2}\left(n e_{1}\right)$ is nonzero, say the former (the other case is identical). Then, in view of (40), we may apply Lemma 5.4 with $i=1, Z=W_{0}^{(2)}$ and $\mathcal{D}=\mathcal{A}_{2}^{*} \cap \mathcal{C}_{1}$. Consequently, we can choose $I$ such that

$$
\iota(x) \leq \iota(y)
$$

for all $x \mid W_{0}^{(2)}$ and $y \mid U \in \mathcal{A}_{2}^{*} \cap \mathcal{C}_{1}$, and $\psi_{1}$ is constant on $W_{0}^{(2)}$. If $\psi_{2}\left(n e_{1}\right)$ is zero, then 41 implies that $\psi_{2}$ is also constant on $W_{0}^{(2)}$, whence (i) holds. Therefore we may assume otherwise, and (a) is established. Likewise, if there is some $z \mid V \in \mathcal{A}_{2}^{*} \cap \mathcal{C}_{2}$ with $\iota(z) \geq \max \left(\operatorname{supp}\left(\iota\left(W_{0}^{(2)}\right)\right)\right)$ or $\iota(z)<$ $\min \left(\operatorname{supp}\left(\iota\left(W_{0}^{(2)}\right)\right)\right)$, then (i) again holds (in view of 13 and 41$)$ ). So we may assume otherwise:

$$
\min \left(\operatorname{supp}\left(\iota\left(W_{0}^{(2)}\right)\right)\right) \leq \iota(z)<\max \left(\operatorname{supp}\left(\iota\left(W_{0}^{(2)}\right)\right)\right)
$$


for all $z \mid V \in \mathcal{A}_{2}^{*} \cap \mathcal{C}_{2}$. Consequently, it follows in view of 42 that both $\operatorname{supp}\left(\iota\left(\prod_{U \in \mathcal{A}_{2}^{*} \cap \mathcal{C}_{1}} U\right)\right)$ and $\operatorname{supp}\left(\iota\left(\prod_{V \in \mathcal{A}_{2}^{*} \cap \mathcal{C}_{2}} V\right)\right)$ are disjoint.

Suppose $|\operatorname{supp}(\iota(U))|>1$ or $|\operatorname{supp}(\iota(V))|>1$ for some $U \in \mathcal{A}_{2}^{*} \cap \mathcal{C}_{1}$ or $V \in \mathcal{A}_{2}^{*} \cap \mathcal{C}_{2}$. Then we may find $u_{0} \mid U$ and $v_{0} \mid V$ such that $\left|\operatorname{supp}\left(\iota\left(u_{0}^{-1} U\right)\right)\right|$ $>1$ or $\left|\operatorname{supp}\left(\iota\left(v_{0}^{-1} V\right)\right)\right|>1$, whence it follows, in view of Theorem 2.6.2 (applied to $\iota\left(u_{0}^{-1} v_{0}^{-1} U V\right)$ modulo $\left.n\right)$ and the fact that $\operatorname{supp}\left(\iota\left(\prod_{U \in \mathcal{A}_{2}^{*} \cap \mathcal{C}_{1}} U\right)\right)$ and $\operatorname{supp}\left(\iota\left(\prod_{V \in \mathcal{A}_{2}^{*} \cap \mathcal{C}_{2}} V\right)\right)$ are disjoint, that we can refactor $U V=U^{\prime} V^{\prime}$ so that $U^{\prime}$ and $V^{\prime}$ both contain terms from both $U$ and $V$. Replacing the blocks $U$ and $V$ by $U^{\prime}$ and $V^{\prime}$ yields a new product decomposition $W^{\prime} \in \Omega_{0}$; in view of Lemma 3.2 , we still have $\widetilde{\sigma}\left(W^{\prime}\right)=\widetilde{\sigma}(W)$, whence $W^{\prime}$ satisfies the hypotheses of A2. However, since both $U^{\prime}$ and $V^{\prime}$ contain terms from both $U$ and $V$, it follows that both $U^{\prime}$ and $V^{\prime}$ contain a term $z^{\prime} \mid U$ with $\iota\left(z^{\prime}\right) \geq \max \left(\operatorname{supp}\left(\iota\left(W_{0}^{(2)}\right)\right)\right)$ (in view of 42$)$ ), as well as a term $z \mid V$ with $\min \left(\operatorname{supp}\left(\iota\left(W_{0}^{(2)}\right)\right)\right) \leq \iota\left(z^{\prime}\right)<\max \left(\operatorname{supp}\left(\iota\left(W_{0}^{(2)}\right)\right)\right)$ (in view of 433$)$ ), which makes it impossible for (11) or (12) to hold for $W^{\prime}$, contradicting Lemma 5.4 for $W^{\prime}$, which must hold by the above arguments. So we may assume $|\operatorname{supp}(\iota(U))|=1$ and $|\operatorname{supp}(\iota(V))|=1$ for all $U \in \mathcal{A}_{2}^{*} \cap \mathcal{C}_{1}$ and $V \in \mathcal{A}_{2}^{*} \cap \mathcal{C}_{2}$. Moreover, this argument also shows that if $\iota(U)=g_{1}^{n}$ and $\iota(V)=g_{2}^{n}$, then $\operatorname{gcd}\left(g_{1}-g_{2}, n\right)=1$.

Suppose $\left|\operatorname{supp}\left(\iota\left(\prod_{U \in \mathcal{A}_{2}^{*} \cap \mathcal{C}_{1}} U\right)\right)\right|>1$ or $\left|\operatorname{supp}\left(\iota\left(\prod_{V \in \mathcal{A}_{2}^{*} \cap \mathcal{C}_{2}} V\right)\right)\right|>1$, say the former (the other case will be identical). Then there are $U_{1}, U_{2} \in \mathcal{A}_{2}^{*} \cap \mathcal{C}_{1}$ and $V \in \mathcal{A}_{2}^{*} \cap \mathcal{C}_{2}$ with $\iota\left(U_{1}\right)=g_{1}, \iota\left(U_{2}\right)=g_{1}^{\prime}$ and $\iota(V)=g_{2}$, where $g_{1} \neq g_{1}^{\prime}$. We have $\operatorname{gcd}\left(g_{1}-g_{1}^{\prime}, n\right)=1$, else repeating the arguments of the previous paragraph, using $U_{1}$ and $U_{2}$ in place of $U$ and $V$, we obtain a $W^{\prime} \in \Omega_{0}$ satisfying the hypotheses of $\mathbf{A 2}$ but such that the conclusion of the previous paragraph fails, whence $1=\left|\operatorname{supp}\left(\psi\left(W_{0}^{\prime(2)}\right)\right)\right|=$ $\left|\operatorname{supp}\left(\psi\left(W_{0}^{(2)}\right)\right)\right|$ must hold by prior arguments, yielding (i). Hence, since $\operatorname{gcd}\left(g_{1}-g_{2}, n\right)=1$ and $\operatorname{gcd}\left(g_{1}^{\prime}-g_{2}, n\right)=1$, it follows that all $n$-term zero-sum modulo $n$ subsequences of $g_{1}^{n-1} g_{1}^{\prime n-1} g_{2}^{n-1}$ have support of cardinality three. Thus, by two applications of Theorem 2.6.1, we can refactor $U_{1} U_{2} V=X Y Z$ such that $X, Y$ and $Z$ all contain terms from each of $U_{1}$, $U_{2}$ and $V$ (note, since $|\operatorname{supp}(\iota(X))|=3$, that $\iota(Y Z) \subset g_{1}^{n-1} g_{1}^{\prime n-1} g_{2}^{n-1}$ ). Replacing $U_{1}, U_{2}$ and $V$ by $X, Y$ and $Z$ yields a new product decomposition $W^{\prime} \in \Omega_{0}$; in view of $\Omega_{0}^{u}=\emptyset$ and $m \geq 5$, we still have $\widetilde{\sigma}\left(W^{\prime}\right)=$ $\tilde{\sigma}(W)$, whence $W^{\prime}$ satisfies the hypotheses of A2. However, since $X, Y$ and $Z$ each contain terms from $U_{1}, U_{2}$ and $V$, we see that the condition $|\operatorname{supp}(\iota(U))|=1$ for $U \in \mathcal{A}_{2}^{*} \cap \mathcal{C}_{1}$ fails for $W^{\prime}$, whence previous arguments show $\left|\operatorname{supp}\left(\psi\left(W_{0}^{(2)}\right)\right)\right|=\left|\operatorname{supp}\left(\psi\left(W_{0}^{\prime(2)}\right)\right)\right|=1$, yielding (i). So we may assume $\left|\operatorname{supp}\left(\iota\left(\prod_{U \in \mathcal{A}_{2}^{*} \cap \mathcal{C}_{1}} U\right)\right)\right|=1$ and $\left|\operatorname{supp}\left(\iota\left(\prod_{V \in \mathcal{A}_{2}^{*} \cap \mathcal{C}_{2}} V\right)\right)\right|=1$, and also $\operatorname{supp}\left(\iota\left(\prod_{U \in \mathcal{A}_{2}^{*} \cap \mathcal{C}_{1}} U\right)\right)=g_{1}$ and $\operatorname{supp}\left(\iota\left(\prod_{V \in \mathcal{A}_{2}^{*} \cap \mathcal{C}_{2}} V\right)\right)=g_{2}$. This 
establishes (b). Moreover, by the arguments from the second paragraph, we can choose $I$ such that (c) holds.

We now assume $\left|\mathcal{A}_{2} \cap \mathcal{C}_{i}\right| \geq 2$ for all $i \in\{1,2\}$. Performing type III swaps between distinct $U_{1}, U_{2} \in \mathcal{A}_{2}^{*} \cap \mathcal{C}_{1}$ and between distinct $V_{1}, V_{2} \in \mathcal{A}_{2}^{*} \cap \mathcal{C}_{2}$, we conclude from Lemma 3.3 that $\psi(U)=c^{n}$ (say) for all $U \in \mathcal{A}_{2}^{*} \cap \mathcal{C}_{1}$ and that $\psi(V)=d^{n}$ (say) for all $V \in \mathcal{A}_{2}^{*} \cap \mathcal{C}_{2}$, establishing (d), and completing the proof of $\mathbf{A 2}$.

Since $\Omega_{0}^{u}=\emptyset$, it follows, in view of Lemma 3.3 , that if we pull up any term $y \mid U$, where $U \in \mathcal{A}_{2}^{*}$, then we may assume the resulting product decomposition still satisfies the hypothesis of Case 4 with $\widetilde{\sigma}\left(W^{\prime}\right)=\widetilde{\sigma}(W)$, else applying Case 3 using this product decomposition completes Claim C. Thus, if for every product decomposition satisfying the hypothesis of Case 4 we can find $I$ such that $\left|\operatorname{supp}\left(\psi\left(W_{0}^{(2)}\right)\right)\right|=1$, then, since modifying $I$ does not alter the values $\pi_{2}(\psi(x))$, we would be able to conclude $\left|\operatorname{supp}\left(\pi_{2}\left(\psi\left(S_{2}\right)\right)\right)\right|=1$ - by successively pulling up terms $y \mid S_{2}$, yielding a sequence of product decompositions satisfying the hypotheses of Case 4, until every such $y$ occurred in the $W_{0}^{(2)}$ part of one of these product decompositions, and then noting that there must always be a common term in $W_{0}^{(2)}$ between any two consecutive product decompositions in the sequence (in view of $\sigma\left(\iota\left(W_{0}^{(2)}\right)\right) \equiv 1 \bmod n$ ) completing Claim $\mathrm{C}$. Therefore we may assume this is not the case for $W$. Let w.l.o.g. $\widetilde{\sigma}(W)=f_{1}^{m-1} f_{2}^{m-1}\left(f_{1}+f_{2}\right)$ and $\mathcal{C}_{1}$ consist of those blocks with sum $f_{1}$.

Note that we must have $\mathcal{A}_{2}^{*} \cap \mathcal{C}_{1}$ and $\mathcal{A}_{2}^{*} \cap \mathcal{C}_{2}$ both nonempty, else in view of Claim B it would follow that $e_{1}$ is a term of $S$ with multiplicity $m n-1$, completing the proof. Thus A2(ii)(a) implies that $\psi_{i}\left(n e_{1}\right) \neq 0$ for $i \in\{1,2\}$. As a result, we cannot have a block $U \in \mathcal{A}_{1}^{*}$ (else $n e_{1}=\sigma(U)=f_{1}$ or $f_{2}$ ). Hence $\left|\mathcal{A}_{1}\right|=1$, implying $\left|\mathcal{A}_{2}^{*} \cap \mathcal{C}_{1}\right| \geq 2$ and $\left|\mathcal{A}_{2}^{*} \cap \mathcal{C}_{2}\right| \geq 2$. Thus, by choosing $I$ appropriately, $\mathbf{A 2}(\mathrm{ii})(\mathrm{a}-\mathrm{d})$ holds for $W$.

Suppose $\operatorname{supp}\left(\iota\left(W_{0}^{(2)}\right)\right) \neq\left\{g_{1}, g_{2}\right\}$. Then there must be some $x_{0} \mid W_{0}^{(2)}$ with $\iota\left(x_{0}\right) \notin\left\{g_{1}, g_{2}\right\}$ (in view of $\sigma\left(\iota\left(W_{0}^{(2)}\right)\right) \equiv 1 \bmod n$ ). Since $\operatorname{gcd}\left(g_{1}-\right.$ $\left.g_{2}, n\right)=1$, there is no $n$-term zero-sum $\bmod n$ subsequence of $g_{1}^{n-1} g_{2}^{n-1}$. Thus applying Theorem 2.6. 1 to $g_{1}^{n-1} g_{2}^{n-1} \iota\left(x_{0}\right)$ implies that we may find a subsequence $U_{1} \mid x_{0} U V$, where $U \in \mathcal{A}_{2}^{*} \cap \mathcal{C}_{1}$ and $V \in \mathcal{A}_{2}^{*} \cap \mathcal{C}_{2}$, such that $x_{0} \mid U_{1}$ and $\operatorname{supp}\left(\iota\left(x_{0}{ }^{-1} U_{1}\right)\right)=\left\{g_{1}, g_{2}\right\}$. Consequently, $\vee_{g_{i}}\left(U_{1}\right) \leq n-2$, and thus $\vee_{g_{i}}\left(\iota\left(U_{1}^{-1} W_{0}^{(2)} U V\right)\right) \geq 2$, for $i=\{1,2\}$. Thus, if there were no $n$-term zerosum $\bmod n$ subsequence of $\iota\left(U_{1}^{-1} u_{1}^{-1} v_{1}^{-1} W_{0}^{(2)} U V\right)$, where $u_{1}\left|U_{1}, v_{1}\right| V$ and $u_{1} v_{1} \mid U_{1}^{-1} V_{1}^{-1} U V$, then Theorem 2.6 2 would imply that $\iota\left(U_{1}^{-1} W_{0}^{(2)} U V\right)=$ $g_{1}^{n} g_{2}^{n}$, whence

$$
1 \equiv \sigma\left(\iota\left(W_{0}^{(2)} U V\right)\right) \equiv \sigma\left(\iota\left(U_{1}\right)\right)+n g_{1}+n g_{2} \equiv 0 \bmod n,
$$


which is a contradiction. Therefore we may assume there exists such a subsequence $\iota\left(U_{2}\right)$, where $U_{2} \mid U_{1}^{-1} u_{1}^{-1} v_{1}^{-1} W_{0}^{(2)} U V$. Let $W_{0}^{\prime}$ be defined by $W_{0} U V=U_{1} U_{2} W_{0}^{\prime}$. Then replacing $W_{0}, U$ and $V$ with $W_{0}^{\prime}, U_{1}$ and $U_{2}$ yields a new product decomposition $W^{\prime} \in \Omega_{0}$. Since $\Omega_{0}^{u}=\emptyset$ and $m \geq 4$, we must have $\tilde{\sigma}(W)=\tilde{\sigma}\left(W^{\prime}\right)$, and we may further assume $W_{0}^{\prime} \in \mathcal{C}_{0}$, else applying Case 3 using $W^{\prime}$ completes Claim C. Thus $W^{\prime}$ satisfies the hypotheses of Case 4 , but since $\left|\operatorname{supp}\left(\iota\left(U_{1}\right)\right)\right|>1$, we see that $W^{\prime}$ does not satisfy A2(ii). Thus A2(i) implies that we must have $\left|\operatorname{supp}\left(\pi_{2}\left(\psi\left(W_{0}^{\prime(2)}\right)\right)\right)\right|=1$ (note we do not have $\left|\operatorname{supp}\left(\psi\left(W_{0}^{\prime(2)}\right)\right)\right|=1$ as we would need to change $I$ for this to hold); since $u_{1} v_{1} \mid W_{0}^{\prime}$ and $u_{1} \mid U$ and $v_{1} \mid V$, this implies that $\pi_{2}(c)=\pi_{2}\left(\psi\left(u_{1}\right)\right)=\pi_{2}\left(\psi\left(v_{1}\right)\right)=\pi_{2}(d)$.

Let $x \mid x_{0}^{-1} W_{0}^{(2)}$ be arbitrary. By Theorem 2.6. 1 , it follows that there is an $n$-term zero-sum mod $n$ subsequence of $\iota\left(x^{-1} U_{1}^{-1} W_{0}^{(2)} U V\right)$, say $\iota\left(U_{3}\right)$ with $U_{3} \mid x^{-1} U_{1}^{-1} W_{0}^{(2)} U V$ (recall that $U_{1} \mid x_{0} U V$ ). Let $W_{0}^{\prime \prime}$ be defined by $W_{0} U V=U_{1} U_{3} W_{0}^{\prime \prime}$. Then replacing the blocks $W_{0}, U$ and $V$ with the blocks $W_{0}^{\prime \prime}, U_{1}$, and $U_{3}$ yields a new product decomposition $W^{\prime \prime} \in \Omega_{0}$, and as before we may assume $W^{\prime \prime}$ satisfies the hypotheses of Case 4 with $\widetilde{\sigma}\left(W^{\prime \prime}\right)=\widetilde{\sigma}(W)$. Thus, since $\left|\operatorname{supp}\left(\iota\left(U_{1}\right)\right)\right|>1$, we see that $W^{\prime \prime}$ does not satisfy A2(ii), and so we must have

$$
\left|\operatorname{supp}\left(\pi_{2}\left(\psi\left(W_{0}^{\prime \prime}(2)\right)\right)\right)\right|=1 .
$$

Since $x_{0} \mid U_{1}$ and $x_{0} \mid W_{0}^{(2)}$, it follows from the pigeonhole principle that we must have a term $x^{\prime} \mid W_{0}^{\prime \prime(2)}$ with $x^{\prime} \mid U V$, and thus with $\pi_{2}\left(\psi\left(x^{\prime}\right)\right)=$ $\pi_{2}(c)=\pi_{2}(d)$ (in view of the previous paragraph). Since $x \mid W_{0}^{\prime \prime}$, this implies $\pi_{2}(\psi(x))=\pi_{2}(c)$ (in view of (44)). As $x \mid x_{0}^{-1} W_{0}^{(2)}$ was arbitrary, we conclude that every $x \mid x_{0}^{-1} S_{2}$ has $\pi_{2}(\psi(x))=\pi_{2}(c)=\pi_{2}(d)$, completing the proof of Claim C (in view of A2(ii)(d) holding for $W$ ). So we may instead assume $\operatorname{supp}\left(\iota\left(W_{0}^{(2)}\right)\right)=\left\{g_{1}, g_{2}\right\}$.

Since $\left|\mathcal{A}_{1}\right|=1$, let $W_{1}, \ldots, W_{m-1}$ be the blocks of $\mathcal{A}_{2}^{*} \cap \mathcal{C}_{1}$, and let $W_{m}, \ldots, W_{2 m-2}$ be the blocks of $\mathcal{A}_{2}^{*} \cap \mathcal{C}_{2}$. Let $W_{0}^{(2)}=b_{1} \cdot \ldots \cdot b_{t} b_{1}^{\prime} \cdot \ldots \cdot b_{n-t}^{\prime}$ with $\iota\left(b_{i}\right)=g_{1}$ and $\iota\left(b_{j}^{\prime}\right)=g_{2}$. Applying type III swaps between $b_{i} \mid W_{0}$ and $y \mid W_{1}$, it follows from Lemma 3.34 that we may assume $\psi\left(b_{i}\right)=\psi(y)=c$ for all $i$ (else Case 3 completes Claim C). Likewise applying type III swaps between $b_{i}^{\prime} \mid W_{0}$ and $z \mid W_{m}$, it follows that $\psi\left(b_{i}^{\prime}\right)=\psi(z)=d$ for all $i$. Consequently, we may assume $t \in[2, n-2]$, else $S$ contains a term with multiplicity at least $m n-1$, as desired (either $g_{1} e_{1}+e_{2}+c$ or $g_{2} e_{1}+e_{2}+d$ ).

Applying type II swaps between $b_{1} \mid W_{0}$ and $z \mid W_{m}$ and between $b_{1}^{\prime} \mid W_{0}$ and $y \mid W_{1}$, it follows, in view of Lemma 3.2, (13) and $g_{1}>g_{2}(\mathbf{A 2} 2(\mathrm{ii})(\mathrm{c}))$, 
that

$$
\begin{array}{r}
d-c \in\left\langle f_{2}\right\rangle, \\
c-d+n e_{1} \in\left\langle f_{1}\right\rangle .
\end{array}
$$

Since $t \in[2, n-2]$, we have $b_{1} b_{2} \mid W_{0}^{(2)}$ and $b_{1}^{\prime} b_{2}^{\prime} \mid W_{0}^{(2)}$. Let $Y$ be a subsequence of $W_{1}$ and $Z$ be a subsequence of $W_{m}$ with $|Y|=|Z|=2$. Applying type II swaps between $b_{1}^{\prime} b_{2}^{\prime} \mid W_{0}$ and $Y \mid W_{1}$ and between $b_{1} b_{2} \mid W_{0}$ and $Z \mid W_{m}$, we conclude from Lemma 3.2 that

$$
\begin{gathered}
2(d-c)+\epsilon\left(b_{1}^{\prime} b_{2}^{\prime}, Y\right) n e_{1} \in\left\langle f_{2}\right\rangle, \\
2(c-d)+\epsilon\left(b_{1} b_{2}, Z\right) n e_{1} \in\left\langle f_{1}\right\rangle .
\end{gathered}
$$

Observe (in view of $g_{1}>g_{2}$ ) that

$$
\epsilon\left(b_{1}^{\prime} b_{2}^{\prime}, Y\right) n e_{1}= \begin{cases}0 & \text { if } g_{1}-g_{2} \leq(n-1) / 2 \\ -n e_{1} & \text { if } g_{1}-g_{2} \geq(n+1) / 2 .\end{cases}
$$

Likewise

$$
\epsilon\left(b_{1} b_{2}, Z\right) n e_{1}= \begin{cases}n e_{1} & \text { if } g_{1}-g_{2} \leq(n-1) / 2, \\ 2 n e_{1} & \text { if } g_{1}-g_{2} \geq(n+1) / 2 .\end{cases}
$$

Thus, if $g_{1}-g_{2} \leq(n-1) / 2$, then (48) and (46) imply that $c-d \in\left\langle f_{1}\right\rangle$, which combined with (45) implies that $c=d$, in which case Claim $\mathrm{C}$ follows. On the other hand, if $g_{1}-g_{2} \geq(n+1) / 2$, then (47) and 45) imply that $n e_{1} \in\left\langle f_{2}\right\rangle$, which contradicts $\mathbf{A 2}(\mathrm{ii})(\mathrm{a})$ for $W$, completing Case 4 .

Claim D. $\mathrm{h}(S)=m n-1$.

Proof. Let $S_{2}^{\prime}=x_{0}^{-1} S_{2}$, with $x_{0}$ as in Claim C, and let $S^{\prime}=S_{1} S_{2}^{\prime}$. By Proposition 4.2 and Claim B, we have $S_{1}=e_{1}^{\left|S_{1}\right|},\left|S_{1}\right|=\ell n-1$ and $\left|S_{2}^{\prime}\right|=$ $2 m n-\ell n-1$, for some $\ell \geq 1$. If $\ell \geq m$, then $e_{1}$ is a term with multiplicity at least $m n-1$, as desired. Therefore we may assume $\ell<m$. Moreover, since $S \in \mathcal{A}(G)$, it follows that $0 \notin \Sigma\left(S^{\prime}\right)$. In view of Claim C and Proposition 4.2. we may assume every $x_{i} \mid S_{2}^{\prime}$ is of the form $y_{i} e_{1}+(1+n q) e_{2}$, with $q \in[0, m-1]$. Let $T=\pi_{1}\left(S_{2}^{\prime}\right) \in \mathcal{F}\left(\left\langle e_{1}\right\rangle\right)$, and let $H^{\prime}=\left\langle e_{1},(1+q n) e_{2}\right\rangle \cong C_{m n} \oplus C_{r n}$, where $r n=\operatorname{ord}\left((1+q n) e_{2}\right)$. If $r<m$, then noting that $S^{\prime} \in \mathcal{F}\left(H^{\prime}\right)$ with $\left|S^{\prime}\right|=2 m n-2 \geq m n+r n-1=\mathrm{D}\left(H^{\prime}\right)$, we see that $0 \in \Sigma\left(S^{\prime}\right)$, contradicting $S \in \mathcal{A}(G)$. Thus we may choose $e_{2}$ to be $(1+q n) e_{2}$ while $\left(e_{1}, e_{2}\right)$ is still a basis, and so w.l.o.g. we assume $q=0$.

Since $\ell<m$, it follows that $\left|S_{2}^{\prime}\right|=2 m n-\ell n-1 \geq m n+n-1 \geq m n+2$ and

$$
\Sigma\left(S_{1}\right)=\left\{e_{1}, 2 e_{1}, \ldots,(\ell n-1) e_{1}\right\} .
$$

Consequently, $0 \notin \Sigma\left(S^{\prime}\right)$ implies

$$
\Sigma_{m n}\left(S_{2}^{\prime}\right)=\Sigma_{m n}(T) \subset A:=\left\{e_{1}, 2 e_{1}, \ldots,(m n-\ell n) e_{1}\right\},
$$


and thus

$$
\left|\Sigma_{m n}(T)\right| \leq m n-\ell n=|T|-m n+1 .
$$

Note that $\mathrm{h}(T)=\mathrm{h}\left(S_{2}^{\prime}\right) \leq m n-2$, else the proof is complete. Thus we can apply Theorem 2.7, taking $k=3$, whence it follows, in view of (51) and $0 \notin \Sigma_{m n}(T)$, that $|\operatorname{supp}(T)| \leq 2$.

We may assume $|\operatorname{supp}(T)|=2$, else $S$ will contain a term with multiplicity $|T|=2 m n-\ell n-1 \geq m n+n-1$, contradicting $S \in \mathcal{A}(G)$. Thus $T=\left(g_{0} e_{1}\right)^{n_{1}}\left(\left(g_{0}+d\right) e_{1}\right)^{n_{2}}$ for some $g_{0}, d \in \mathbb{Z}$ with $d e_{1} \neq 0$. Since $\left(e_{1}, g_{0} e_{1}+e_{2}\right)$ is also a basis for $G$, by redefining $e_{2}$ to be $g_{0} e_{1}+e_{2}$ we may w.l.o.g. assume $g_{0}=0$. Thus

$$
\Sigma_{m n}(T)=B:=\left(m n-n_{1}\right) d e_{1}+\left\{0, d e_{1}, \ldots,(m n-\ell n-1) d e_{1}\right\},
$$

which is an arithmetic progression of difference $d e_{1}$ and length $m n-\ell n$ (in view of $\left.0 \notin \Sigma_{n m}(T)\right)$. In view of $(50)$, we have $B=A$ with

$$
2 \leq n \leq|A|=m n-\ell n \leq m n-n \leq m n-2 .
$$

Thus $d e_{1}= \pm e_{1}$ (as the difference of an arithmetic progression under the above assumptions is unique up to sign). Consequently, (50) and (52) imply that $n_{1}=n m-1$ if $d e_{1}=e_{1}$ (since $\left.\left|S^{\prime}\right| \leq 2 n m-2\right)$, and that $n_{1}=m n-\ell n$ if $d e_{1}=-e_{1}$ (since $\left.\left|S_{2}^{\prime}\right|<2 m n-\ell n\right)$. However, in the former case, $e_{2}$ has the desired multiplicity in $S$, while in the latter case, $n_{2}=2 m n-\ell n-1-n_{1}=$ $m n-1$, and thus $d e_{1}+e_{2}=-e_{1}+e_{2}$ has the desired multiplicity, completing the proof.

6. Proof of the Corollary. Let $G=C_{n_{1}} \oplus C_{n_{2}}$, with $1<n_{1} \mid n_{2}$, and suppose that, for every prime divisor $p$ of $n_{1}$, the group $C_{p} \oplus C_{p}$ has Property B. The assertion that $C_{n_{1}} \oplus C_{n_{1}}$ has Property $\mathbf{B}$ follows from the Theorem and from the following two statements:

(a) For every $n \in[2,10]$, the group $C_{n} \oplus C_{n}$ has Property B: for $n \leq 6$ this may be found in [9, Proposition 4.2]; the cases $n \in\{8,9,10\}$ (and more) are settled in [2].

(b) If $n \geq 6$ and $C_{n} \oplus C_{n}$ has Property $\mathbf{B}$, then $C_{2 n} \oplus C_{2 n}$ has Property $\mathbf{B}$ (see [9, Theorem 8.1]).

Since $C_{n_{1}} \oplus C_{n_{1}}$ has Property $\mathbf{B}$, the characterization of the minimal zerosum sequences over $G$ of length $\mathrm{D}(G)$ now follows from the main result in [21] (which differs from the Corollary only in that its hypothesis is that $C_{n_{1}} \oplus C_{n_{1}}$ has Property $\mathbf{B}$, rather than that $C_{p} \oplus C_{p}$ has Property $\mathbf{B}$ for every prime divisor $p$ of $n_{1}$ ).

Acknowledgments. This work was partially supported by NSFC with grant no. 10671101 and by the 973 Project with grant no $9732006 \mathrm{CB} 805904$. 
It was further supported by the Austrian Science Fund FWF (Project Number M1014-N13). We also wish to thank the referees for their suggestions for improving the manuscript.

Note added in proof. When this article went to press in December 2009, Christian Reiher announced a proof that $C_{p} \oplus C_{p}$ has Property B for all primes $p \in \mathbb{P}$. This implies that the assumption in the Corollary is satisfied, and thus the structure of all minimal zero-sum sequences of maximal length over groups of rank two is completely determined.

\section{References}

[1] G. Bhowmik, I. Halupczok, and J.-C. Schlage-Puchta, Inductive methods and zerosum free sequences, Integers 9 (2009), Paper A40, 515-536.

[2] - - - - - The structure of maximal zero-sum free sequences, Acta Arith., to appear.

[3] A. Bialostocki and P. Dierker, On the Erdös-Ginzburg-Ziv theorem and the Ramsey numbers for stars and matchings, Discrete Math. 110 (1992), 1-8.

[4] Y. Edel, Sequences in abelian groups $G$ of odd order without zero-sum subsequences of length $\exp (G)$, Des. Codes Cryptography 47 (2008), 125-134.

[5] Y. Edel, C. Elsholtz, A. Geroldinger, S. Kubertin, and L. Rackham, Zero-sum problems in finite abelian groups and affine caps, Quart. J. Math. Oxford 58 (2007), 159-186.

[6] C. Elsholtz, Lower bounds for multidimensional zero sums, Combinatorica 24 (2004), 351-358.

[7] W. D. Gao and A. Geroldinger, On long minimal zero sequences in finite abelian groups, Period. Math. Hungar. 38 (1999), 179-211.

[8] - - - On the order of elements in long minimal zero-sum sequences, ibid. 44 (2002), $63-73$.

[9] - - - On zero-sum sequences in $\mathbb{Z} / n \mathbb{Z} \oplus \mathbb{Z} / n \mathbb{Z}$, Integers 3 (2003), Paper A08, 45 pp.

[10] - - - Zero-sum problems in finite abelian groups: a survey, Expo. Math. 24 (2006), $337-369$.

[11] W. D. Gao, A. Geroldinger, and W. A. Schmid, Inverse zero-sum problems, Acta Arith. 128 (2007), 245-279.

[12] W. D. Gao, Q. H. Hou, W. A. Schmid, and R. Thangadurai, On short zero-sum subsequences II, Integers 7 (2007), Paper A21, 22 pp.

[13] A. Geroldinger, Additive group theory and non-unique factorizations, in: Combinatorial Number Theory and Additive Group Theory, A. Geroldinger and I. Ruzsa (eds.), Adv. Courses Math. CRM Barcelona, Birkhäuser, 2009, 1-86.

[14] A. Geroldinger and F. Halter-Koch, Non-Unique Factorizations. Algebraic, Combinatorial and Analytic Theory, Pure Appl. Math. 278, Chapman \& Hall/CRC, 2006.

[15] B. Girard, Inverse zero-sum problems in finite abelian p-groups, Colloq. Math., to appear.

[16] D. J. Grynkiewicz, On a conjecture of Hamidoune for subsequence sums, Integers 5 (2005), Paper A07, 11 pp.

[17] Y. ould Hamidoune, Subsequence sums, Combin. Probab. Comput. 12 (2003), 413425 .

[18] M. B. Nathanson, Additive Number Theory: Inverse Problems and the Geometry of Sumsets, Springer, 1996.

[19] S. Savchev and F. Chen, Minimal zero-sum sequences of maximum length in the group $C_{3} \oplus C_{3 k}$, Integers 7 (2007), Paper A42, 6 pp. 
[20] W. A. Schmid, The inverse problem associated to the Davenport constant for $C_{2} \oplus$ $C_{2} \oplus C_{2 n}$, and applications to the arithmetical characterization of class groups, submitted.

[21] - Inverse zero-sum problems II, to appear.

[22] W. A. Schmid and J. J. Zhuang, On short zero-sum subsequences over p-groups, Ars Combin., to appear.

Weidong Gao

Center for Combinatorics

Nankai University

Tianjin 300071, P.R. China

E-mail: wdgao_1963@yahoo.com.cn
Alfred Geroldinger, David J. Grynkiewicz Institut für Mathematik und Wissenschaftliches Rechnen Karl-Franzens-Universität Graz Heinrichstraße 36 8010 Graz, Austria E-mail: alfred.geroldinger@uni-graz.at diambri@hotmail.com

Received on 3.2.2008

and in revised form on 15.5.2009 